\title{
Exercise for the prevention and treatment of adolescent-onset nicotine addiction
}

\section{Victoria Sanchez}

Montgomery Village, MD

B.S., Frostburg State University, 2007

\author{
A Dissertation presented to the Graduate Faculty \\ of the University of Virginia in Candidacy for the Degree of \\ Doctor of Philosophy \\ Department of Neuroscience \\ University of Virginia \\ December, 2013
}

Wendy J. Lynch, PhD (Advisor)

Ignacio Provencio, $\mathrm{PhD}$ (Committee Chair)

Alev Erisir, MD, $\mathrm{PhD}$

Michael M. Scott, PhD

Jill Venton, $\mathrm{PhD}$ 


\begin{abstract}
Cigarette smoking is the leading cause of preventable death in the United States followed closely by obesity. Given that most adult smokers initiate tobacco use during adolescence and find it difficult to quit, preventative measures to curtail adolescent smoking initiation are necessary. Thus, one objective of this dissertation was to determine, using animal models, whether exercise might block the initiation of nicotine use. As the use of pharmacotherapies is controversial in teens due to ongoing neurodevelopment, another objective of this dissertation was to determine whether exercise would prevent nicotine relapse. Vulnerability to nicotine addiction is also apparent in women and adolescent females as they have been found to progress to dependence faster, have more difficulty quitting, a greater vulnerability to relapse, and to be at a higher risk for the smoking-related diseases than their male counterparts despite equal or even lower levels of use. Adolescent and adult female rodents also have a greater propensity for nicotine self-administration, suggesting that these sex differences in vulnerability to nicotine addiction are biological based. Thus, another objective of this dissertation was to determine if the efficacy of exercise as an intervention for nicotine addiction differs between males and females in an adolescent-onset model. Nicotine is known to produce long-lasting changes in the reward pathway, and changes within the nucleus accumbens in particular, are thought to underlie vulnerability to relapse. Given that the mechanisms for the efficacy of exercise are unknown, the final objective of this dissertation was to examine the possibility that its efficacy is related to its ability to modulate these nicotine-induced adaptations. To address these objectives, I examined the
\end{abstract}


efficacy of voluntary wheel running, an animal model of aerobic exercise, at reducing nicotine use initiation and relapse vulnerabilities in adolescent male and female rats. I used electron microscopy to examine the effect of exercise on nicotine-induced structural plasticity of synapses within the nucleus accumbens. I found that contemporaneous exposure to brief bouts of exercise prevented acquisition of nicotine self-administration in adolescent rats. Exposure to brief bouts of exercise during an abstinence period also significantly attenuated subsequent nicotine-seeking in both male and female rats. However, in contrast to males, females displayed an enhanced sensitivity to the effects of environmental enrichment and/or non-aerobic exercise in a locked running wheel control condition and showed attenuated nicotine-seeking under both locked and unlocked wheel conditions. I also showed that exercise during abstinence normalized nicotine abstinenceinduced structural plasticity of excitatory synapses in the nucleus accumbens. Together, the results from this dissertation demonstrate that exercise, by modulating plasticity within the reward pathway, may be an effective prevention and intervention for adolescent-onset nicotine addiction. 


\section{CREDITS}

Portions of this dissertation were published previously and are republished here with the permission of the publishing groups and co-authors. References for Chapters 3,4 , and the Appendix are listed below.

\section{Chapter 3}

Sanchez V, Moore CF, Brunzell DB, Lynch WJ (2013) Effect of wheel-running during abstinence on subsequent nicotine-seeking in rats. Psychopharmacology 227: 403-411.

\section{Chapter 4}

Sanchez V, Moore CF, Brunzell DB, Lynch WJ. Sex differences in the effect of exercise during an abstinence period on subsequent nicotine-seeking. Psychopharmacology. In press. DOI: $10.1007 / \mathrm{s} 00213-013-3359-3$

\section{Appendix}

Sanchez V, Feinstein SD, Lunardi N, Joksovic PM, Boscolo A, Todorovic SM, JevtovicTodorovic V (2011) General anesthesia causes long-term impairment of mitochondrial morphogenesis and synaptic transmission in developing rat brain. Anesthesiology. 115(5): 992-1002. 


\section{TABLE OF CONTENTS}

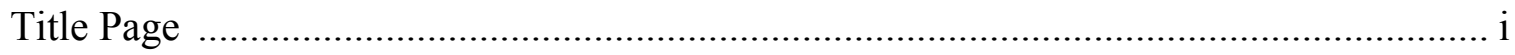

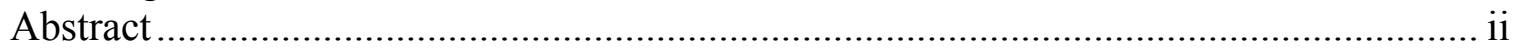

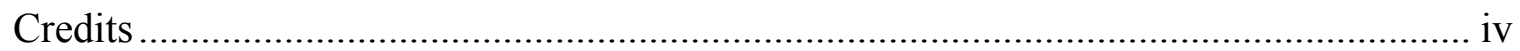

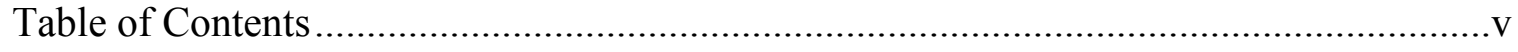

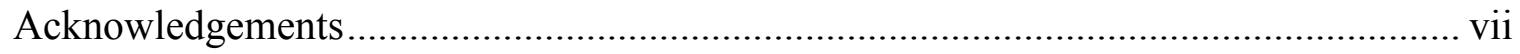

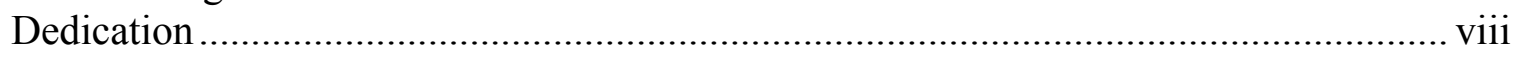

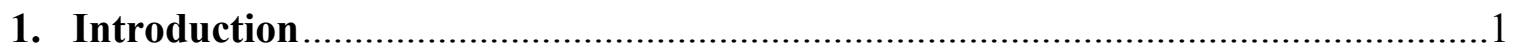

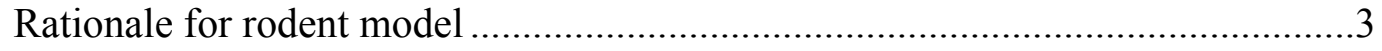

Adolescent vulnerability to nicotine addiction .............................................6

Female vulnerability to nicotine addiction ................................................... 7

Exercise as a prevention for nicotine addiction ..............................................

Exercise as an intervention for nicotine addiction..........................................11

Neurobiology of nicotine addiction ............................................................ 13

2. Wheel running exercise as a prevention for initiation of nicotine use .................19

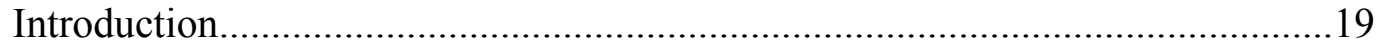

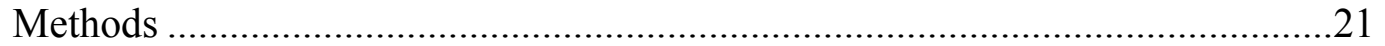

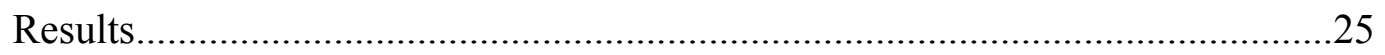

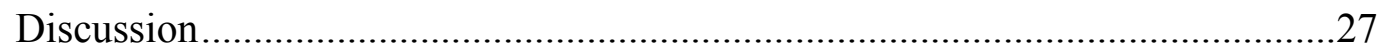

3. Wheel running during abstinence as an intervention for nicotine relapse...........30

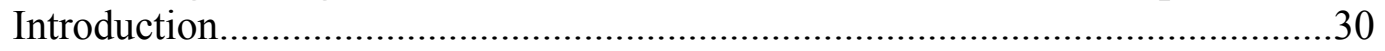

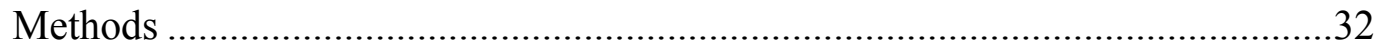

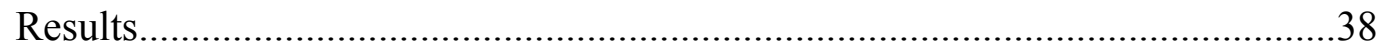

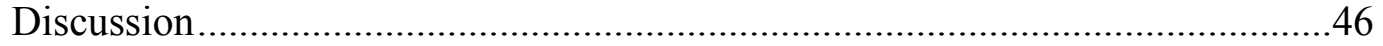

4. Sex differences in wheel running as an intervention for nicotine relapse ............50

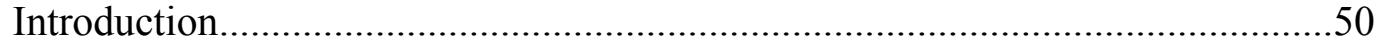

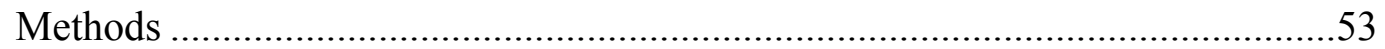

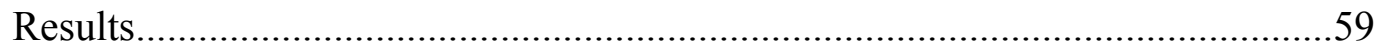

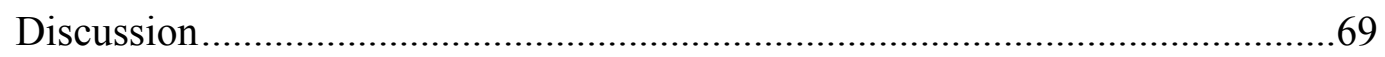

5. Structural plasticity associated with wheel running as an intervention .............75

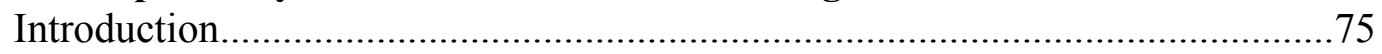

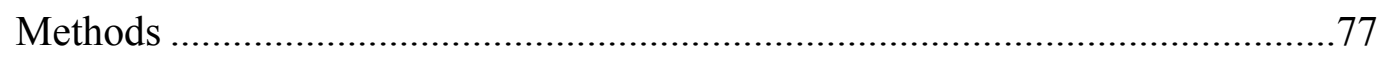

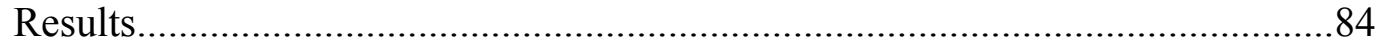

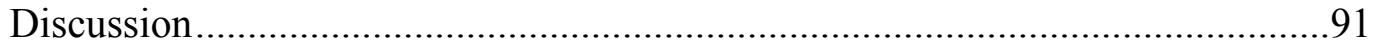




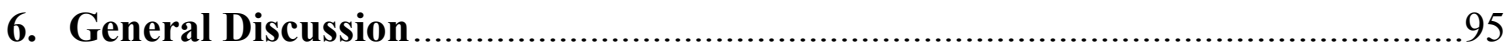

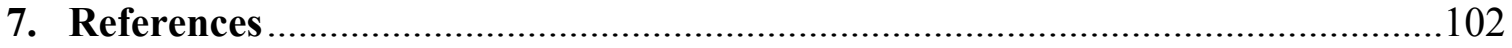

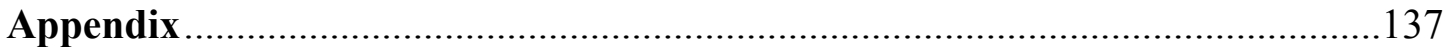




\section{ACKNOWLEDGEMENTS}

I would like to thank my mentor, Wendy Lynch, for her relentless support, encouragement, and confidence in me. I would also like to thank Iggy Provencio, Alev Erisir, Michael Scott, and Jill Venton, for their guidance and support along the way. I would like to give a special thanks to Sue Moenter and Tracy Mourton for their assistance in keeping the dream alive. I would also like to acknowledge Bettina Winckler, Manoj Patel, Nadia Cempré and the Neuroscience Graduate Program for their support and assistance. I would also like to thank Susie Doyle, Carolina Ramôa, and Alexis Peterson and incredible undergraduate assistants who helped me tremendously: Catherine Moore, Pallvi Chopra, Amanda Imbriglia, Andrea Chernau and Matthew Lycas. The McNair Scholars program of the University of Maryland deserves special recognition for helping me to achieve this degree. Last but not least, I would like to thank my incredible family and friends for the constant love and support. This work was supported by the Neurobiology and Development training grant (T32 HD007323), a National Research Service Award (F31 DA033087) from the National Institute on Drug Abuse, and grants from the Virginia Foundation for Healthy Youth (8520667 and 8520893). 


\section{DEDICATION}

For Ana Lydia Rios Medina 


\section{CHAPTER 1: \\ Introduction}

Smoking is the leading cause of preventable death in the United States and a major health concern worldwide, accounting for nearly 5 million deaths each year (World Health Organization 2009). People continue to smoke despite the risk of disease due to their developed addiction to nicotine, the primary addictive component of tobacco. Most smokers initiate use during adolescence. A steady decline in rates of smoking initiation over the last several decades indicates that prevention strategies have been somewhat effective (Substance Abuse and Mental Health Services Administration 2013). However, rates of smoking initiation are still quite high with approximately 700 people under the age of 18 initiating use per day (SAMHSA 2013), suggesting that the continued investigation of methods that could improve current prevention strategies or aid in the development of novel prevention strategies is necessary. Adolescents are believed to be particularly vulnerable to nicotine addiction following initiation because they display an accelerated progression to dependence and vulnerability to persistent heavy smoking in adulthood. Most of the currently available cessation aids for tobacco addiction replace nicotine or mimic its actions at the nicotinic acetylcholine receptors. However, the use of such pharmacotherapies is controversial for use in adolescents due to ongoing neurodevelopment and there are currently no FDA approved cessation aids for people under the age of 18 years old. Sex is another vulnerability factor with women displaying a more rapid progression to dependence and greater difficulty quitting as compared to men (Perkins et al. 1999). Therefore, the identification of novel sex-specific preventions 
as well as developmentally safe cessation strategies for adolescent males and females is necessary.

Physical activity, and specifically exercise, has been suggested as a potential nonpharmacological intervention and treatment for nicotine addiction that also has secondary health benefits (i.e., prevention of obesity and related diseases). This is an intriguing option based on promising initial findings and because it is presumably safe for use in adolescents and relatively inexpensive to implement. Clinical and preclinical evidence suggests that exercise may protect against both the initiation of drug use as well as relapse to drug use in abstainers. Epidemiological studies have demonstrated that physical activity in adolescents is associated with lower levels of smoking and other drug use, although direct causative effects are not yet known. Exercise during abstinence from smoking has been shown to acutely reduce cravings for cigarettes as well as alleviate psychological issues that are known to contribute to relapse in adults (Bock et al. 1999; Taylor and Katomeri 2007; Janse Van Rensburg et al. 2009, Haasova et al. 2012). Although, not yet examined for nicotine, preclinical studies using other drugs of abuse, such as cocaine, have demonstrated that wheel and treadmill running, animal models of aerobic exercise, can decrease initiation, use, and drug-seeking in adult male and female rats (see Lynch et al. 2013 for review). There also appears to be a sex differences in the effect of exercise at reducing cocaine self-administration where efficacy appears to be greater in females as compared to males (Cosgrove et al. 2002). Thus, exercise is an intriguing option for the prevention and treatment of adolescent-onset nicotine addiction in both males and females. 
The objectives of the research presented in this dissertation are to assess the potential of exercise to prevent the initiation of nicotine use, prevent relapse following abstinence, assess the effectiveness of exercise in females, and identify potential mechanisms by which exercise exerts its beneficial effects in adolescents. In this chapter, rationale for the use of a rodent model of exercise will be presented along with further clinical and preclinical support for adolescent and female vulnerability to nicotine addiction. The available preclinical and clinical data on the use of exercise as an addiction therapy will be reviewed. Lastly, the neurobiology underlying nicotine addiction is discussed and potential mechanisms by which exercise might prevent initiation and facilitate prolonged abstinence are proposed.

\section{Rationale for a rodent model}

Although our ultimate goal is to apply these studies to humans, it is difficult in human studies to control for psychosocial influences that motivate smoking behavior. Animal models of nicotine addiction, however, enable experimental control over environmental parameters. Animal studies are particularly valuable in this regard since it is not ethical to study the factors that influence the initiation or resumption of smoking in drug-naïve or drug-abstinent humans except by retrospective reports and long-term epidemiological studies. Initiation of drug use, or acquisition, is a transitional phase of

addiction when one goes from initial exposure to regular use. This phase is best modeled in rodents using a self-administration paradigm. During daily sessions animals perform 
an operant response to receive an infusion of drug and the amount of time needed to meet a certain level or stability of intake is a measure of acquisition vulnerability. Relapse vulnerability in assessed in rodents using an extinction/reinstatement paradigm. As in humans, many different types of cues can elicit drug-seeking in animals, including stress, the cues formerly associated with the drug, and priming injections of the drug itself (Shaham et al. 2003). Responding during extinction sessions, which are not reinforced by the drug, as well as the rate by which responding becomes extinguished, serve as measures of drug-seeking in the absence of cues. Reinstatement testing is then assessed under various conditions. For example, under a cue-induced paradigm like the one used in these studies, the cues formerly associated with the drug are presented at the beginning of the session and following lever-presses on the drug-associated lever. Total lever presses thus serve as a measure of drug-seeking in response to drug associated cues. These animal models of drug use initiation and relapse vulnerability can be useful for determining the biological basis for the efficacy of novel prevention and intervention strategies without the influence of psychosocial factors.

Several studies have examined the effects of exercise in these acquisition and reinstatement paradigms (e.g., Smith et al. 2011; 2012 Lynch et al. 2010; Zlebnik et al. 2010; Smith and Pitts 2011; Sanchez et al. 2013a; b; Peterson et al. 2013), and like results in humans the initial findings are promising. It is worth briefly discussing the many variations in schedules of exercise that have been used in this field. The first consideration is relationship of the exposure of exercise with respect to drug selfadministration which varies with the research question addressed. Most studies have 
analyzed the effect of exercise when available contemporaneously, but not concurrently to drug administration, for example, when the exercise condition is available in the home cage but not during drug self-administration session. This design allows for an examination of the effects of contemporaneously available exercise on ongoing drugtaking and seeking behavior. In contrast, other studies have made exercise available concurrently with drug self-administration to examine its effects as a competing nondrug reinforcer. Some studies have examined the effects of exercise prior to or after but not during the self-administration phase to assess potential lasting protective effects of exercise. Another factor is the type exercise made available to the animal whether it is forced exercise on a treadmill or voluntary in a running wheel. Treadmill running is advantageous over voluntary wheel running in that it allows for experimenter control over distance and intensity of exercise. However, this form of exercise can also be stressful which itself can affect drug-taking and seeking behaviors (Piazza and Moal 1998). Voluntary wheel running circumvents the issue of stress; however, the distance and intensity of exercise cannot be controlled and levels of running can be quite variable. The length of exercise exposure has also been manipulated with levels of access varying from 24-hours over a prolonged period of time to less than an hour during brief bouts. Notably, most studies that have used voluntary access to running wheels as a model of aerobic exercise have allowed unlimited access to running wheels. Little information is available on the effects of limited voluntary exercise, which may be a more appropriate model of human exercising behavior. 
Adolescent vulnerability to nicotine addiction

The acquisition of many drugs of abuse begins during adolescence and this is particularly true for nicotine. A recent survey of drug use in the United States (SAMHSA, 2013) revealed that an estimated 57.5 million Americans over the age of 12 are current smokers. When current use is compared across ages, an exponential increase is observed is observed between ages 12 and 20 and then these rates remain constant well into late adulthood. Furthermore, of the 2.3 million people who initiated smoking in the past year, $51.4 \%$ were under the age of 18 (SAMSHA 2013). Many studies have associated an early age of smoking onset with greater probability of being a heavy smoker and having a greater difficulty successfully quitting later in life (Breslau and Peterson 1996; Chen and Millar 1998; Taioli and Wynder 1991; Hu et al. 2006; Colby et al. 2000). Adolescents also appear to progress more rapidly to addiction after initial tobacco use and display greater levels of dependence despite lower levels of use as compared to adults (Tanski et al. 2004). Thus, it appears that adolescents are more vulnerable to initiating smoking and to succumbing to the addiction than adults.

Similar to humans, adolescent rodent models display increased vulnerability to nicotine addiction-related behaviors. Adolescents have been found to be more sensitive to the rewarding effects of nicotine as assessed under the conditioned place preference (CPP) procedure. In this procedure, which utilizes Pavlovian conditioning, nicotine and vehicle are administered in separate chambers with distinct tactile and sensory cues. In test sessions, animals are permitted to enter either chamber to determine their preference. In comparing adolescent versus adult rodents, adolescents have been found to prefer the 
nicotine paired chamber at lower nicotine doses than adults (Vastola et al. 2002; Kota et al. 2007; Torres et al. 2008; Torrella et al. 2004; Shram et al. 2006; 2010). Adolescents also display significant place preference after just one conditioning session while adults do not (Brielmaier et al. 2007) further underscoring their heightened vulnerability. Differences in the rewarding properties of nicotine have also been demonstrated during the adolescent period, with younger adolescent rats displaying increased sensitivity to nicotine as compared to older adolescents under CPP conditions (Belluzi et al. 2004). The use of self-administration paradigms, in which the animal learns to perform an operant response for the delivery of a drug infusion, have been used for many drugs of abuse including nicotine to study their reinforcing properties. Intravenous (IV) nicotine selfadministration is used to model the smoke inhalation route used by humans since delivery to the brain is rapid with this method. Several reports have demonstrated that adolescents will self-administer more IV nicotine infusions than adults (Levin 2003; 2007; 2011; Chen et al. 2007; but see Shram et al. 2007; 2008). Thus, as in humans, adolescent rats appear to more vulnerable to nicotine addiction as compared to adults.

Female vulnerability to nicotine addiction

Rates of smoking have historically been higher in males than females, which has led to the impression that men are more vulnerable to nicotine addiction than women. However, substantial evidence with smoking behavior, as well as with other drugs, indicates that females are more vulnerable to certain aspects of addiction than males. For example, although the historically higher rates of smoking in males than females are 
maintained in current data among adult populations (33\% versus $21 \%)$, such differences are absent among adolescent populations $(6.8 \%$ in males and $6.3 \%$ in females; SAMHSA 2013). Evidence of enhanced vulnerability in females is also apparent in studies demonstrating that both adult and adolescent females develop dependence to nicotine more rapidly after initial use as compared to males of their age (DiFranza et al. 2002; Ridenour et al. 2006; Thorner et al. 2007; Pierce and Gilpin 1996). It has also been reported that women are not as responsive as men to currently available cessation aids such as nicotine replacement therapies (Cepeda-Benito et al. 2004; Perkins and Scott 2008) and may be at a greater risk for relapse as compared to men (Japuntich et al. 2011; Piper et al. 2010). Furthermore, women are more vulnerable to tobacco carcinogens then men despite reporting smoking fewer cigarettes (Zang and Wynder 1996). Women also appear to be more vulnerable to myocardial infarction (Prescott et al. 1998) and coronary heart disease (Huxley and Woodward 2011) than men due to smoking. Thus, the development of smoking cessation therapies that are more effective in females is desperately needed.

Although few studies have examined sex differences in nicotine selfadministration, the findings available are consistent with the idea of an enhanced sensitivity in females (Donny et al. 2000, Chaudri et al. 2005; Lynch 2009; Li et al. 2012; Levin et al. 2011; Klein et al. 2004). Adult and adolescent females have been found to acquire nicotine self-administration more rapidly than males under low dose conditions, suggesting that females are more sensitive to the positive reinforcing effects of nicotine (Donny et al. 2000; Lynch 2009). Furthermore, females respond at higher levels under a 
progressive ratio, where response requirements increase with each subsequent infusion, and to display a shorter latency to first response, suggesting they are more motivated to self-administer nicotine (Donny et al. 2000; Lynch 2009; Chaudri et al. 2005; Li et al. 2012). Similarly, adult female rats have been found to consume more oral nicotine solution as compared to males (Klein et al. 2004). Thus, similar to humans, female rodents appear to be more vulnerable to nicotine addiction than males and as such, may require different prevent/intervention strategies.

\section{Exercise as a prevention for nicotine addiction}

Exercise is a promising non-pharmacological prevention for adolescent-onset nicotine addiction. A plethora of epidemiological data exists on the association of physical activity levels and drug use in adolescents collected from surveys, longitudinal studies, and twin studies. Cross-sectional studies examining data collected from in-school surveys consistently report that physically active teens are less likely to smoke cigarettes as compared to less physically active teens (e.g., Pastor et al. 2003; Escobedo et al. 1993; Field et al. 2001; Melnick et al. 2001; Naylor et al. 2001; Pate et al. 1996; 2000; Rainey et al. 1996; Mattila et al. 2012; Castrucci et al. 2004). Similarly, longitudinal studies following a sample population from adolescence to early adulthood report that lower levels of smoking over many years is associated with higher levels of initial and continued physical activity (Nelson and Gordon-Larsen 2006; Kujala et al. 2007; Wichstrøm and Wichstrøm 2009; Korhonen et al. 2009). Results from longitudinal studies in twins that differed in their physical activity levels at baseline show that a 
persistently inactive twin is more likely to smoke and use other drugs as compared to their more active twin (Kujala et al. 2007; Korhonen et al. 2009). However, a caveat to these epidemiological findings is that the measure of physical activity or exercise is often associated sports team participation. Thus, it is unclear whether the effect of increased physical activity on smoking behavior is due to a biological effect of exercise or to psychosocial influences of participating in a particular sport (e.g. expectations and influences of teammates, coaches, etc.). In partial support of the latter, some data indicate that participation in team sports may provide no protection or even increased risk of alcohol and smokeless tobacco use (Pate et al. 1996, 2000; Terry-McElrath and O'Malley 2011; Peretti-Watel et al. 2003; Martinsen and Sundgot-Borgen 2012). A direct causal effect of exercise in preventing initiation of drug use is difficult to determine with humans.

Animal studies support a biological basis for an effect of exercise in preventing drug use initiation. As mentioned above, the initiation of drug use is modeled in animals with a self-administration acquisition paradigm. Voluntary wheel running exercise prior to and contemporaneous with acquisition testing has been shown to reduce rates of cocaine and methamphetamine acquisition (Smith and Pitts 2011; Engelmann et al. 2013), suggesting that exercise can reduce vulnerability during the drug acquisition phase. This idea is further supported by studies utilizing CPP paradigms, which have demonstrated that prior exercise, either voluntarily on a running wheel or forced on a treadmill, prevents the development of a preference for an environment paired with MDMA, amphetamine or cocaine (Lett et al. 2002; Rozeske et al. 2011; El Rawas et al. 
2009; Solinas et al. 2008; Fontes-Ribeiro et al. 2011; Thanos et al. 2010). The effect of exercise has also been found to extend beyond initial vulnerability and to decrease intake during maintenance phases of drug self-administration. When voluntary wheel running is available concurrently with cocaine self-administration it has been shown to reduce intake and prevent escalation of use in adults and adolescents (Cosgrove et al. 2002; Zlebnik et al. 2012). Under contemporaneous conditions, exercise has been found to decrease heroin, morphine, methamphetamine, and cocaine self-administration (Smith and Pitts 2012; Hosseini et al. 2009; Engelmann et al. 2013; Smith and Witte 2012). This effect seems to be independent of whether an extensive exercise-training period occurred prior to self-administration. That these findings have been reported under both concurrent and contemporaneous exercise conditions suggests that the beneficial effects of exercise persist beyond the immediate exercise exposure period. Whether these effects of exercise also extend to adolescent-onset nicotine self-administration is examined in Chapters 2.

\section{Exercise as an intervention for nicotine addiction}

Exercise has been increasingly studied for its ability to treat psychiatric disorders including nicotine addiction. Recently, a few meta-analyses of the literature found that brief bouts of exercise in abstinent smokers acutely attenuated cigarette craving (Taylor et al. 2007; Roberts et al. 2012; Haasova et al. 2013). These effects may be due to positive effects of exercise on mood, stress, craving and withdrawal symptoms (Bock et al. 1999; Ussher et al. 2003). However, the long-term effect of exercise in maintaining abstinence in adults is less clear and requires more rigorous effort and attention in the 
field (Ussher et al. 2012). In adolescents, a two pilot studies have found that including an exercise component within a behavioral drug treatment program further decreases rates of smoking with increased success rates observed as far out as 6 months from treatment (Horn et al. 2011; Collingwood et al. 2000). These studies hint that exercise may be an effective intervention for cigarette smoking in adolescents. However, further work is necessary to determine if exercise can prevent relapse in adolescents that are addicted to nicotine and abstaining from use and to determine a causal effect of exercise without other behavioral interventions and/or sociocultural factors.

Although not yet examined for nicotine, preclinical studies indicate that exercise may be effective in reducing relapse vulnerability for other drugs of abuse. As mentioned before, relapse vulnerability is modeled in animals using extinction and reinstatement paradigms. Concurrent access to voluntary wheel running exercise during extinction and reinstatement sessions has been shown to significantly attenuate cocaine-seeking in females (Zlebnik et al. 2010). Similarly, unlimited voluntary wheel running available contemporaneously with extinction and reinstatement sessions resulted in significantly attenuated cue-induced and cocaine-primed reinstatement in both males and females (Smith et al. 2012). When exercise was made available only during abstinence from cocaine self-administration, it was found to significantly attenuate subsequent cocaineseeking during reinstatement in males (Lynch et al. 2010; Peterson et al. 2013). Importantly, in contrast to the other studies, these latter results were obtained under modest exercise conditions ( $2 \mathrm{hr} /$ day access $)$ suggesting that even a modest level of exercise can protect against relapse. Taken together, these findings support the idea that 
exercise has the ability to reduce relapse vulnerability in both males and females with effects that persist beyond the immediate exercise exposure. Chapter 3 of this dissertation will determine whether these effects of exercise extend to adolescent-onset nicotine selfadministration in males and Chapter 4 will examine potential sex differences in these effects.

Neurobiology of nicotine addiction

Addiction is a disease that progresses from initial experimentation to compulsive use and there are often are periods of abstinence followed by resumption of use. Accordingly, the neurobiology underlying addiction changes as one transitions through the phases of the addiction process. The mesolimbic dopamine pathway, commonly referred to as the "reward pathway," is the circuitry believed to mediate motivated behavior for drug rewards. Persistent administration of psychoactive drugs results in modulation of this circuitry. In a simple model, the major components of the reward pathway include the ventral tegmental area, nucleus accumbens, and cortical regions including the prefrontal cortex. Dopamine neurons of the ventral tegmental area send projections to the nucleus accumbens and the prefrontal cortex. The medial prefrontal cortex also sends glutamatergic projections to the nucleus accumbens in a region specific manner (Voorn et al. 2004). Specifically, the ventral prefrontal cortex projects primarily to the ventral medial region of the nucleus accumbens termed the shell. The shell region surrounds the central core region which receives glutamatergic projections from the 
dorsal medial prefrontal cortex. Nicotine-induced changes to these connections during the initiation, maintenance of use, abstinence and relapse are discussed in this section.

The initial exposure to drugs of abuse leads to dopamine overflow in the nucleus accumbens, which is believed to mediate their rewarding or reinforcing effects. Microdialysis studies have demonstrated that a single exposure to ethanol, nicotine, cocaine, amphetamine, and other drugs of abuse significantly increases extracellular dopamine concentrations in the nucleus accumbens (Imperato and Di Chiara 1986; Imperato et al. 1986; Di Chiara and Imperato, 1988; Carboni et al. 1989). Nicotine induces dopamine release in the nucleus accumbens via nicotinic acetylcholine receptors on VTA neurons (Corrigall et al. 1994; Nissell et al. 1994; Calabresi et al. 1989). Similar to other drugs of abuse, the initial rewarding effects of nicotine are believed to be due to dopamine release preferentially in the shell versus the core region of the nucleus accumbens. A single non-contingent exposure to cocaine, morphine amphetamine or nicotine results in robust increase in extracellular dopamine in the nucleus accumbens shell region and to a lesser extent in the core region (Pontieri et al. 1995, 1997). Repeated self-administration of nicotine during 1-hour sessions results in sensitized response to dopamine release in both the nucleus accumbens shell and core regions (Lecca et al. 2006). Thus, as self-administration continues, the dopamine response increases in both regions of the nucleus accumbens suggesting that they are both involved in nicotine selfadministration. In parallel with the neurochemical effect of drug exposure, the initial reinforcing properties of drugs of abuse including nicotine are blocked by disruption of dopamine projections to the nucleus accumbens (Lyness et al. 1979; Roberts and Koob 
1982; Corrigall et al. 1992; Caine and Koob 1994). Taken together, from initial exposure to maintenance of use, there appears to be a gradual increase in dopamine transmission in the nucleus accumbens.

Chronic drug use is often associated with escalated and uncontrolled drug intake in humans, which is best modeled using prolonged self-administration paradigms as these lead to an escalation of intake and the development of dependence over time (Ahmed and Koob 1998; O’Dell and Koob 2007). Although the neurobiological changes associated with extended access self-administration have not been examined with nicotine, studies with cocaine suggest that escalation of cocaine self-administration is associated with dopamine hypofunctionality in the mesolimbic pathway (Ahmed et al. 2003; Ahmed and Koob 2004; for review see, Melis et al. 2005). Escalation of cocaine self-administration under prolonged access conditions has been associated with higher levels of extracellular dopamine (Ahmed et al. 2003) but decreased function or number of dopamine receptors (D1- and D2-like) in the nucleus accumbens as compared to non-escalating short access conditions (Ahmed and Koob 2004). Similarly, during acute withdrawal from chronic high levels of cocaine administration, spontaneous activity of dopamine neurons is decreased in the VTA and basal levels of dopamine are lower within the nucleus accumbens (Koeltzow and White 2003). A significant role of glutamate in the mesolimbic pathway emerges after extended access to cocaine self-administration as well. Following extended but not short access to cocaine self-administration an increase in the autoregulatory presynaptic metabotropic glutamate receptor $2 / 3$ (mGluR2/3) function has been observed (Hao et al. 2010), suggesting enhanced negative modulation 
of glutamate release. Furthermore, a significant decrease in the postsynaptic metabotrobic glutamate receptor 5 (mGluR5) receptors is observed in nucleus accumbens after extended access self-administration as compared to short-access self-administration (Hao et al. 2010), suggesting a decrease in the glutamate transmission through this receptor. Basal levels of extracellular glutamate and glutamate function have also been observed in the ventral medial prefrontal cortex following extended access self-administration (BenShahar et al. 2012). Thus, unlike low levels of cocaine use observed earlier in the addiction process, an increase in cocaine use results in a hypofunctionality of dopamine as well as dysregulation of glutamatergic signaling in the mesolimbic pathway. Whether extended nicotine self-administration also results in similar neurobiological changes is not yet known.

In contrast, prolonged abstinence from nicotine is believed to result in an increase in sensitization of dopamine signaling and glutamatergic plasticity within the mesolimbic pathway, and these changes are believed to underlie lasting behavioral vulnerabilities. In particular, glutamate projections from the medial prefrontal cortex to the nucleus accumbens core region are thought to be particularly important for reinstatement drugseeking for many drugs of abuse including nicotine (Everitt and Robbins 2005; FischerSmith et al. 2012; Hotsenpiller et al. 2001; Kalivas and Volkow 2005; McFarland et al. 2003; Gipson et al. 2013). There is evidence that the ventral medial prefrontal cortex and its nucleus accumbens shell target are important for reinstatement of drug-seeking (Koya et al. 2009; Bossert et al. 2013); however, most of these data are from studies with cocaine and not nicotine. Abstinence from cocaine and amphetamine has also been found 
to induce long-lasting structural plasticity observed within 24 hours, 2 weeks, 1 month and 3.5 months in the medial prefrontal cortex and nucleus accumbens (Robinson and Kolb 1997; 1999; 2004; Ferrario et al. 2005; Heijtz et al. 2003; Kolb et al. 2003;

Crombag et al. 2005; Robinson et al. 2001; Li et al. 2004; Norrholm et al. 2003). Abstinence from experimenter administered nicotine has yielded similar results with observed increases in dendrite length and dendritic spine density in the medial prefrontal cortex and nucleus accumbens shell region (Brown and Kolb 2001; Hamilton and Kolb 2005; McDonald et al. 2005; 2007; Bergstrom et al. 2008). To date, only 1 study has examined the effect abstinence-induced structural plasticity following adult-onset nicotine self-administration, which found an increase in spine head diameter size in the nucleus accumbens core region associated with nicotine-seeking (Gipson et al. 2013). In Chapter 5 of this dissertation, abstinence-induced structural plasticity associated with nicotine-seeking in an adolescent-onset model is examined within the nucleus accumbens core and shell regions.

Finally, the molecular mechanisms underlying the effectiveness of exercise as a prevention and intervention for nicotine addiction are largely unknown. In animal models, exercise has been shown to modulate dopamine and glutamate release within the striatum as well as effect structural plasticity in the hippocampus. It is possible that the behavioral effects of exercise are mediated by exercise serving as a non-drug reward that produces similar neurobiological effects to reduce vulnerability to drug use initiation and relapse vulnerability. In Chapter 5 of this dissertation, the effect of exercise structural plasticity associated with nicotine-seeking is also examined. In Chapter 6, the hypothesis 
that the efficacy of exercise as a prevention and intervention is due to its ability to prevent neurobiological changes essential for the development of drug use initiation and relapse vulnerability is discussed further.

In summary, preclinical and clinical evidence indicates exercise may be an effective prevention and intervention for nicotine addiction. However, remaining questions involving the specificity of exercise in preventing acquisition and nicotineseeking following abstinence in an adolescent-onset model will be addressed in this dissertation. Due to disparities in the effectiveness of current tobacco cessation therapies in women, sex-differences in the effect of exercise as an intervention will also be explored. Furthermore, structural plasticity within the mesolimbic pathway as a mechanism for the efficacy of exercise in attenuating nicotine-seeking will be investigated. 


\section{CHAPTER 2: \\ Wheel running exercise as a prevention for initiation of nicotine use}

\section{Introduction}

Early smoking initiation is associated with heavy smoking (Taioli and Wynder 1991), nicotine dependence (Breslau et al. 1993; Van De Ven et al. 2010), and difficulty quitting later on in life (Breslau et al. 1996; Khuder et al. 1999) suggesting that the earlier one begins to smoke, the more likely they will die from smoking-related diseases. Although rates of smoking initiation among adolescents have decreased over the years, rates of use are still alarmingly high among adolescents. The Substance Abuse and Mental Health Services Administration (2013) estimated that in the past year, 3 million adolescents (ages 12-17) smoked cigarettes and each day 3,701 adolescents smoked for the first time. Preventing or postponing smoking initiation could greatly reduce smokingrelated deaths.

Clinical and preclinical data suggest that physical activity may protect against the initiation of drug use. Epidemiological studies have consistently demonstrated that adolescents involved in some sort of physical activity are less likely to be current smokers as compared to their less active peers (for a review see Lynch et al. 2013). A major confound in this literature is that most physically active teens participate in organized sports. Since some reports have found that participation in specific sports or type of sport may increase risk for use of smokeless tobacco, alcohol, or other drug use (Pate et al 2000; Terry-McElrath et al. 2011; Mattila et al. 2012; Castrucci et al. 2004; Kirkcaldy et al. 2002; Moore and Werch 2005; Rainey et al. 1996; Aaron et al. 1995; for 
review see Lisha and Sussman 2010), it is likely that psychosocial influences associated with being an athlete also affect drug use. Further work is necessary to determine if exercise, without social influences, can prevent drug use initiation.

Factors that may delay or prevent the initiation of regular drug use in humans can be difficult to determine in prospective studies since it is unethical to expose drug-naïve individuals, particularly adolescents, to drugs. The use of animal models circumvents this issue and, like in humans, many variables have been shown to influence the risk of drug acquisition, the transition from initial exposure to regular use (Cambell and Carroll 2000). Acquisition is usually modeled in drug and experimentally naïve animals given non-contingent infusions and then allowed to respond for drug in operant sessions. In rats, wheel running, a model of aerobic exercise, has been shown to be effective in reducing acquisition of cocaine and methamphetamine self-administration (Smith and Pitts 2011; Engelmann et al. 2013) suggesting that there is a biological basis for the effect of exercise in preventing drug use. Wheel running exercise during cocaine selfadministration has also been shown to decrease intake and drug-seeking in other phases of addiction such as maintenance of use and relapse (Cosgrove et al. 2002; Lynch et al. 2010; Zlebnik et al. 2010; Smith et al. 2012). However, no studies have examined the effect of wheel-running exercise on the acquisition of nicotine self-administration. Thus, the goal of this study was to test the hypothesis that voluntary wheel running exercise would prevent the initiation of nicotine self-administration in adolescent rats. To this end drug naïve adolescent rats were permitted daily bouts of wheel running exercise followed by nicotine self-administration sessions under acquisition testing conditions. 


\section{Methods}

Animals

Male Sprague-Dawley (Charles River Laboratories, Portage, ME, USA) rats ( $\mathrm{N}=$ 22) were shipped on day of weaning (postnatal day 21) and arrived at the laboratory on postnatal day 22. Upon arrival, animals were individually housed in self-administration chambers, and were maintained on a 12 hour light/dark cycle with ad libitum access to water and food except during exercise sessions during which only water was available. The Animal Care and Use Committee at the University of Virginia approved all procedures and were in accordance with the guidelines set by the National Institutes of Health.

Apparatus

Individual $31 \mathrm{~cm}$ x $24 \mathrm{~cm}$ x $21 \mathrm{~cm}$ self-administration chambers (ENV-008CT, Med Associates, St. Albans, VT, USA) were equipped with a house light (4.76 W), water bottle holder, food-hopper, retractable active (drug-associated) lever, a light (4.74 W) above the active lever, and a stationary inactive lever. Each chamber was centered within a ventilated sound-attenuating box (ENV-018M, Med Associates, St. Albans, VT, USA) along with a pump (PHM-100, Med Associates, St. Albans, VT, USA). A 10-ml drug syringe was mounted on the pump and connected to Tygon tubing that attached to swivel (Instech Laboratories Inc., Plymouth Meeting, PA, USA) embedded in a counterbalanced metal arm above the chamber. A polyethylene tube encased in a metal spring (C313CS; PlasticsOne, Roanoke, VA, USA) was attached to the swivel and to a 22-gauge guide 
(C313G; PlasticsOne, Roanoke, VA, USA) embedded within an infusion harness (CIH95AB; Instech Laboratories Inc., Plymouth Meeting, PA, USA) the animal wore following surgery and thereafter until study completion. The house light was illuminated from 0700 to 1900 daily to maintain a 12 hour light dark cycle. Running wheels (ENV046; Med Associates, St. Albans, VT, USA) with polycarbonate cage attachment were equipped with a revolutions counter.

Surgery

On postnatal day 28 , rats underwent surgery to implant a chronic indwelling silastic catheter (0.51 and 0.94 mm o.d.; Dow Corning Corporation) into the right jugular vein as described previously (Lynch 2008). Specifically, rats were anesthetized with a combination of dexmedetomidine $(0.2 \mathrm{mg} / \mathrm{kg})$ and ketamine $(40 \mathrm{mg} / \mathrm{kg})$. Approximately $1.6-2.0 \mathrm{~cm}$ of the catheter was inserted into the vein with the tip resting above the opening of the right atrium and was anchored in place with suture. The other end was led subcutaneously to a small incision between the scapulae and was connected to a metal cannula within a silicone harness that the animal wore for the remainder of the experiment. Rats were given ketoprofen $(2-5 \mathrm{mg} / \mathrm{kg}$, subcutaneous) and gentamicin (5.5 $\mathrm{mg} / \mathrm{kg}$, i.v.) on the day of surgery and for the 2 subsequent days. Catheter patency was assessed by flushing a small amount of heparinized saline into the catheter and pulling back to check for the presence of blood. This check was conducted prior to selfadministration sessions on the 2 days following surgery and every Monday, Wednesday, and Friday thereafter. Rats were given 2 days to recover from surgery. 
Drug

Nicotine bitartrate (Sigma-Aldrich, St. Louis, MO, USA) was dissolved in $0.9 \%$ sterile saline ( $\mathrm{pH} 7.4$ ) and passed through a microfilter; the doses are expressed as the free base weight. A single moderate dose (10 $\mu \mathrm{g} / \mathrm{kg} /$ infusion $)$ of nicotine was selected based on previous work demonstrating this dose leads to rapid and maximal rates acquisition of nicotine self-administration in adolescent males (Lynch 2009). Infusion duration was based on each individual's weight $(0.1 \mathrm{ml} / \mathrm{sec})$, which was adjusted 3 times/week. Nicotine solution was stored in the dark at $4^{\circ} \mathrm{C}$ but was available at room temperature during self-administration.

\section{Wheel-running exercise}

Rats were randomly assigned to either a sedentary $(n=12)$ or an exercise condition $(\mathrm{n}=10)$. Sedentary controls included a group that did not have access to a wheel (no wheel; $\mathrm{n}=6$ ) and as a control for environmental enrichment, a group that had access to a running wheel that was stationary (locked wheel; $n=6$ ). Figure 1 outlines the experimental timeline. Prior to surgery, on postnatal days 23-24, rats were acclimated to their assigned wheel condition for 2 hours (0930-1130). Beginning on postnatal day 30, rats were removed from their self-administration chambers 2.5 hours prior to daily selfadministration sessions (0930) and placed in a polycarbonate cage either with (locked and unlocked) or without (no wheel) a wheel attachment for 2 hours. Rats were then moved back to their self-administration chambers (1130) and they were attached for nicotine 
self-administration. During the 2 hour access, both wheel groups could move freely between the wheel and the polycarbonate cage

Acquisition of nicotine self-administration

At noon, 30 minutes after the returning to the chamber, nicotine selfadministration under a fixed ratio (FR) 1 schedule began. Daily sessions initiated with the presentation of the active lever into the chamber. Responses on the active lever were reinforced under a fixed ratio (FR) 1 schedule and each infusion was paired with the illumination of a light above the active lever. Sessions were terminated when the rat obtained all 20 infusions that were available or after 21.5 hours. Acquisition was defined as 2 consecutive days of 20 infusions with a 2:1 preference of the active lever over the inactive lever.

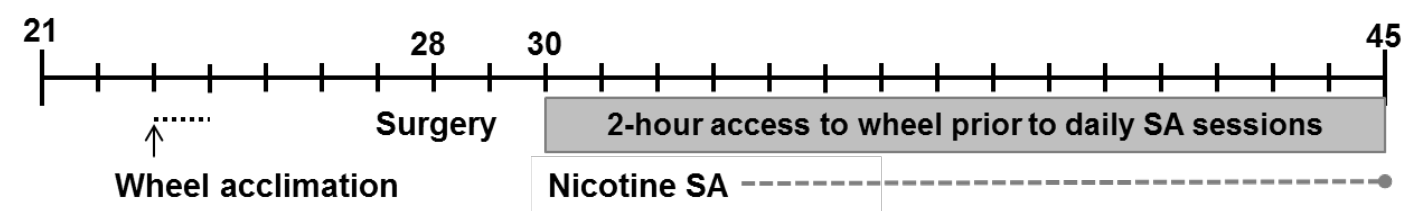

Figure 1 Experimental timeline used to examine the effect of exercise on nicotine selfadministration in adolescent rats. Rats were given 2 hour access to their wheel exercise or sedentary condition each day prior to SA beginning on postnatal day 30 . 
Data analysis

Rate of acquisition and percentage of rats acquiring were compared between wheel condition groups using the Kaplan-Meier survival analysis followed by MantelCox rank statistic. The two groups of sedentary controls (i.e., the no wheel and locked wheel groups) were combined into one sedentary group since no differences were observed between these groups in any measures examined in this study (i.e. body weights and rates of acquisition; also see Figure 2). Weights at the beginning (postnatal day 30) and at the end (postnatal day 44) were compared between groups using an ANOVA and are reported as the mean \pm SEM. Statistics were run using SPSS 20 with the alpha defined as 0.05 .

\section{Results}

No difference in rates of acquisition or the percent of the total group reaching acquisition criteria was observed between the locked and no wheel groups (Fig 2). The percent group reaching the acquisition criteria in each group was $67 \%$. Of the rats that acquired, it took on average $3.0 \pm 1.1$ days for those in the locked wheel condition and $4.3 \pm 1.3$ days for those in the no wheel condition to reach the criteria. Weights were also similar between locked and no wheel groups at the beginning $(96 \pm 3$ versus $94 \pm 5 \mathrm{~g}$, respectively) and at the end of this experiment (203 \pm 6 versus $194 \pm 9$ g, respectively). Thus these groups were combined (sedentary) for the comparison with the wheel-running exercise condition. 


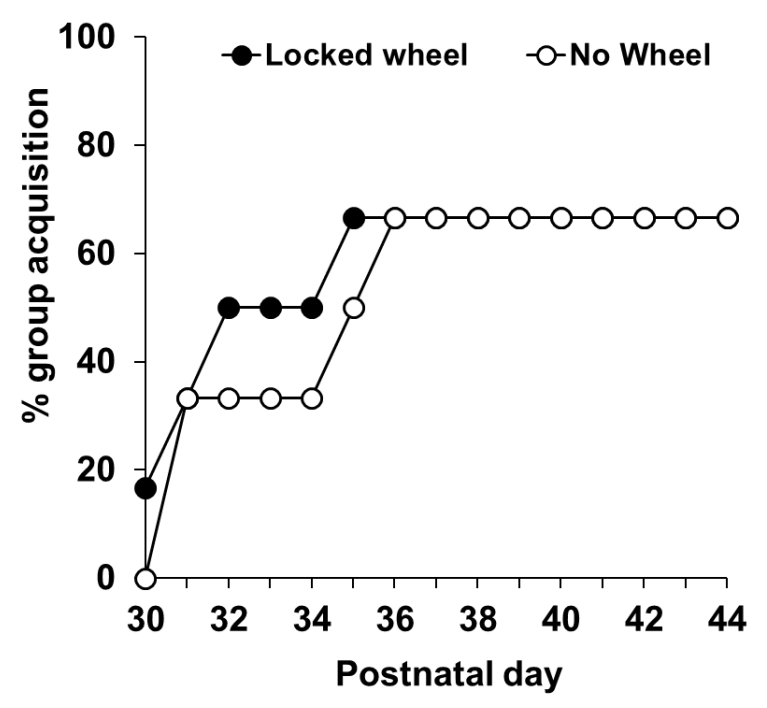

Figure 2 Acquisition of nicotine self-administration in male adolescent rats with exposure to a locked wheel (black circles) or no wheel (white circles) conditions for 2 hours prior to each self-administration session.

A greater percentage of rats in the sedentary group acquired nicotine selfadministration as compared to rats in the exercise group (Fig 3). Acquisition of nicotine self-administration was achieved by 8 of 12 (67\%) rats in the sedentary group, but only 2 of $10(20 \%)$ rats in the exercise group. A survival analysis revealed a significant effect of exercise condition $\left(\chi^{2}=4.11, \mathrm{p}<0.05\right)$ indicating a significant difference in percent group acquisition. However, the rate of acquisition criteria was similar between the sedentary and exercise groups. Specifically, in the exercise condition, the 2 rats acquired on day 2 and 3 and in the sedentary condition, rats acquired in an average of $3.6 \pm 0.8$ days. All rats gained weight over the course of the experiment with no differences observed between the exercise or sedentary groups on initial $(93 \pm 3$ versus $94 \pm 9 \mathrm{~g}$, respectively) or final (193 \pm 10 versus $199 \pm 5 \mathrm{~g}$, respectively) weights. Thus the differences observed in acquisition are not likely due to differences in weight. 


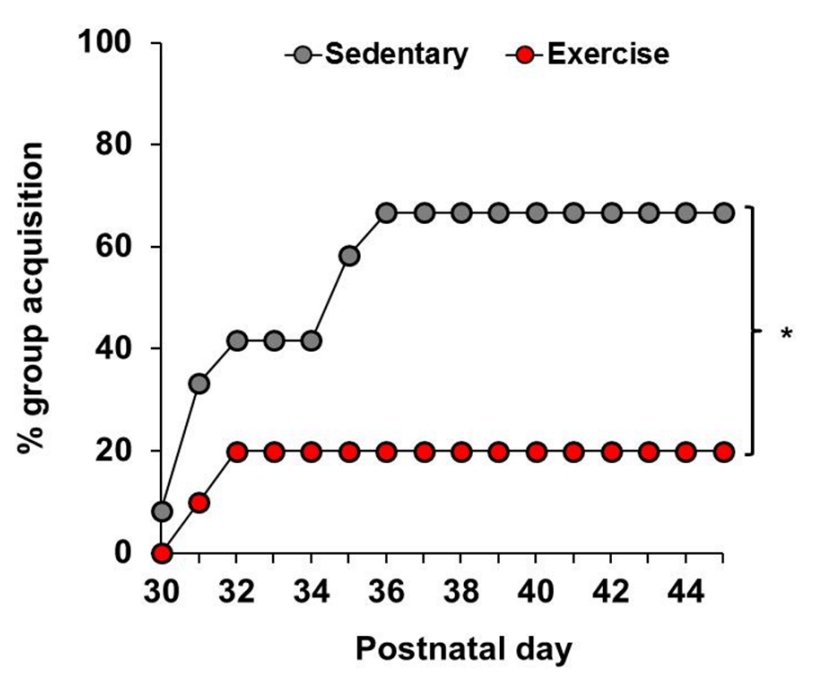

Figure 3 Percent group reaching nicotine self-administration acquisition criteria in male adolescent rats with exposure to sedentary (locked or no wheel condition; gray circles) or exercise (unlocked wheel; red circles) conditions for 2 hours prior to each selfadministration session. Asterisk indicates a significant difference between the wheel conditions. The average number of days to reach acquisition criteria for the sedentary animals is represented with the black bar.

\section{Discussion}

The goal of this study was to determine if voluntary exercise would prevent the initiation of nicotine self-administration in adolescent rats. In support of our hypothesis, significantly fewer animals in exercise group acquired nicotine self-administration as compared to those in the sedentary group. These results appear to be attributable to exercise and not environmental enrichment since the locked wheel group, which was housed in a similar environment to the exercise group, was not different from the no wheel controls. Taken together, these findings suggest that exercise in the running wheel attenuates the initiation of nicotine self-administration in adolescent rats.

This study is the first to demonstrate a preventative effect of wheel running exercise in adolescents on nicotine self-administration. Our findings are consistent with a 
previous report demonstrating that wheel-running exercise prevents acquisition of cocaine self-administration (Smith and Pitts 2011). In this previous study, rats were permitted to run 24-hours/day during a prolonged exercise training phase that spanned adolescence and into adulthood and they were able to run in their home cages following self-administration sessions. Other reports have demonstrated that exercise can decrease cocaine self-administration when the exercise is concurrently available as an alternative reinforce during self-administration sessions (Cosgrove et al. 2002; Zlebnik et al. 2012). The current report suggests that extensive training is not necessary for the beneficial effects of wheel-running exercise on acquisition of drug self-administration to emerge and that it need not be concurrently available with nicotine self-administration. Similarly, Smith and Witte (2012) demonstrated that wheel running exercise was effective in reducing the positive reinforcing effects of cocaine when initiated contemporaneously with self-administration, but that if exposure to wheel running was only available prior to self-administration it was not effective. Although, the current findings suggest that wheel running exercise decreases the positive reinforcing effect of nicotine in adolescent male rats, further is research is necessary using more demanding schedules of reinforcement (e.g. escalating FR or progressive ratio schedule), as has been demonstrated with cocaine (Smith et al 2008; Smith et al. 2011), to prove this mechanism for the effect of wheel running exercise.

In the literature examining the effects of wheel running exercise on drug selfadministration there have been inconsistencies on control conditions. Some studies utilized a locked wheel condition (Lynch et al. 2010; Zlebnik et al. 2010, 2012; Sanchez 
et al. 2013a) and others used a polycarbonate cage condition without a wheel (Smith et al. 2008, 2011, 2012; Smith and Pitts 2011, 2012; Smith and Witte 2012). The locked wheel condition controls for the presence of the wheel and the environmental enrichment this might provide; however, under these conditions, it is possible that animals may perform "alternative" forms of exercise within the wheel (e.g., climbing and hanging). While the polycarbonate cage condition without a wheel eliminates the "alternative exercise" issue it does not account for potential environmental enrichment effects. Our work is novel in that we used both control groups and found no difference between them in adolescent males. Therefore, the effect observed in the unlocked wheel group was not due to environmental enrichment provided by the wheel. This work also suggests either control (no wheel or locked wheel) is appropriate for examining the effects of wheel running exercise in adolescent males.

In human adolescents it is difficult to determine whether the suppressive effect of physical activity on smoking initiation is due to social influences or biological effects. The robust preventative effect of wheel running exercise on nicotine self-administration that we observed in adolescent male rats supports the notion that the biological effects of exercise may at least in part explain the negative association of physical activity and smoking in human adolescents. Further research is needed to determine if there are sex differences in this preventative effect of exercise and if it extends to other phases of addiction. 


\section{CHAPTER 3: \\ Wheel running during abstinence as an intervention for nicotine relapse}

\section{Introduction}

Cigarette smoking is the leading cause of preventable death in the United States and is a major health concern worldwide (Danaei et al. 2009; World Health Organization 2003). Most smokers initiate use during adolescence and those that do find it more difficult to quit later in life (Breslau and Peterson 1996). Adolescents are also known to progress to addiction more rapidly despite smoking less than adults (Tanski et al. 2004). Although rates of smoking have tapered off over recent decades, an alarming number of individuals begin smoking each year, and these rates are on the rise among adolescents (i.e., 1.5 million in 2009 compared to 1.3 million in 2002; SAMHSA 2010). Although there are FDA approved cessation treatments for nicotine addiction, none have been approved for adolescent populations, and the use of such pharmacotherapies is controversial in adolescents due to ongoing neurodevelopment (Kaplan and Ivanov 2011).

Exercise appears to be a promising non-pharmacological treatment for nicotine addiction that may be useful for the vulnerable adolescent population. Clinical studies in adults have shown that acute bouts of exercise decreases cigarette craving, withdrawal symptoms, and cue-elicited craving (Bock et al. 1999; Taylor and Katomeri 2007; Janse Van Rensburg et al. 2009, Haasova et al. 2012). In animals, wheel running, a model of aerobic exercise, appears to have both and short and long-term beneficial effects. Specifically, wheel running has been found to effectively reduce the self-administration 
of other psychostimulant drugs such as cocaine and methamphetamine and to decrease drug-seeking when contemporaneously available (Miller et al. 2012; Zlebnik et al. 2012; Smith and Pitts 2011; Smith et al. 2011; Zlebnik et al. 2010; Smith et al. 2008; Cosgrove et al. 2002). Wheel running during abstinence has also been shown to decrease subsequent cocaine-seeking even when not contemporaneously available (Lynch et al. 2010). Much less information is available on the effects of wheel running in adolescents and on nicotine self-administration.

The purpose of this study was to determine if wheel running during an abstinence period would prevent subsequent nicotine-seeking in rats that began self-administering nicotine during adolescence. An extended access paradigm was used to approximate human access conditions (Paterson and Markou 2004; O’Dell et al. 2007). Specifically, rats given extended access to nicotine (23-hr/day) have been reported to achieve daily nicotine levels that are comparable to those observed in humans (i.e. $0.18-1.38$ versus 0.14-1.14 mg/kg nicotine, in rats and humans, respectively; Valentine et al. 1997). Importantly, such conditions also lead to the development of physical dependence (i.e. withdrawal symptoms; O’Dell and Koob, 2007) and after prolonged abstinence; produce increased subsequent nicotine-seeking (e.g., increased drug-seeking behavior and increased motivation to obtain the drug; Abdolahi et al. 2010). The effects of 2-hr/day access to a running wheel on nicotine-seeking were assessed at a time when levels of nicotine-seeking are known to be high (Abdolhai et al. 2010) using a within-session extinction/cue-induced reinstatement paradigm. Two doses of nicotine were used in this study to model light versus moderate-to-high levels of consumption. Additional groups of 
rats were given access to saline infusions in order to determine whether nicotine was functioning as a reinforcer under these extended access conditions. Additionally, given previous findings showing that light/auditory cues have reinforcement value, these saline controls established baseline levels of responding in the presence and absence of cues and allowed us to then determine whether prior nicotine self-administration affects subsequent extinction and reinstatement responding. These saline groups also served as a control for nonspecific effects of wheel running on subsequent lever responding during extinction and reinstatement. We expected to find a differential effect of wheel running in attenuating nicotine-seeking, with the greatest benefit seen in rats that formerly selfadministered the lower nicotine dose since these rats were expected to self-administer less nicotine and have lower levels of subsequent nicotine-seeking as compared to rats that formerly self-administered the higher dose.

\section{Materials and Methods}

\section{Animals}

Male Sprague-Dawley (Charles River Laboratories, Portage, ME, USA) rats $(\mathrm{N}=55)$ arrived at the laboratory on postnatal day (PND) 22 with nicotine selfadministration taking place from PND 30 to PND 45. Upon arrival, animals were individually housed in self-administration chambers within sound attenuating boxes (Med Associates, St Albans, VT, USA). The self-administration apparatus design was the same as that used in Chapter 2 (pg 21). Rats were maintained on a 12-hour light/dark schedule (lights on at 0700 and off at 1900) and had free access to food and water throughout the 
experiment. All procedures were approved by the Animal Care and Use Committee at the University of Virginia and were in accordance with NIH guidelines.

Lever-press training

To ensure rapid acquisition of nicotine self-administration during the narrow window of adolescence, rats were pre-trained to lever-press for sucrose pellets $(45 \mathrm{mg})$ as described previously (Lynch 2008). Briefly, on PND 25, rats were permitted daily $23-$ hour access to lever press for sucrose pellets under a fixed ratio 1 (FR1) schedule, in which a response on the active lever resulted in the delivery of a sucrose pellet from an automated food hopper. No light or auditory cues were used in these pre-training sessions. Pre-training was terminated after 2 days of responding for 50 or more sucrose pellets. Animals were weighed upon arrival, prior to surgery, for the 2 days following surgery and 3 times a week thereafter.

Drug

Nicotine bitartrate (Sigma-Aldrich, St. Louis, MO, USA) was dissolved in $0.9 \%$ sterile saline ( $\mathrm{pH}$ 7.4) and passed through a microfilter; the doses are expressed as the free base weight. Two doses of nicotine were used in this study; a low ( $5 \mu \mathrm{g} / \mathrm{kg} /$ infusion) and a moderate (10 $\mu \mathrm{g} / \mathrm{kg} /$ infusion) dose were selected because our pilot studies indicated these doses lead to nicotine intake that were comparable to light and moderate-to-heavy smoking, respectively. Infusions of saline or nicotine $(5 \mu \mathrm{g} / \mathrm{kg} /$ infusion and 10 $\mu \mathrm{g} / \mathrm{kg} /$ infusion) were delivered at a rate of $0.1 \mathrm{ml} / \mathrm{sec}$. Infusion duration was based on 
each individual's weight which was adjusted 3 times/week. Nicotine solution was stored in the dark at $4^{\circ} \mathrm{C}$ but was available at room temperature during self-administration.

Surgery

On PND 28, rats were implanted with a chronic indwelling silastic catheter to allow intravenous self-administration of nicotine or saline as described previously in Chapter 2 (page \#). Rats were given ketoprofen (2-5 mg/kg, subcutaneous) and gentamicin $(5.5 \mathrm{mg} / \mathrm{kg}$, i.v.) on the day of surgery and for the 2 subsequent days. To ensure the patency of the catheter, it was flushed with heparinized saline every other day prior to the daily sessions.

\section{Nicotine Self-administration}

All self-administration occurred under a FR1 schedule, in which a single response on the active lever led to an infusion of nicotine. A response on the inactive lever was recorded but had no programmed consequence. A schematic of behavioral experiments is depicted in Fig 4. Rats were initially trained to self-administer nicotine (5 or 10 $\mu \mathrm{g} / \mathrm{kg} /$ infusion, $\mathrm{N}=21$ or 18 , respectively) or saline $(\mathrm{N}=16)$ under limited access conditions. Beginning on PND 30, rats were permitted a maximum of 20 infusions/day for 5 days (first shaded box in Fig 4). Once 20 infusions had been delivered within a session, the active lever was retracted from the self-administration chamber. Animals were said to have acquired nicotine self-administration after 2 consecutive days of receiving all 20 infusions with a 2:1 preference for the active lever over the inactive 
lever. Beginning on PND 35, self-administration access was extended to 23-hours/day with an unlimited number of infusions available per session. Rats were then switched back to limited access conditions with a maximum of 20 infusions per session for 2 days prior to abstinence (second shaded box in Fig 4) in order to normalize nicotine intake within each dose condition prior to abstinence.

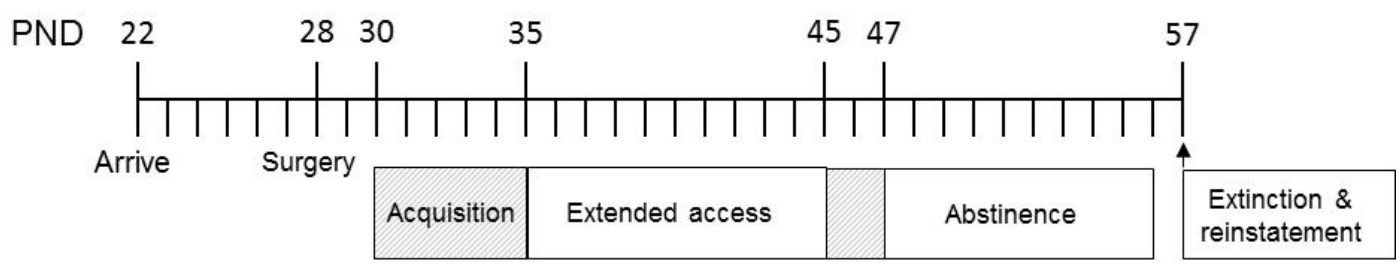

Figure 4 Schematic of behavioral protocol by postnatal day (PND). Self-administration began with a 5-day acquisition period in which rats were limited to 20 infusions/day under a fixed ratio 1 (FR1) schedule. Subsequently, rats were permitted an unlimited number of infusions for 23-hours/day during the 10-day extended access period. Next, infusions were again limited to 20 infusions/day for 2 days prior to forced abstinence. During the 10-day abstinence period rats had 2-hour access to either a locked or unlocked running wheel each day. After the last exercise session rats were returned to selfadministration boxes and the following day began a within-session extinction/cueinduced reinstatement paradigm.

\section{Wheel running}

On PND 47, rats were moved to polycarbonate cages with a wheel attachment (diameter: $35.6 \mathrm{~cm}$; Med Associates, St Albans, Vermont, USA) for the 10-day abstinence period. A prolonged duration of abstinence was selected based on a previous report demonstrating that nicotine-seeking increases, or incubates, over an abstinence 
period with high levels achieved after 7-days (Abdolahi et al. 2010). Rats were randomly assigned to either 2-hr/day access to a locked (saline $\mathrm{n}=8 ; 5 \mu \mathrm{g} / \mathrm{kg}$ dose $\mathrm{n}=11 ; 10 \mu \mathrm{g} / \mathrm{kg}$ dose $\mathrm{n}=9$ ) or unlocked (saline $\mathrm{n}=8 ; 5 \mu \mathrm{g} / \mathrm{kg}$ dose $\mathrm{n}=10 ; 10 \mu \mathrm{g} / \mathrm{kg}$ dose $\mathrm{n}=9$ ) running wheel condition. In the unlocked wheel condition rats were free to run in the wheel during the daily access sessions. Rats in the locked wheel condition were able to enter the wheel, however the wheel was stationary and they were not able to run. Wheel access sessions occurred between 1000 and 1200, during the light phase of the light/dark cycle, during which rats were free to move between wheel and their polycarbonate cage. A 2hour exercise session was selected based on previous work with cocaine demonstrating that this level access during abstinence was sufficient to reduce subsequent cocaineseeking (Lynch et al. 2010). Revolutions completed during each unlocked wheel session were recorded daily.

\section{Extinction and cue-induced reinstatement}

Following the last wheel session, animals were moved back to their operant conditioning chambers for the remainder of the day. Nicotine-seeking was then tested the following day (PND 57) under a within-session extinction and cue-induced reinstatement paradigm. Extinction responding was examined in at least 5 sessions that were each 1hour in duration until responding extinguished (defined as fewer than 15 responses in the last session). Each extinction session began with the extension of the active lever into the self-administration chamber. Responses on the active lever were recorded but had no programmed consequence. At the end of each session there was a 5-minute timeout 
period in which the active lever was retracted to separate each extinction sessions. The 1hour reinstatement session began after the final extinction session and a 5-minute timeout with the introduction of the active lever into the chamber and presentation of the discrete cues formerly associated with nicotine (i.e. the stimulus light and the sound of the pump) for 5 seconds. Each response on the active lever during this session resulted in the presentation of these cues but did not produce an infusion of nicotine or saline.

\section{Data analysis}

All data are presented as mean \pm standard error of mean (SEM). Repeated measures analysis of variance (ANOVA) was used to analyze group differences in extended access self-administration, wheel-running during the abstinence period, and lever responses during extinction and reinstatement sessions. The main dependent measures included daily number of infusions during extended access, average daily intake during extended access, daily distance run during abstinence, and total responding during the extinction and reinstatement sessions. In addition to these analyses we also further examined rates of extinction by comparing the number of sessions required to meet the extinction criterion using univariate analysis of variance. Specifically, although a minimum of 5 extinction sessions were run some animals met the extinction criterion in fewer than 5 sessions and some required 1(two rats within the $5 \mu \mathrm{g} / \mathrm{kg}$ dose locked wheel condition, two within the $5 \mu \mathrm{g} / \mathrm{kg}$ dose unlocked wheel condition, and one rat within the $10 \mu \mathrm{g} / \mathrm{kg}$ unlocked wheel condition) or more additional sessions (one rat within the 10 $\mu \mathrm{g} / \mathrm{kg}$ dose locked wheel condition). All post-hoc analyses were done using the 
Bonferroni corrected t-test. One tailed t-tests were used to test all a priori predicted comparisons. Specifically, we predicted that nicotine as compared to saline would lead to higher levels of responding under both extended access conditions and extinction/reinstatement testing conditions, that intake during extended access conditions and levels of responding during extinction/reinstatement would be highest in the 10 $\mu \mathrm{g} / \mathrm{kg}$ dose group, and that wheel running would decrease levels of extinction/reinstatement responding, particularly in the $5 \mu \mathrm{g} / \mathrm{kg}$ dose group. The Pearson correlation coefficient was used to determine the associations between wheel running and subsequent extinction and reinstatement responding. Statistical analyses were performed using SPSS (version 20) and alpha was set to 0.05 for all tests.

\section{Results}

\section{Extended access}

During the extended access period, rats that self-administered nicotine at the 5 and $10 \mu \mathrm{g} / \mathrm{kg}$ doses, obtained significantly more infusions than rats that self-administered saline (Fig. 5A; overall effect of group, $F_{2,52}=18.2, p<0.001 ; 5 \mu \mathrm{g} / \mathrm{kg}$ versus saline, $\mathrm{t}_{35}$ $=5.6, \mathrm{p}<0.001 ; 10 \mu \mathrm{g} / \mathrm{kg}$ versus saline, $\left.\mathrm{t}_{32}=6.6, \mathrm{p}<0.001\right)$. Although the number of infusions obtained did not differ between the 5 and $10 \mu \mathrm{g} / \mathrm{kg}$ groups, average nicotine intake was significantly greater for the $10 \mu \mathrm{g} / \mathrm{kg}$ group as compared to the $5 \mu \mathrm{g} / \mathrm{kg}$ group (effect of dose; $F_{1,35}=14.0, p<0.01 ;$ Fig. 5B). Importantly, within each dose, intake did not differ for rats that would later be in the unlocked and locked groups. Thus, any 
potential differences found in subsequent nicotine-seeking behavior cannot be attributed to differing levels of nicotine intake.

Inactive lever responding was comparable to the low levels of active lever responding observed among saline controls and did not differ significantly between groups. The average daily inactive lever responses during the extended access period were $22.5 \pm 6.6,31.6 \pm 6.9$, and $27.6 \pm 5.4$ for saline, $5 \mu \mathrm{g} / \mathrm{kg}$ dose, and $10 \mu \mathrm{g} / \mathrm{kg}$ dose groups, respectively. Animal weights were the same across all conditions at the onset of self-administration (saline, $102 \pm 3 \mathrm{~g} ; 5 \mu \mathrm{g} / \mathrm{kg}$ dose, $97 \pm 2 \mathrm{~g} ; 10 \mu \mathrm{g} / \mathrm{kg}$ dose, $98 \pm 3 \mathrm{~g}$ ) At the end of the self-administration period, however, the nicotine groups weighed slightly less than the saline group, although this effect did not reach statistical significance (saline, $225 \pm 6 \mathrm{~g} ; 5 \mu \mathrm{g} / \mathrm{kg}$ dose, $204 \pm 7 \mathrm{~g} ; 10 \mu \mathrm{g} / \mathrm{kg}$ dose, $213 \pm 5 \mathrm{~g}$ ).

\section{Wheel running during abstinence}

Although time-dependent changes in daily levels of running were observed where levels of running increased over time (Fig. 6A), overall levels of running did not differ between doses. A repeated measures ANOVA revealed a significant effect of day $\left(\mathrm{F}_{9,216}\right.$ $=4.3, \mathrm{p}<0.001)$, but a non-significant effect of dose and a non-significant interaction of group by day. The similarity between the doses for levels of running is further illustrated in Fig. 6B where data are plotted as average daily distance run for the 0,5 , and $10 \mu \mathrm{g} / \mathrm{kg}$ groups. 

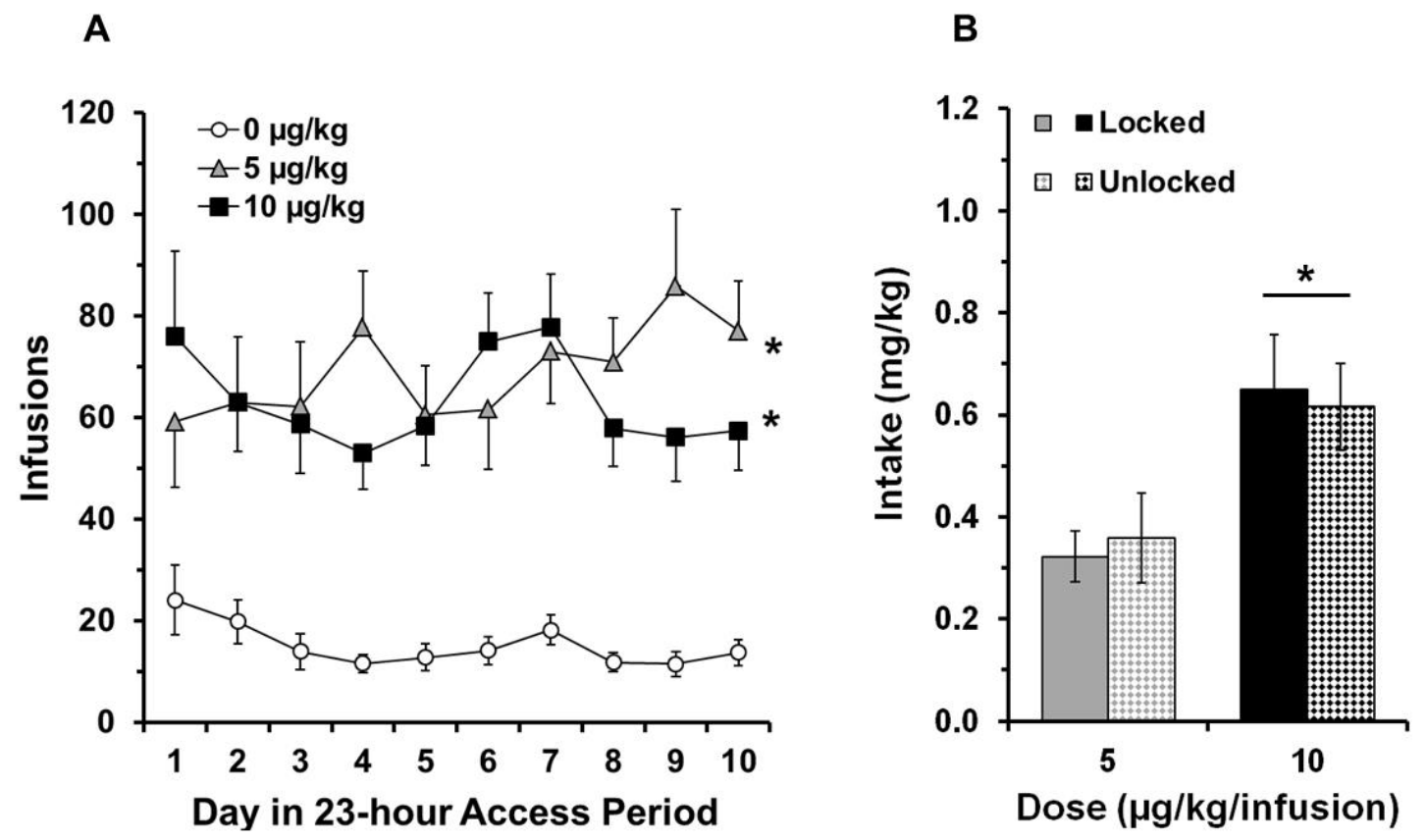

Figure 5 Nicotine is reinforcing in adolescent rats. (a) Number of infusions (mean \pm SEM) is plotted for each of the 10 days of extended access period. The open circles represent saline data points $(n=16)$, gray triangles represent the $5 \mu \mathrm{g} / \mathrm{kg}$ data points $(\mathrm{n}=$ $21)$, and black squares represent the $10 \mu \mathrm{g} / \mathrm{kg}$ data points $(\mathrm{n}=18)$. An * indicates a significant difference from saline $(\mathrm{p}<0.05)$. (b) Average daily intake during extended access (mean $\pm \mathrm{SEM}$ ) is plotted for all nicotine groups. Solid gray bar represents the locked wheel condition in the $5 \mu \mathrm{g} / \mathrm{kg}$ dose $(\mathrm{n}=11)$, gray checkered bar represents the unlocked wheel condition in the $5 \mu \mathrm{g} / \mathrm{kg}$ dose $(\mathrm{n}=10)$, black solid bar represents the locked wheel condition in the $10 \mu \mathrm{g} / \mathrm{kg}$ dose $(\mathrm{n}=9)$ and black checkered bar represents the unlocked wheel condition in the $10 \mu \mathrm{g} / \mathrm{kg}$ dose $(\mathrm{n}=9)$. The bar and * indicates a significant effect of dose $(\mathrm{p}<0.05)$ 
A

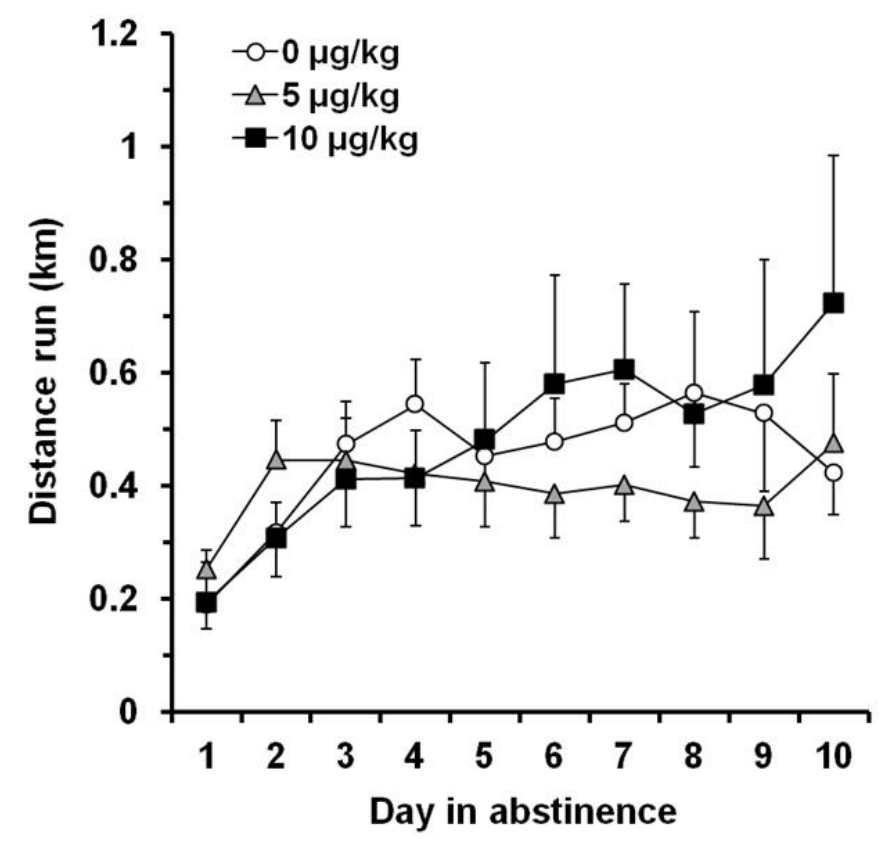

B

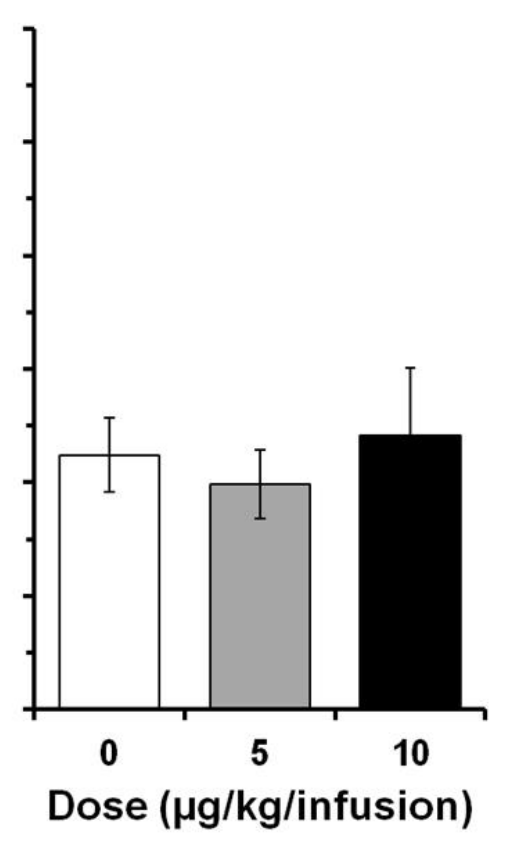

Figure 6 Nicotine self-administration does not affect wheel-running. (a) Number of revolutions (mean $\pm \mathrm{SEM}$ ) is plotted for each of the 10 days during the abstinence period. The open circles represent saline data points $(n=8)$, gray triangles represent the $5 \mu \mathrm{g} / \mathrm{kg}$ data points $(\mathrm{n}=10)$, and black squares represent the $10 \mu \mathrm{g} / \mathrm{kg}$ data points $(\mathrm{n}=9)$. (b) Average daily distance run in kilometers (mean \pm SEM) is plotted between groups. The white bar represents the saline condition, the gray bar represents the $5 \mu \mathrm{g} / \mathrm{kg}$ dose, and the black bar represents the $10 \mu \mathrm{g} / \mathrm{kg}$ dose. 


\section{Extinction and cue-induced reinstatement}

Levels of extinction responding were highest in rats that self-administered nicotine and were given access to a locked wheel during abstinence (Fig 7). A repeated measures ANOVA on responding across the 5 extinction sessions revealed a significant effect of dose $\left(F_{2,49}=6.5, p<0.01\right)$ and wheel-condition $\left(F_{1,49}=6.2, p<0.05\right)($ Fig 7$)$. Subsequent analysis of total extinction responding within each dose, revealed a significant effect of wheel condition within both the 5 and $10 \mu \mathrm{g} / \mathrm{kg}$ nicotine groups $\left(\mathrm{t}_{19}=\right.$ $2.0, \mathrm{p}<0.05 ; \mathrm{t}_{16}=1.9, \mathrm{p}<0.05$, respectively), but not within the saline group. Within the locked wheel condition, levels of extinction responding were significantly higher within both nicotine groups as compared to saline controls (effect of dose, $F_{2,25}=4.0, p<0.05$; $5 \mu \mathrm{g} / \mathrm{kg}$ versus saline, $\mathrm{t}_{17}=3.1, \mathrm{p}<0.01 ; 10 \mu \mathrm{g} / \mathrm{kg}$ versus saline, $\mathrm{t}_{15}=3.0, \mathrm{p}<0.01 ;$ Fig. 8). Similarly, under unlocked wheel conditions, both nicotine groups had higher levels of responding during extinction as compared to saline (effect of dose, $F_{2,24}=3.5, p<0.05 ; 5$ $\mu \mathrm{g} / \mathrm{kg}$ versus saline $\mathrm{t}_{16}=2.7, \mathrm{p}<0.05 ; 10 \mu \mathrm{g} / \mathrm{kg}$ versus saline $\mathrm{t}_{15}=2.4, \mathrm{p}<0.05$ ) however, no difference was observed between the two nicotine dose conditions. Extinction responding was higher in the initial sessions particularly the first session as compared to later sessions with results from the overall ANOVA revealing a significant effect of time $\left(\mathrm{F}_{4,196}=5.4, \mathrm{p}<0.001\right)$. Although no significant interaction of time by dose was observed when levels of responding were examined across the 5 sessions of extinction, analysis of the number of sessions required to meet the extinction criteria revealed a significant effect of dose $\left(F_{2,49}=4.2, p<0.05\right)$, where both nicotine groups took significantly longer to extinguish responding as compared to saline $(5 \mu \mathrm{g} / \mathrm{kg}$ versus 
saline, $\mathrm{t}_{35}=2.4, \mathrm{p}<0.05 ; 10 \mu \mathrm{g} / \mathrm{kg}$ versus saline, $\left.\mathrm{t}_{32}=3.0, \mathrm{p}<0.01\right)$. However, no overall effect of wheel condition was observed on rates of extinction. There was also no association between the levels of wheel running and subsequent extinction responding. Analysis of the inactive lever responses during extinction revealed no effect of dose or wheel condition. Inactive lever responding for locked and unlocked wheel conditions were $8.3 \pm 3.2$ and $11.0 \pm 3.4,15.9 \pm 2.8$ and $14.8 \pm 4.0,21.0 \pm 6.8$ and $6.2 \pm 2.5$ for saline, $5 \mu \mathrm{g} / \mathrm{kg}$ dose, and $10 \mu \mathrm{g} / \mathrm{kg}$ dose groups, respectively. Thus, nicotine-seeking as assessed under extinction conditions was enhanced following abstinence in both nicotine groups, and wheel running during abstinence attenuated this effect.

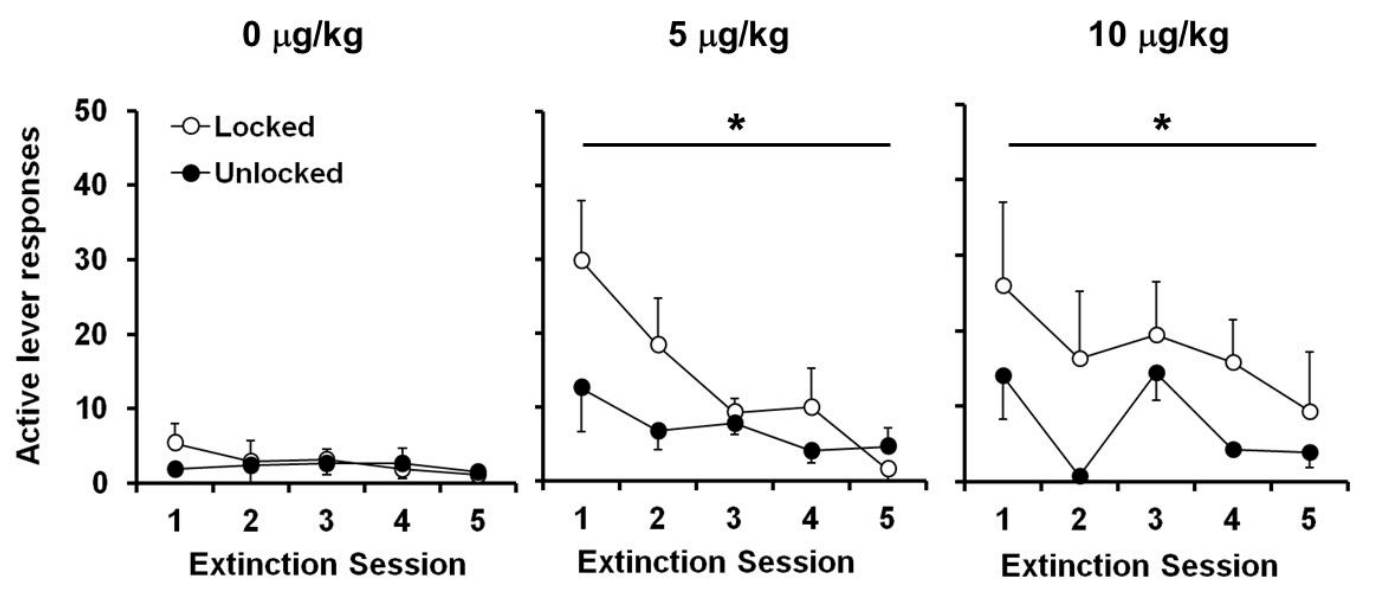

Figure 7 Wheel running during abstinence period significantly attenuates nicotineseeking during extinction. Number of responses (mean $\pm \mathrm{SEM}$ ) is plotted for each of the first 5 extinction sessions. Open circles represent the locked wheel condition (saline, $\mathrm{n}=$ $8 ; 5 \mu \mathrm{g} / \mathrm{kg}$ dose, $\mathrm{n}=11 ; 10 \mu \mathrm{g} / \mathrm{kg}$ dose $\mathrm{n}=9$ ) condition and black circles represent the unlocked wheel condition (saline, $\mathrm{n}=8 ; 5 \mu \mathrm{g} / \mathrm{kg}$ dose, $\mathrm{n}=10 ; 10 \mu \mathrm{g} / \mathrm{kg}$ dose, $\mathrm{n}=9$ ). A bar and * indicates a significant effect of exercise condition $(p<0.05)$. 


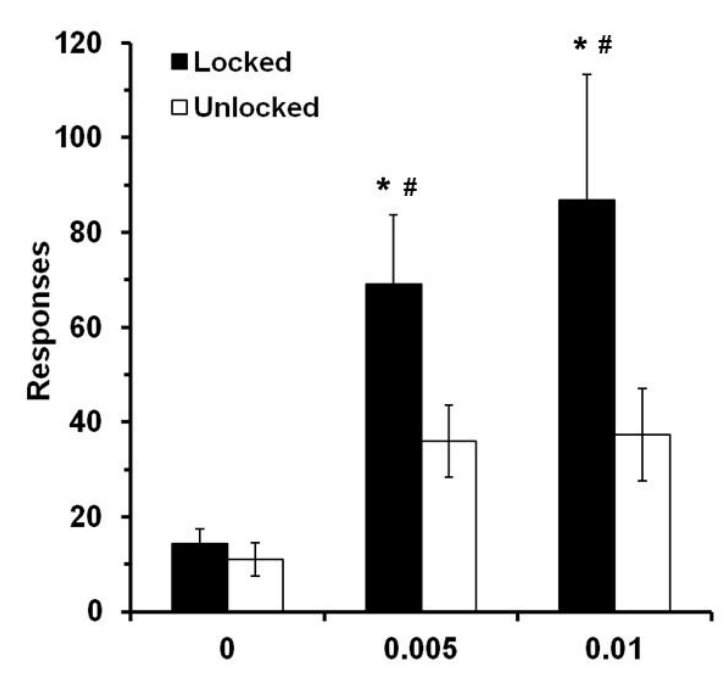

Figure 8 Wheel running effectively reduces during extinction responding in both nicotine doses. Total number of responses during the first 5 extinction sessions (mean $\pm \mathrm{SEM}$ ) is plotted by dose and wheel condition. White bars represent the locked wheel condition and black bars represent the unlocked wheel condition. The * indicates a significant difference from saline counterpart $(\mathrm{p}<0.05)$ and the \# indicates a significant difference from unlocked wheel group within dose $(\mathrm{p}<0.05)$.

In contrast to the effects seen in extinction responding, levels of reinstatement responding did not differ by wheel condition. In fact, levels of reinstatement responding were relatively low and although a significant effect of time was observed when comparing levels of responding during the last extinction session to those observed during the reinstatement session $\left(F_{1,49}=13.8, p<0.01\right.$; Table 1$)$, the interactions of time by wheel condition and time by wheel condition by dose were not significantly different. We did, however, observe a trend for a significant time by dose interaction $(p=0.053)$ with subsequent comparisons within each dose revealing significant effect of time in both the 5 and the $10 \mu \mathrm{g} / \mathrm{kg}$ doses $\left(\mathrm{F}_{1,19}=19.7, \mathrm{p}<0.001 ; \mathrm{F}_{1,16}=4.7, \mathrm{p}<0.05\right.$, respectively $)$ but not the saline, suggesting a tendency for nicotine to induce reinstatement responding. 
In order to further explore this possibility we examined responding during just the reinstatement session and found a significant effect of dose $\left(F_{2,49}=3.4, p<0.05\right)$ with reinstatement responding higher in both nicotine doses as compared to saline $(5 \mu \mathrm{g} / \mathrm{kg}$ dose versus saline $t_{35}=3.7, \mathrm{p}<0.01 ; 10 \mu \mathrm{g} / \mathrm{kg}$ dose versus saline $\mathrm{t}_{32}=2.2, \mathrm{p}<0.05$ ). Importantly, total number of responses during the last extinction session did not differ significantly by dose or wheel conditions. There was no association between the levels of wheel running and subsequent reinstatement responding. Analysis of the inactive lever responses during reinstatement revealed no effect of dose or wheel condition. Inactive lever responding for locked and unlocked wheel conditions were $0 \pm 0$ and $2.3 \pm 1.1,1.5 \pm$ 0.7 and $2.5 \pm 1.5,0.7 \pm 0.3$ and $1.0 \pm 0.8$ for saline, $5 \mu \mathrm{g} / \mathrm{kg}$ dose, and $10 \mu \mathrm{g} / \mathrm{kg}$ dose groups, respectively. Although nicotine induced subsequent reinstatement responding, levels were modest and variable and did not differ by wheel condition.

Table 1. Wheel running does not attenuate cue-induced reinstatement

\section{Last Extinction Reinstatement Responses Responses}

\begin{tabular}{|c|c|c|c|}
\hline Group & & & \\
\hline \multicolumn{4}{|l|}{$0 \mu \mathrm{g} / \mathrm{kg}$} \\
\hline Locked & $1.1(1.1)$ & $0.5(0.4)$ & \\
\hline Unlocked & $1.5(0.9)$ & $1.0(0.6)$ & \\
\hline $5 \mu \mathrm{g} / \mathrm{kg}$ & & $\#$ & \\
\hline Locked & $0.7(0.5)$ & $7.1(2.5)$ & * \\
\hline Unlocked & $1.5(0.7)$ & $9.6(2.8)$ & \\
\hline $10 \mu \mathrm{g} / \mathrm{kg}$ & & $\#$ & \\
\hline Locked & $1.4(0.8)$ & $13.6(7.7)$ & * \\
\hline Unlocked & $3.9(1.8)$ & $7.8(4.2)$ & \\
\hline
\end{tabular}

The mean $( \pm$ SEM) number of active lever responses

$*$ indicates a significant increase in responding from last extinction $(\mathrm{p}<0.05)$

$\#$ indicates a significant increase from saline $(\mathrm{p}<0.05)$ 


\section{Discussion}

The goal of this study was to determine if wheel-running during an abstinence period would attenuate subsequent nicotine-seeking in an adolescent-onset model of nicotine addiction. Consistent with our hypothesis, wheel running during abstinence significantly attenuated nicotine-seeking. However, in contrast to our hypothesis, levels of nicotine-seeking were comparable between the two dose conditions, and wheel running produced a similar decrease under both dose conditions. The effects of wheel running were also apparent under extinction conditions, but not under cue-induced reinstatement conditions where levels of responding were relatively low and variable. The present study is the first to show that wheel running effectively reduces drug-seeking in an adolescent-onset model, as well as the first to demonstrate its efficacy for nicotine.

Wheel running during abstinence attenuated subsequent extinction responding in animals that had previously self-administered nicotine. This finding is consistent with previous work in which wheel running during an abstinence period significantly attenuated subsequent cocaine-seeking in adult rats (Lynch et al. 2010). Furthermore, as was found in Lynch et al. (2010), in this study there was no association between levels of wheel running and drug-seeking. It is also important to note that the rats were permitted to run during the light phase of their light/dark cycle where the levels of wheel running are expected to be lower as compared to the dark phase. Despite the time of day and modest levels of running in the wheel, we observed a significant attenuation of extinction responding 10 days after the last self-administration session and 1 day after the last wheel running session. Taken together, these data suggest that even modest levels of exercise 
may produce a beneficial effect on nicotine-seeking that persists beyond the acute bout of exercise.

Surprisingly, levels of cue-induced reinstatement were not affected by prior wheel running. These findings are in contrast to studies with cocaine, where wheel and treadmill running have been found to decrease subsequent cocaine-seeking under both extinction and reinstatement conditions (Lynch et al. 2010; Smith et al. 2012; Thanos et al. 2013). One possible explanation for these discrepant results is that exercise differentially affects drug-seeking during extinction versus reinstatement in rodents that previously selfadministered cocaine versus nicotine. However, a more likely explanation is that the effects of wheel running during reinstatement were obscured in the present study by a floor effect. Specifically, levels of reinstatement responding under control locked wheel conditions were relatively low (mean, $9 \pm 4$ ) and variable (0-74 responses). Indeed, within the locked wheel condition, levels of reinstatement responding were roughly one third of the average observed in these same animals during the first hour of extinction, and comparable to the wheel running-attenuated levels observed during hour one of extinction within the unlocked wheel condition. The levels of reinstatement responding observed here are also much lower than those observed previously for cue-induced reinstatement following cocaine self-administration. In addition to differences in the levels of reinstatement responding induced following cocaine versus nicotine selfadministration, age may account for this floor effect. Specifically, recent findings with cocaine, show that while a cocaine prime or stressor led to robust reinstatement in adolescents, the presentation of cues did not reinstate cocaine-seeking in this age group. 
However, in the adult comparison group, cues, stress, and cocaine prime all reinstated cocaine-seeking (Anker and Carroll 2010). These findings suggest that cue-induced reinstatement is not sufficiently robust in adolescents to reveal an effect of exercise. Cueinduced reinstatement is known to rely on the prefrontal cortex (Koya et al. 2008), and given that this brain region continues to develop throughout adolescence (Counotte et al. 2011; Geidd 2004), it is possible that adolescents are less vulnerable than adults to drugseeking in response to cues. Further research is needed to address this possibility.

During the self-administration period, nicotine maintained significantly higher levels of responding than did saline, indicating that nicotine was functioning as a reinforcer in these adolescent rats. As has been previously reported with extended access conditions (O’Dell et al. 2007; Valentine et al. 1997), the higher (10 $\mu \mathrm{g} / \mathrm{kg})$ dose of nicotine maintained significantly higher levels of intake than did the lower $(5 \mu \mathrm{g} / \mathrm{kg})$ dose of nicotine. However, despite the differences in levels of intake and in contrast to our hypothesis, we found that levels of nicotine-seeking were similar between rats that had self-administered either the higher or lower dose of nicotine, and that wheel running during abstinence was equally effective at reducing nicotine-seeking during extinction between both nicotine groups. These results suggest that exercise may be equally effective in both light and moderate to heavy smokers. It should be noted, however, that because rats metabolize nicotine faster than humans, both doses of nicotine may have resulted in lower levels of nicotine in the brain as compared to those achieved in human smokers (Matta et al. 2007). Although exercise can also affect drug metabolism (Døssing. 
1985), this would not have been a contributing factor in this study because wheel running did not overlap with nicotine self-administration.

There are currently no FDA-approved drug therapies for smoking cessation in adolescent smokers. The findings from this study showing that wheel running during abstinence attenuates subsequent nicotine-seeking in an adolescent-onset model suggests that exercise may effectively prevent smoking relapse in teens. Our current findings in rats are also consistent with recent findings in adolescent humans showing that high school students enrolled in an afterschool program with an exercise component were more likely than other high school students to abstain from smoking (Horn et al. 2011). One factor to consider is that while wheel running is highly reinforcing in rats, humans have different affinities for various types of exercise (Ekkekakis et al. 2008) and may benefit most from exercise that they "like" to do. In the present study, rats that ran in the wheel showed a significant benefit as compared to rats that could only sit or climb on the wheel and thus appear to benefit from aerobic-like exercise. Future studies should explore the benefits of aerobic and non-aerobic exercise on adolescent smoking behavior. 


\section{CHAPTER 4: \\ Sex differences in wheel running as an intervention for nicotine relapse}

\section{Introduction}

Disease brought on by cigarette smoking is a major health concern worldwide accounting for approximately 5 million deaths each year (World Health Organization, 2009). Although the rate of cigarette smoking has decreased over the last decade, rates have remained high in younger populations. In the United States, approximately $10 \%$ of youth (age 12-17) and 34\% of young adults (age 18-25) are current smokers (Substance Abuse and Mental Health Services, 2011). This is concerning because people who begin smoking early in life have greater difficulty with quit attempts and are more likely to be heavy smokers in adulthood (Breslau and Peterson 1996). Despite the fact that more men than women are current smokers, smoking rates among adolescent boys and girls do not differ (SAMHSA, 2011). Female smokers are at a greater risk for smoking-related diseases and have more difficulty quitting smoking as compared to male smokers (Perkins et al. 1999). Physiological predispositions to nicotine dependence and inability to quit may be further impacted by psychosocial factors to control appetite in teens (Klesges et al. 1997; Boles \& Johnson 2001; Cavallo et al. 2010). Preclinical studies also suggest an enhanced vulnerability to nicotine addiction in females and adolescent animals (Donny et al. 2000; Lynch 2009; Torres et al. 2008; Torres et al. 2009), suggesting that their use may be suitable for addressing the biological basis of these vulnerabilities without the influence of social factors, as well as for determining potential sex-specific interventions. 
Since nicotine is the primary addictive component in tobacco products, most currently available therapies either replace nicotine or partially mimic its actions on nicotinic acetylcholine receptors. The use of such pharmacological interventions in adolescents is controversial due to ongoing neurodevelopment and there are currently no FDA approved treatments for people under the age of 18 (Kaplan and Ivanov 2011). Thus, non-pharmacological therapies, such as exercise, would be ideal for treatment of nicotine addiction in the vulnerable adolescent population. Previous work in both humans and animal models suggests that exercise may be an effective cessation aid. For example, in adult smokers, exercise during abstinence has been found to acutely reduce craving, desire to smoke, and withdrawal symptoms (Taylor et al. 2007; for review see Roberts et al. 2012; Haasova et al. 2013). Similarly, in animal models, wheel running has been found to slow the initiation of cocaine use, decrease intake, and attenuate cocaine-seeking (Cosgrove et al. 2002; Smith et al. 2008; Lynch et al. 2010; Zlebnik et al. 2010; Miller et al. 2011; Smith et al. 2011; Smith and Pitts 2011; Zlebnik et al. 2012). We recently demonstrated that voluntary wheel running during an abstinence period effectively reduced subsequent nicotine-seeking in males that began nicotine self-administration during adolescence (Sanchez et al. 2013a). Further work is necessary to determine potential sex differences in the effect of exercise during abstinence in preventing subsequent drug relapse, particularly for nicotine addiction.

The purpose of this study was to determine if wheel running during abstinence would attenuate subsequent drug-seeking in an adolescent-onset model of nicotine addiction in male and female rats and determine if its efficacy varies between the sexes. 
An extended access (23-hour) self-administration paradigm was used to model human nicotine consumption and addiction. This self-administration paradigm has previously been found to result in nicotine intake that is comparable to the average daily intake observed in humans and lead to nicotine dependence (Valentine et al. 1997; O’Dell and Koob 2007). In addition, this paradigm has been found to induce robust nicotine-seeking in adolescent and adult male rats following a prolonged (i.e. 7 or 10 days), but not short (i.e. 1 hour), abstinence period (Abdolahi et al. 2010; Sanchez et al. 2013a). To assess the potential of exercise to prevent nicotine-seeking, rats were permitted to exercise in a running wheel during a prolonged abstinence period and their behavior was subsequently examined under a within-session extinction/reinstatement paradigm to test the effects of exercise on relapse vulnerability. Adolescent females have been shown to acquire nicotine self-administration faster and to be more motivated to self-administer nicotine than adolescent males under low nicotine dose conditions (i.e. $5 \mu \mathrm{g} / \mathrm{kg}$; Lynch 2009). Thus, we hypothesized that females would self-administer more nicotine subsequently leading to greater levels of nicotine-seeking as compared to males. Based on previous data collected from our lab on the effect of wheel running in adolescent males (Sanchez et al. 2013a) and others demonstrating heightened female sensitivity to the effects of wheel running as an alternative nondrug reward (Cosgrove et al. 2002), we hypothesized that exercise would attenuate subsequent nicotine-seeking in both sexes with a greater treatment effect in females. 


\section{Materials and Methods}

\section{Subjects}

Male $(\mathrm{N}=49)$ and female $(\mathrm{N}=43)$ Sprague-Dawley rats (Charles River Laboratories, Portage, ME, USA) were weaned and shipped on postnatal day 21 and arrived at the laboratory on postnatal day 22. Upon arrival, rats were housed individually in self-administration chambers with ad libitum access to food and water and were maintained on a 12-hour light/dark cycle. The self-administration apparatus design was the same as those used in Chapter 2 (page 21). Results from a subset of the male rats $(\mathrm{N}=$ 29) were published as part of another study (Sanchez et al. 2013a) and presented in Chapter 3 with an additional 20 males added for the present study. No differences were observed between the two cohorts of male rats in any of the measures examined (rates of acquisition and levels of nicotine self-administration, wheel running, and nicotineseeking). Rats were weighed every other day and general health was monitored daily. Prior to surgery, all rats were trained on the active lever under an FR1 schedule for sucrose pellets (45 mg) as described previously in Chapter 3. All procedures were approved by The Animal Care and Use Committee at the University of Virginia and were in accordance with NIH guidelines.

Drug

Nicotine bitartrate (Sigma-Aldrich, St. Louis, MO, USA) was dissolved in 0.9\% sterile saline ( $\mathrm{pH} 7.4)$ and delivered at a concentration of $5 \mu \mathrm{g} / \mathrm{kg} /$ infusion at a rate of 0.1 $\mathrm{ml} / \mathrm{sec}$. The dose is expressed as the free base weight. This dose of nicotine was selected 
based on previous work demonstrating robust nicotine-seeking following extended access self-administration in males (Sanchez et al. 2013a; Chapter 3) and sex differences in acquisition and motivation for nicotine self-administration in adolescents at this dose (Lynch 2009). Infusion duration was based on each individual's weight, which was adjusted 3 times/week. Nicotine solution was stored in the dark at $4{ }^{\circ} \mathrm{C}$ but was available at room temperature during self-administration.

Surgery

Rats underwent surgery on postnatal day 28 to allow intravenous (i.v.) selfadministration of nicotine or saline as described previously in Chapter 2. Rats were given ketoprofen (2-5 mg/kg, subcutaneous) and gentamicin $(5.5 \mathrm{mg} / \mathrm{kg}$, i.v.) on the day of surgery and for the 2 subsequent days. To assess and help maintain patency, the catheter was flushed every other day with heparinized saline prior to daily sessions. This method allows the experimenter to easily monitor patency of the catheter without significantly impairing rats during prolonged self-administration sessions.

\section{Nicotine self-administration}

The self-administration methods were described in detail previously in Chapter 3 (page 34). Briefly, rats were initially trained to self-administer nicotine (male, $\mathrm{n}=20$; female, $n=17$ ) or saline (male, $n=21$; female, $n=18)$ under restricted access conditions (20 infusions maximum/day) to reduce variability in intake prior to extended access selfadministration beginning on postnatal day 30 . These sessions were almost $24-\mathrm{hr}$ in 
duration (sessions began at 1200 and ended at 1150 the next day) and were conducted over 5 daily sessions beginning on postnatal day 30. At the beginning of each session, the active lever was presented into the chamber and responses on it were reinforced under a fixed ratio 1 schedule. Infusions were paired with illumination of the active lever light and the sound of the pump for the duration, and thus served as discrete cues associated with drug delivery. The active lever was retracted from the self-administration chamber at either 1150 or once a maximum of 20 infusions had been obtained. Acquisition of nicotine self-administration was defined as 2 consecutive days of 20 infusions with a 2:1 preference of the active lever over the inactive lever. A 10-day extended access selfadministration period began following the fifth training session (on postnatal day 35). There was no limit on number of infusions obtained during these extended access $23-\mathrm{hr}$ fixed ratio 1 sessions (sessions began at 1200). Access was again restricted on postnatal days 45 and 46 to 20 infusions/session to equate nicotine intake between groups prior to abstinence.

\section{Experiment 1:}

\section{Wheel running during abstinence}

After the last day of self-administration (postnatal day 47), rats were randomly assigned to either an unlocked wheel that could freely rotate (male: saline, $\mathrm{n}=11$; nicotine, $\mathrm{n}=10$; female: saline, $\mathrm{n}=9$; nicotine, $\mathrm{n}=9$ ) or a locked wheel that was stationary (male: saline, $\mathrm{n}=11$; nicotine, $\mathrm{n}=10$; female: saline, $\mathrm{n}=9$; nicotine, $\mathrm{n}=9$ ). A metal gate, that separated the polycarbonate cage from the wheel, regulated access to the 
wheel. The gate was manually lifted by the experimenter each day at 1000 and lowered following 2 hours (1200). A 2-hour exercise session was selected based on our previous results with males demonstrating that this level access during abstinence was sufficient to reduce subsequent nicotine-seeking (see Chapter 3; Sanchez et al. 2013a). During the 2hour sessions, rats were free to move between the wheel and their polycarbonate cage. Revolutions completed during each unlocked wheel session were recorded daily.

\section{Extinction/reinstatement}

Following the last wheel session, rats were placed back into their previous selfadministration chambers to allow for habituation prior to extinction/reinstatement testing. Testing began the next morning (postnatal day 57) under a within-session extinction/reinstatement paradigm using methods described previously in Chapter 3 (pg 36). Briefly, animals underwent a minimum of 5 extinction sessions, each lasting 1-hour and separated by a 5-minute timeout period, in which a response on the active lever had no programmed consequence. Most animals met the extinction criterion (i.e. fewer than 15 responses) within 5 sessions, although 5 out of 76 animals did require a $6^{\text {th }}$ session to extinguish responding. Following the last extinction session a 1-hour reinstatement session began with the presentation of the cues formerly associated with an infusion and all subsequent responses on the active lever resulted in the presentation of these cues. 


\section{Experiment 2:}

In order to examine the potential effects of the presence of the wheel itself on subsequent nicotine-seeking, we included additional groups of males $(n=8)$ and females $(n=8)$ housed in polycarbonate cages without access to running wheels during abstinence. These groups were included based on results from pilot studies demonstrating that in females, but not males, levels of responding during extinction and reinstatement were low in both wheel-condition groups. This finding was unexpected since females have been shown to exhibit higher levels of motivation to obtain nicotine infusions as compared to males (Donny et al. 2000), and acquire nicotine self-administration more readily than males (Lynch 2009). Females have also been found to display more robust drug-seeking behavior than males following abstinence from other drugs of abuse (Kerstetter et al. 2008). Thus, we hypothesized that females are sensitive to the attenuating effects provided by the presence of the wheel itself. To test this hypothesis, additional males and females were tested under the same self-administration procedure as described above in Experiment 1; however, following self-administration, they were moved to polycarbonate cages without wheel attachments for the 10-day abstinence period. Nicotine-seeking was assessed under the same within-session extinction/reinstatement paradigm as described above.

Data analysis

All data are presented as mean \pm standard error of mean (SEM). Data analysis for Experiment 1 was performed in locked and unlocked wheel groups for males and 
females. For Experiment 2 data analysis, the no wheel condition was compared to the locked and unlocked wheel conditions. Repeated measures analysis of variance (ANOVA) was used to analyze group and sex differences in extended access selfadministration (Fig 9), lever responses during extinction sessions (Fig 11), and to compare responding during the last extinction session to responding during the reinstatement session (Fig 13) with sex, drug, and wheel-condition as between subject factors and day or session as the within subject factors. Repeated measure ANOVA was also used to analyze wheel running during abstinence (Fig 10) and the average distance run during the first 3 days as compared to the last 3 days (Fig 11) with sex and drug as between subject factors and day as the within subject factor. Subsequent repeated measures ANOVAs within each drug condition (Fig 9 and 11) and sex (Fig 11 and 13) were used to further elucidate the sex difference in self-administration and extinction responding and the effect of wheel condition on extinction and reinstatement responding. Univariate analyses were used to analyze total responding during extinction (Fig 12), number of sessions to reach extinction criteria, and inactive lever responding during extinction and reinstatement with sex, drug, and wheel condition as between subject factors. Due to our predicted hypothesis that females would self-administer more nicotine than males, a one-tailed t-test was used to compare nicotine intake between male and female no wheel groups during extended access. Post-hoc comparisons were performed using one-tailed t-tests to compare total extinction responding between wheel groups based on our predicted hypothesis that the locked wheel condition and/or no wheel access would lead to greater nicotine-seeking than the unlocked wheel condition. Pearson 
correlations were used to determine associations between distance run during abstinence and nicotine intake, total extinction responding, and responding during reinstatement. Statistical analyses were performed using SPSS (version 20) and alpha was set to 0.05 for all tests.

\section{Results}

\section{Experiment 1}

Sex differences in nicotine self-administration

Under 23-hour extended access conditions, both male and female rats selfadministered significantly more nicotine infusions than saline infusions (overall effect of drug, $F_{1,67}=71.21, p<0.001$; Fig. 9A). Although no differences were observed among saline controls, within nicotine groups there was a significant effect of $\operatorname{sex}\left(\mathrm{F}_{1,33}=7.650\right.$, $\mathrm{p}<0.05)$, but a non-significant trend for the effect of day $(\mathrm{p}=0.07)$ and no interaction of day by sex, indicating that while females took more nicotine than males, the pattern of intake across sessions was similar between the sexes particularly during the first 7 days of extended access. Interestingly, however, there was a notable decrease in responding for nicotine during the last 3 days of access (postnatal days 42-44) in the female group. Overall, no significant effect of wheel-condition was observed in nicotine or saline infusions (Fig. 9B) indicating that prior to abstinence and subsequent extinction/reinstatement testing, intake was similar between the locked and unlocked wheel groups. 

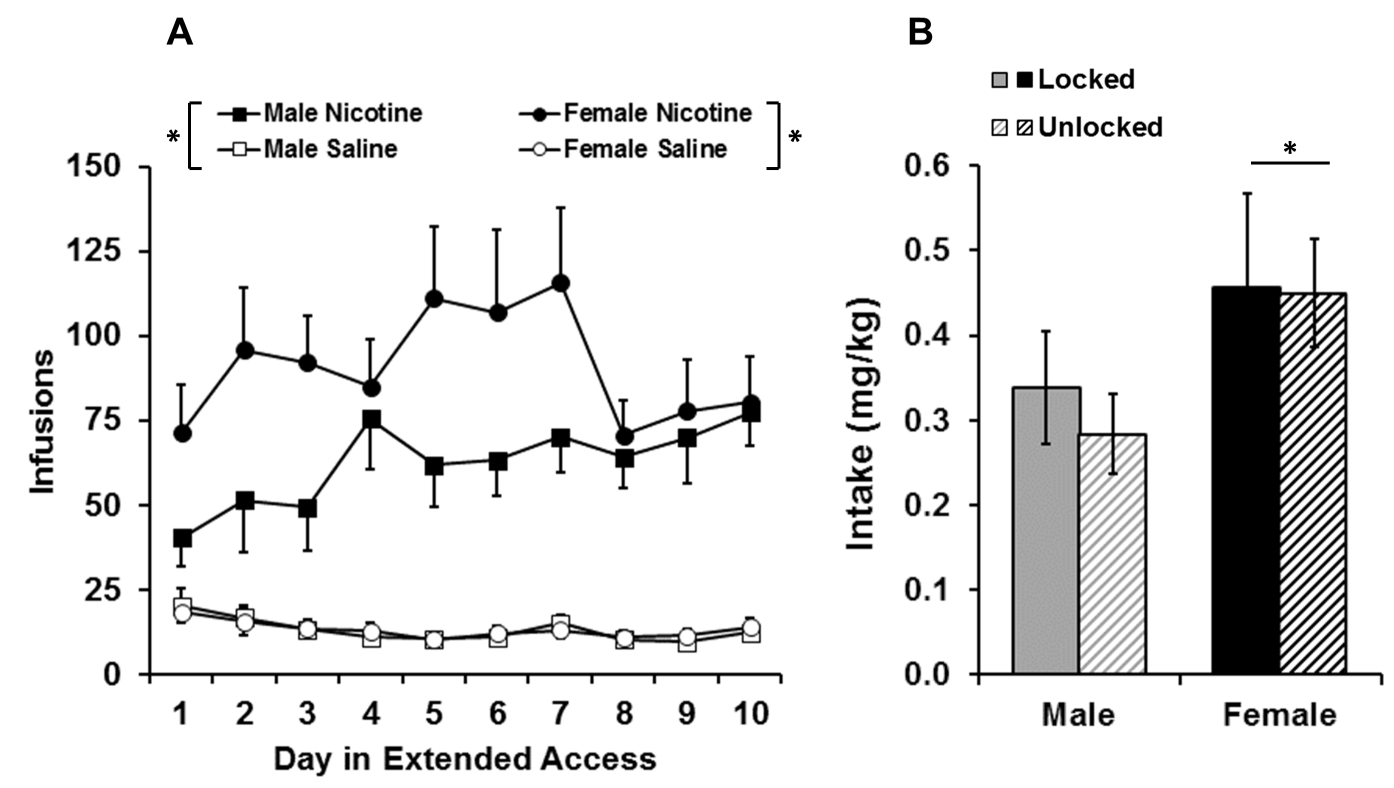

Figure 9. Adolescent females self-administer more nicotine than males under extended access conditions. (A) Average (mean \pm SEM) number of infusions self-administered during 10-day extended access sessions for nicotine (black symbols) and saline (white symbols) in male (square symbols) and female (circle symbols) groups. Asterisk indicates significant $(*, \mathrm{p}<0.05)$ difference in number of infusions between nicotine (male, $\mathrm{n}=$ 20; female, $n=17$ ) and saline (male, $n=21$; female, $n=18$ ) groups. (B) Average daily nicotine intake (mean \pm SEM) for male (locked, $n=10$; unlocked $n=10$ ) and female (locked, $\mathrm{n}=8$; unlocked, $\mathrm{n}=9$ ) groups. Asterisk indicates significant difference in average daily intake between males and females $(*, p<0.05)$. 
Sex differences in wheel running during abstinence

Female rats ran more than their male counterparts during the daily 2-hour sessions (overall effect of sex, $F_{1,35}=40.35, p<0.001$; Fig. 10). We also observed significant effects of day $\left(\mathrm{F}_{9,315}=8.73, \mathrm{p}<0.001\right)$ and day by $\operatorname{sex}\left(\mathrm{F}_{9,315}=3.14, \mathrm{p}<0.01\right)$ indicating that wheel running increased differently between males and females over time. While analysis within each sex revealed a significant effect of day for both males and females, this effect in males appeared to be due to a difference from the first day to the last day rather than a consistent increase over time. In fact, when this day was excluded the day effect was no longer significant in males but was retained in females. Furthermore, an analysis of the first 3 days to the last 3 days revealed significant effects of $\operatorname{sex}\left(\mathrm{F}_{1,35}=\right.$ 44.20, $\mathrm{p}<0.001)$, day $\left(\mathrm{F}_{1,35}=17.90, \mathrm{p}<0.001\right)$, and day by $\operatorname{sex}\left(\mathrm{F}_{1,35}=7.70, \mathrm{p}<0.01\right)$ indicating that females increased their running $\left(\mathrm{F}_{1,16}=15.15, \mathrm{p}<0.01\right)$ while males did not. Although levels of wheel running appeared higher in the nicotine groups as compared to saline groups, particularly within females, the level of variability was relatively high, and no significant effects of drug or drug by sex were observed. No correlation was found between distance run during abstinence and infusions during extended access. 


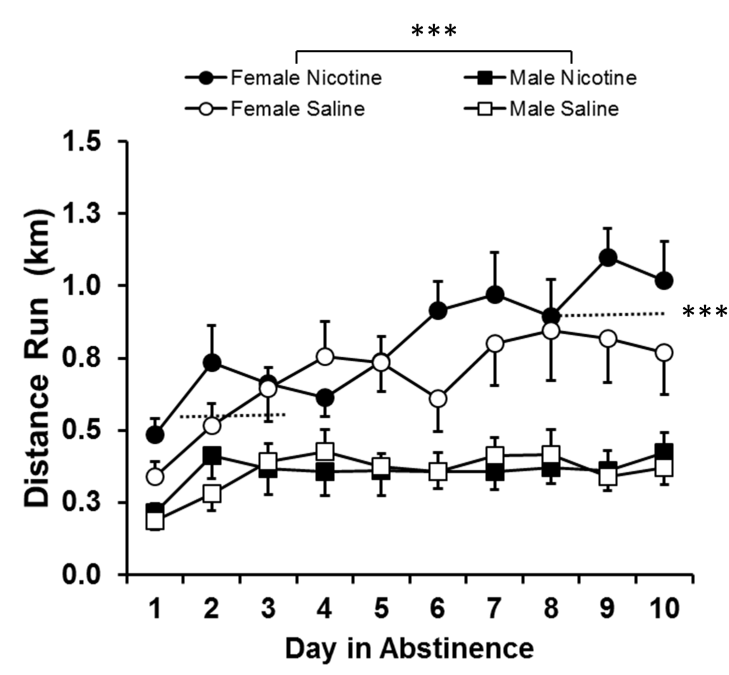

Figure 10 Females run further and escalate their running to a greater extent than males. Average (mean \pm SEM) daily distance run during abstinence for females (saline, $\mathrm{n}=9$; nicotine, $\mathrm{n}=9$ ) and males (saline, $\mathrm{n}=11$; nicotine, $\mathrm{n}=10$ ) in the unlocked wheel condition is plotted. Asterisks indicate significant difference in distance run between males and females and significant increase in average daily distance run during the last 3 days as compared to the first 3 days in females $(* * *, p<0.001)$.

Sex differences in the effect of wheel running during abstinence on subsequent nicotineseeking during extinction and reinstatement

Prior nicotine self-administration led to significantly more active lever responding during extinction sessions than did prior saline self-administration (effect of drug, $F_{1,68}=48.08, p<0.001 ;$ Fig. 11$)$ indicating that adolescent-onset nicotine selfadministration led to robust drug-seeking. Within the saline controls, there was a significant effect of session $\left(\mathrm{F}_{4,140}=3.53, \mathrm{p}<0.01\right)$, with the greatest number of responses in the first session as compared to later sessions. There was also a trend for an 
effect of sex $(p=0.058)$, with females tending to have higher levels of responding as compared to males. Within the nicotine animals, higher responding was again observed during the first session as compared to later sessions (effect of session, $\mathrm{F}_{4,132}=7.58, \mathrm{p}<$ 0.001), but there was a similar pattern of responding in males and females. Although an overall effect of sex was not found in the nicotine animals, a significant sex by wheel condition was observed $\left(\mathrm{F}_{1,33}=5.414, \mathrm{p}<0.05\right)$. Within the nicotine males there was a significant effect of wheel condition $\left(F_{1,18}=9.21, p<0.01\right)$ with those in the locked wheel condition displaying higher levels of responding than those in the unlocked wheel condition (Figs 11 and 12). However, in the nicotine females, there was no difference in responses between the wheel conditions; both groups showed low levels of responding which were statistically similar to males in the wheel condition. There were no associations between the distance run during abstinence and the number of active lever responses during extinction. Inactive lever responding during extinction sessions was significantly higher in nicotine groups $\left(\mathrm{F}_{1,68}=15.92, \mathrm{p}<0.001\right)$ as compared to saline groups; however, no effects of sex or wheel-condition were observed. Inactive lever responding in saline males under the locked and unlocked wheel condition was $6.00 \pm$ 3.05 and $6.27 \pm 3.07$, respectively, and in saline females in these same conditions was 2.5 \pm 1.15 and $4.67 \pm 2.09$. Inactive responding in nicotine males under the locked and unlocked wheel condition was $15.9 \pm 3.14$ and $13.7 \pm 4.59$, respectively, and in nicotine females in these same conditions was $14.5 \pm 3.84$ and $8.33 \pm 2.30$.

Nicotine animals also took significantly longer to extinguish responding as compared to saline controls $\left(\mathrm{F}_{1,68}=19.615, \mathrm{p}<0.001\right)$. Specifically, the average number 
of sessions to reach the extinction criterion (i.e. fewer than 15 active lever responses) for nicotine males in the locked and unlocked wheel condition was $3.67 \pm 0.62$ and $3.13 \pm$ 0.74 , respectively as compared to $1.3 \pm 0.24$ and $1.55 \pm 0.44$ for saline males in these same conditions. The average number of sessions to reach the extinction criterion for nicotine females was $3.00 \pm 0.74$ and $2.22 \pm 0.28$ in the locked and unlocked wheel conditions, respectively as compared to $1.67 \pm 0.33$ and $1.56 \pm 0.44$ for saline females in these same conditions. No effects of wheel condition or sex were observed on rates of extinction. However, the average number of extinction sessions run did not differ between groups as the majority of animals (71 out of 76) in all groups met the extinction criterion by the $5^{\text {th }}$ session. 


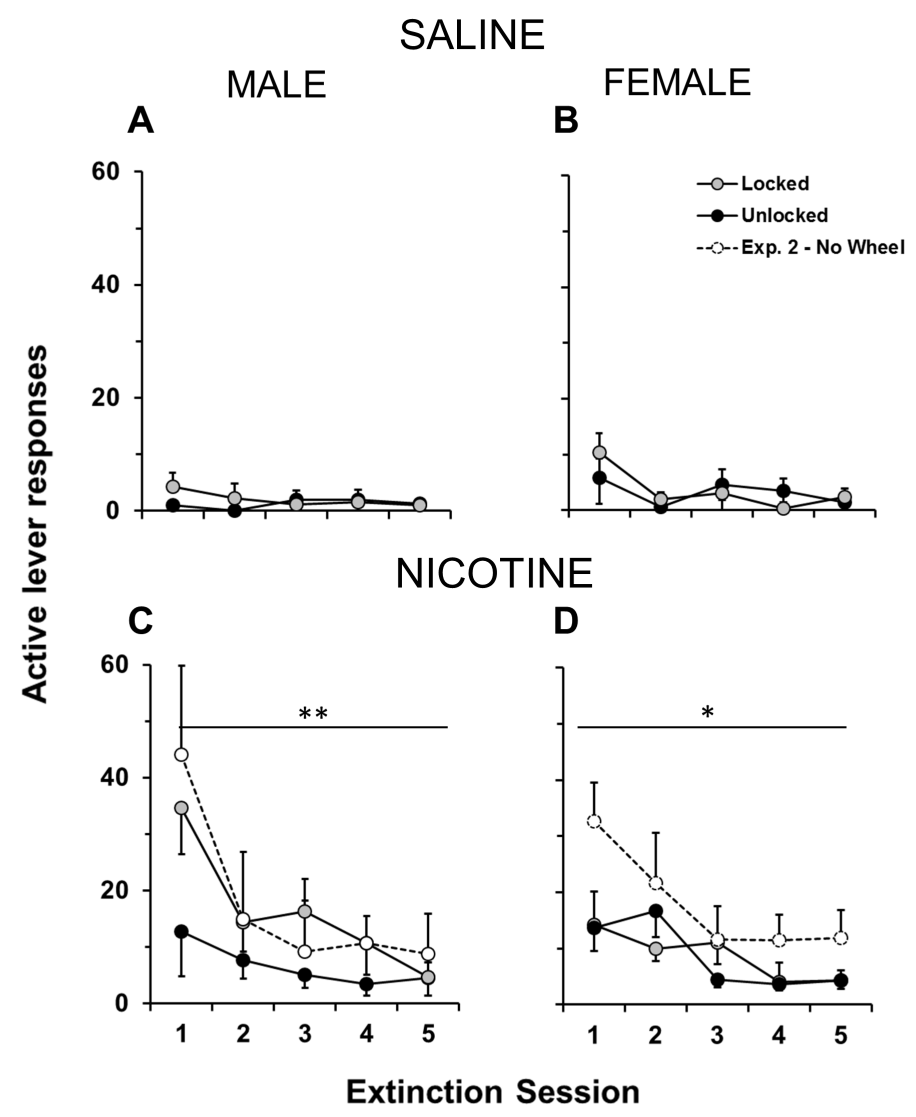

Figure 11. Nicotine self-administration during adolescence followed by an abstinence period leads to robust nicotine-seeking during extinction. Average active lever responses during each of the 5 extinction sessions are plotted for males (saline, A; nicotine, $\mathrm{C}$ ) and females (saline, B; nicotine, D) and for the locked (gray circles) and unlocked (black circles) wheel conditions. In panels $\mathrm{C}$ and $\mathrm{D}$, the extinction responding for the no wheel groups (white circles) from experiment 2 are also plotted for comparison. Asterisks indicate significantly more active lever responding during extinction in nicotine males (C) and females (D) as compared to saline controls (A and B, respectively; ${ }^{* *}, \mathrm{p}<0.01$; $*, \mathrm{p}<0.01)$. 


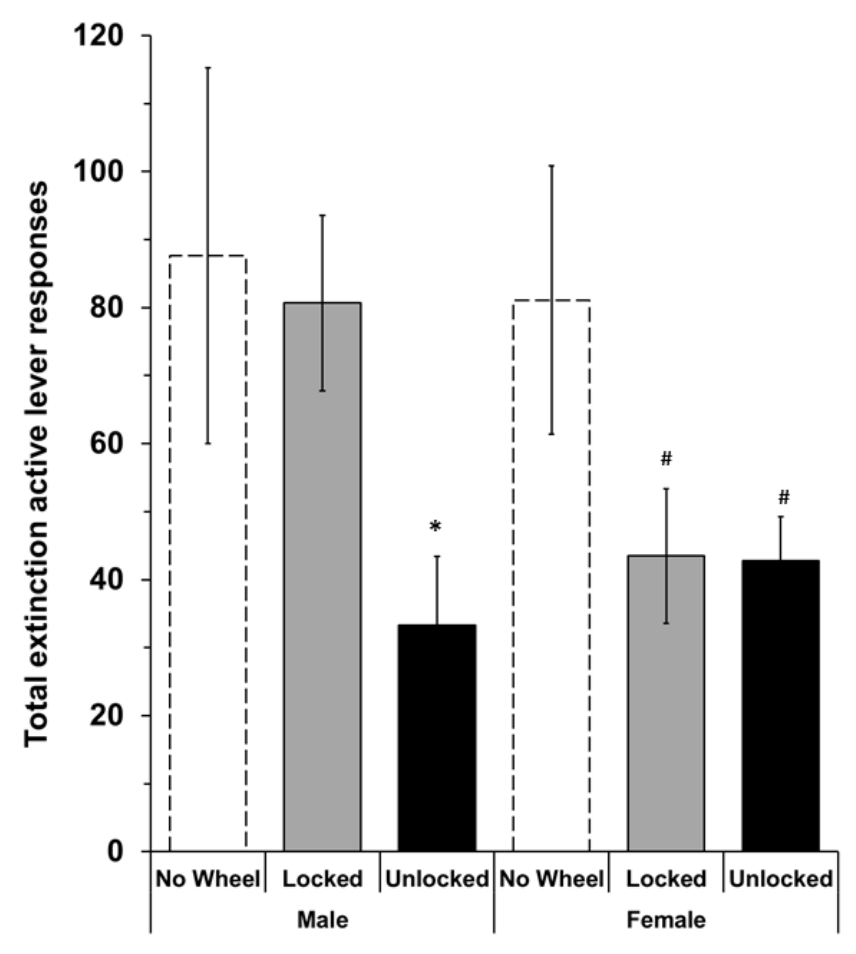

Figure 12. Total active lever responses (mean \pm SEM) during the first 5 extinction sessions for males $(\mathrm{n}=8, \mathrm{n}=10, \mathrm{n}=10)$ and females $(\mathrm{n}=8, \mathrm{n}=8, \mathrm{n}=9)$ for the no wheel, locked, and unlocked groups are displayed. Asterisk indicates significant difference from no wheel and locked wheel groups in males $(*, p<0.05)$ and number sign indicates significant difference from no wheel group in females $(\#, p<0.05)$.

Prior nicotine self-administration also led to significantly higher levels of cueinduced reinstatement than did saline self-administration (overall effect of drug, $\mathrm{F}_{1,68}=$ 23.52, $\mathrm{p}<0.001)$. Within nicotine animals, there was a significant session effect $\left(\mathrm{F}_{1,33}=\right.$ $27.14, \mathrm{p}<0.001)$ with all groups significantly increasing their responding during reinstatement from the last extinction session; however, no effect of wheel condition or sex was observed. Within saline controls, a sex difference was observed due to higher 
levels of reinstatement responding within females (effect of sex, $F_{1,35}=5.75, p<0.05$ ) but only a trend for a difference was observed between the last extinction session and the reinstatement session, and again there was no effect of wheel condition. There were no associations between distance run during abstinence and active lever responses during reinstatement. Similar to inactive lever responding during extinction, inactive lever responding during reinstatement was significantly higher in nicotine as compared to saline groups $\left(\mathrm{F}_{1,68}=7.65, \mathrm{p}<0.01\right)$ and there were no effects of sex or wheel-condition. Inactive lever responding during reinstatement in saline males were $0 \pm 0$ and $1.82 \pm 0.96$ and in females $0.44 \pm 0.34$ and $0.78 \pm 0.57$ in the locked and unlocked wheel groups, respectively. Inactive lever responding during reinstatement in nicotine males were $1.1 \pm$ 0.64 and $2.9 \pm 1.91$ and in females were $4.38 \pm 1.46$ and $4.44 \pm 2.68$ in the locked and unlocked wheel groups, respectively.

Initially, weights were similar among males and females $(88.9 \mathrm{~g} \pm 1.7$ versus 84.9 $\mathrm{g} \pm 1.3$ at the start of the experiment, respectively); however, by the end of the experiment males weighed significantly more than females $(285.5 \mathrm{~g} \pm 5.1$ versus $212.4 \mathrm{~g}$ \pm 3.2 , respectively; sex effect $F_{1,57}=150.789, \mathrm{p}<0.001$ ). No effect of wheel condition was observed for either sex, but by the end of the experiment, saline males weighed significantly more than nicotine males $(297.8 \pm 5.9 \mathrm{~g}$ versus $276.4 \pm 7.2 \mathrm{~g}$, respectively; drug effect, $\left.F_{1,28}=7.468, \mathrm{p}<0.05\right)$. 


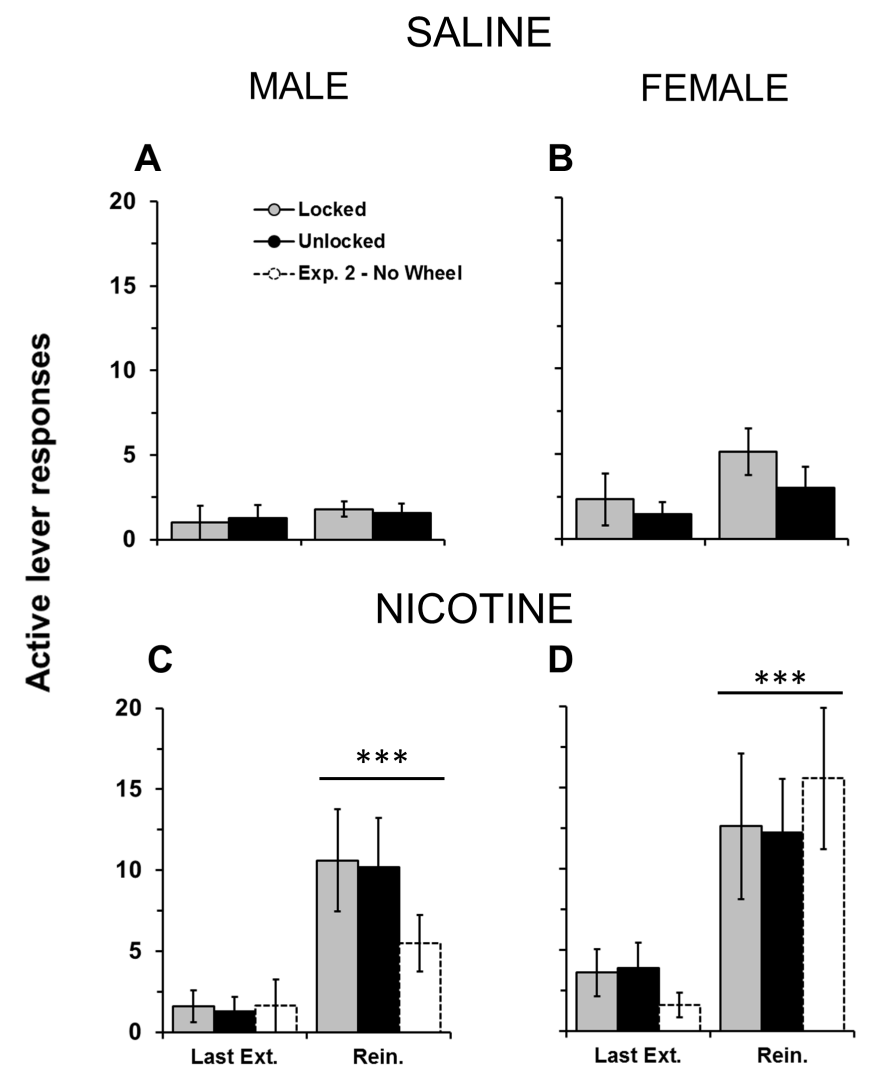

Figure 13 Total active lever responses (mean \pm SEM) during the last extinction session (Last Ext.) and reinstatement session (Rein.) are plotted for saline males (A) and females (B) and nicotine males (C) and females (D). Locked, unlocked, and no wheel

(Experiment 2) groups are portrayed by gray, black, and white bars, respectively.

Asterisk indicates a significantly greater responding during reinstatement as compared to the last extinction session $(* * *, p<0.001)$. 


\section{Experiment 2}

Nicotine-seeking during extinction is attenuated by both wheel conditions in females but not males

Prior to abstinence, males and females without access to a running wheel during abstinence (no wheel) did not differ from their locked and unlocked wheel counterparts in nicotine intake during extended access (males, $0.26 \pm 0.05 \mathrm{mg} / \mathrm{kg}$; females, $0.47 \pm 0.08$ $\mathrm{mg} / \mathrm{kg})$ and again a significant effect of sex was observed $\left(\mathrm{F}_{1,47}=8.385, \mathrm{p}<0.01\right)$. Average daily intake was significantly greater in females in the no wheel condition as compared to males $\left(\mathrm{t}_{14}=4.854, \mathrm{p}<0.05\right)$. A significant effect of wheel condition was observed in total extinction responding $\left(\mathrm{F}_{1,47}=5.317, \mathrm{p}<0.01 ;\right.$ Fig 12$)$. Although significant effects of sex and sex by wheel condition were not observed overall, planned analysis within each sex revealed interesting differences in the effects of wheel condition. Within males, a significant effect of wheel condition was again observed for total active lever responding during extinction sessions $\left(\mathrm{F}_{2,25}=6.418, \mathrm{p}<0.05\right)$. Males in the no wheel condition did not differ significantly from those in the locked wheel condition in active lever responding during extinction and they displayed significantly greater responding than males in the locked wheel condition $\left(\mathrm{t}_{16}=4.112, \mathrm{p}<0.05\right)$. The females in the no wheel group did, however, show significantly elevated nicotine-seeking during extinction sessions (effect of wheel condition, $F_{2,22}=3.88, p<0.05$; Fig 12 ) as compared to the locked or unlocked wheel condition female groups (locked versus no wheel $\mathrm{t}_{14}=$ 3.968, $\mathrm{p}<0.05$; unlocked versus no wheel, $\mathrm{t}_{15}=4.482, \mathrm{p}<0.05$; Fig 12). In fact, levels of extinction responding were not different from those observed in males that were in the 
locked wheel condition during abstinence. As in Experiment 1, a significant increase in responding was observed in the reinstatement session as compared to the last extinction session (session effect, $\mathrm{F}_{1,47}=37.240, \mathrm{p}<0.001$ ) and no significant effect of wheel condition was observed. However, with the addition of the no wheel groups, a significant effect of sex emerged $\left(\mathrm{F}_{1,47}=4.633, \mathrm{p}<0.05\right)$ due to higher levels of reinstatement in the no wheel females compared to the no wheel males $\left(\mathrm{t}_{14}=2.177, \mathrm{p}<0.05\right)$.

\section{Discussion}

The goal of the present study was to determine if there are sex differences in the effect of wheel running during abstinence on subsequent nicotine-seeking. As was anticipated based on our previous findings in Chapter 3 (Sanchez et al. 2013a), we observed robust levels of nicotine-seeking during extinction in males under the locked and no wheel conditions, and these levels were markedly reduced by running under the unlocked wheel condition. Surprisingly, in females, levels of nicotine-seeking during extinction were low under both wheel conditions, locked and unlocked, with levels comparable to those observed in males tested under the unlocked wheel condition. Females in the no wheel condition, however, showed high levels of nicotine-seeking during extinction that were similar to males without access to wheel or with a locked wheel access. Levels of nicotine-seeking in these females were also higher than either of the female wheel groups. Thus, while wheel running during abstinence effectively reduced subsequent nicotine-seeking in males, the presence of the wheel was sufficient to reduce nicotine-seeking in females. 
Although environmental enrichment is one possible mechanism to account for the lower levels of nicotine-seeking during extinction in females under both wheel conditions (Chauvet et al. 2009), another possibility is that the locked wheel condition permits physical activity within the wheel and thus also functions as an exercise condition. While the locked wheel condition is an important control for environmental enrichment, animals are still able to be quite active within the wheel (e.g., climbing and hanging; Koteja et al. 1999). Females have been shown to be more active than males (Hitchcock 1925; Elkelboom and Mills 1988; Smith et al. 2011, 2012), and here we also showed that females ran greater distances in the unlocked wheel condition as compared to males. As such, females may have been more active in the locked wheel as well and thus may have been more sensitive to this alternative form of exercise within the wheel as compared to males. This finding may also suggest that non-aerobic exercise could benefit females but not males. Further studies using photocells or video tracking to directly monitor activity within wheels are needed to dissociate the effects of environmental enrichment versus exercise on nicotine-seeking in females. It is also possible that the sucrose active lever training used in this study differentially affected subsequent active lever responding for nicotine between males and females. Further research investigating the impact of sucrose pre-training on subsequent drug-seeking in adolescents and potential sex differences for this effect is needed.

Consistent with our hypothesis, adolescent females self-administered significantly more nicotine under 23-hour unlimited access conditions as compared to males. Although the present study is the first preclinical study to demonstrate sex differences in nicotine 
self-administration under extended access conditions, previous work from our lab has demonstrated an increased sensitivity to the positive reinforcing effects of nicotine in adolescent females with higher rates of initiation and levels of motivation to selfadminister nicotine as compared to adolescent males under the same low dose condition used in the present study (Lynch 2009). Interestingly, this increased motivation was found beginning on postnatal day 43 under a progressive ratio schedule and in the present study we found that as a group, females decreased the number of responses for nicotine under a fixed ratio schedule during the last 3 days of self-administration corresponding to postnatal days $42-44$. Thus, the decrease in responding observed here might have been due to an increased sensitivity to the positive reinforcing effects of nicotine at this later stage of adolescence. Further research is needed to directly examine this possibility. Similar to findings in adolescents, adult female rats have also been shown to acquire nicotine self-administration faster (at low doses), achieve higher breakpoints under progressive ratio conditions, and have shorter latencies to first response during both fixed ratio and progressive ratio conditions as compared to males (Donny et al. 2000). Nonetheless, some studies have failed to find a sex difference in adolescent and adult nicotine self-administration under fixed ratio conditions (Chen et al. 2007; Levin et al. 2011; Feltenstein et al. 2012; Li et al. 2012). However, these studies used short-access paradigms (1-2 hours/session) and/or higher doses of nicotine than used in the present study, which may account for the discrepancy with our findings. Notably, Chen et al. (2007) used the same extended access paradigm in adolescent rats and failed to observe sex differences likely due to the use of higher nicotine doses (i.e. 7.5, 15, 30, and 60 
$\mu \mathrm{g} / \mathrm{kg}$ ), which may obscure individual differences (Donny et al. 2000, Lynch 2006, 2009). Li et al. (2012) did, however, find that under progressive ratio conditions adolescent female rats responded significantly more than adolescent male rats. Also, Levin et al. (2011) found that although females did not self-administer more initially, their responding for nicotine persisted longer than that of males. Thus, it appears that adolescent and adult females may be more vulnerable than males to the positive reinforcing effects of nicotine.

In contrast to our hypothesis, females did not display greater nicotine-seeking following a prolonged abstinence period as compared to males. Psychostimulant drugs such as nicotine and cocaine are known to incubate over an abstinence period (i.e. drugseeking increases with longer abstinence periods; Grimm et al. 2001; Lu et al. 2004;

Abdolahi et al. 2010) and there is evidence that there are sex differences in this incubation effect for cocaine with females displaying greater levels of cocaine-seeking than males following prolonged abstinence (Kerstetter et al. 2008). Although, no studies have directly examined sex differences in the incubation effect of nicotine-seeking, our findings here as well as recent findings from others suggests that sex differences in drugseeking following a prolonged drug abstinence period do not extend to nicotine (Feltenstein et al. 2012).

Also in contrast to our hypothesis, we did not find an effect of exercise on cueinduced reinstatement in either males or females. Although nicotine self-administration followed by prolonged abstinence did lead to a significant increase in responding during cue-induced reinstatement, this effect was modest and, as was reported previously in 
males (Sanchez et al. 2013a), highly variable. The modest levels of cue-induced nicotineseeking observed here are likely due to the age of the animals in this study. Compared to adults, adolescent rodents show attenuated neurochemical adaptations during withdrawal from nicotine (Natividad et al. 2010, 2012; Tejeda et al. 2012). Adolescent animals also do not have complete development of critical brain regions, such as the prefrontal cortex (Geidd 2004; Counotte et al. 2011), that are known to be important for cue-induced reinstatement (Koya et al. 2008). Others have similarly reported modest levels of cueinduced cocaine-seeking in adolescent rats as compared to adults and other types of reinstatement within adolescents (i.e. stressor led or cocaine primed; Anker and Carroll 2010). Thus, the low level of reinstatement observed here in this adolescent-onset model of nicotine addiction may have created a floor effect for which the effects of exercise could not be determined. Further studies are needed to determine if other reinstatement paradigms (e.g. nicotine or stress induced reinstatement) would be more robust and thus a better tool to examine the effects of exercise.

Our findings are consistent with previous reports that demonstrate a significant attenuation of drug-seeking when wheel-running was available concurrently with cocaine extinction and reinstatement procedures (Zlebnik et al. 2010) or during abstinence from cocaine or nicotine prior to extinction and reinstatement (Lynch et al. 2010; Sanchez et al. 2013a). Although in humans, the data are equivocal for its long-term efficacy (Ussher et al. 2012), studies suggest that exercise may be an effective therapeutic for smoking cessation. Two recent meta-analyses of the literature found that exercise acutely attenuates the desire to smoke as well as tobacco withdrawal symptoms (Roberts et al. 
2012; Haasova et al. 2013). There is also some evidence that the effects of exercise extend to adolescents with results from a few studies demonstrating that the addition of an exercise component to behavioral treatment programs effectively reduces rates of smoking in adolescents (Werch et al. 2005; Horn et al. 2011; Horn et al. 2013). In an interesting parallel to our rodent studies, girls benefitted from after-school enrichment programs whereas boys only showed a reduction in smoking behavior when the program had an exercise component (Horn et al. 2011). Our preclinical findings suggest that these differences may be due in part to biological mechanisms.

In summary, wheel running during abstinence attenuated subsequent nicotine-seeking in male and female rats that began self-administration during adolescence. Given that most adult smokers initiate use during adolescence our results support the notion that exercise may be an effective smoking cessation aid in this vulnerable population. Sex differences were observed in sensitivity to the locked wheel condition either by functioning as an environmental enrichment or an alternative form of exercise. Thus, future studies in humans considering the use of exercise as a treatment for human smoking cessation, should explore the use of aerobic versus non-aerobic exercise with varying intensities in males and females. 


\section{Chapter 5: \\ Structural plasticity associated with exercise as an intervention for adolescent-onset nicotine addiction}

\section{Introduction}

Drugs of abuse including nicotine, cocaine, and amphetamine are known to have long-lasting effects on the structure of dendrites and dendritic spine density in key areas of the mesolimbic reward pathway (for review see, Robinson and Kolb 2004; Russo et al. 2010). For example, chronic intermittent exposure to non-contingent administration of nicotine followed by an abstinence period has been shown to increase dendrite length and dendritic spine density in the medial prefrontal cortex and nucleus accumbens shell in adult and adolescent male rats (Brown and Kolb 2001; McDonald et al. 2005; 2007). Only one study has examined structural plasticity following abstinence from selfadministered nicotine (Gipson et al. 2013). In this study performed in adult rats, they observed an increase in spine head diameter within the core region (Gipson et al. 2013). However, structural plasticity following abstinence from adolescent nicotine selfadministration has yet to be examined in either the core or the shell region of the nucleus accumbens. Furthermore, a direct comparison of structural plasticity within the core and shell regions remains to be investigated. Thus, one goal of this study was to determine if adolescent-onset nicotine self-administration followed by a prolonged abstinence period and subsequent nicotine-seeking, induces long-term plastic changes within the nucleus accumbens core and shell regions. 
Abstinence from drugs of abuse including nicotine is associated with long-lasting behavioral effects such as a progressive increase in vulnerability to relapse over a protracted period of abstinence (i.e., "incubation of craving"; Pickens et al. 2011; Abdolahi et al. 2010; Bedi et al. 2011). Abstinence from nicotine self-administration has been shown to induce nicotine-seeking in extinction and reinstatement procedures (Abdolahi et al. 2010; Sanchez et al. 2013a; b), an effect that has been associated with structural plasticity within the nucleus accumbens core in adult rats (Gipson et al. 2013). In previous chapters of this dissertation, voluntary wheel running exercise during abstinence was shown to attenuate subsequent nicotine-seeking in an adolescent-onset model (Sanchez et al.2013). Although the effect of exercise on neuronal structural plasticity within the nucleus accumbens is not known, exercise has been found to be rewarding and to affect dopamine signaling in the nucleus accumbens core and shell regions (Greenwood et al. 2011) as well as glutamate signaling in other regions of the brain (Meeusen et al. 1997; Guezennec et al. 1998). Furthermore, exercise has been found to increase dendrite length and spine density within the hippocampus (Stranahan et al. 2007; Eadie at al. 2005; Redila and Christie 2006), suggesting that exercise can modulate structural plasticity of dendrites. Thus, the second goal of this study was to determine if voluntary wheel-running exercise, that has previously been shown to attenuate nicotine-seeking, also attenuates nicotine-induced structural plasticity within the nucleus accumbens.

To address the goals of this study, rats were given voluntary access during a 10day abstinence period to either an unlocked (exercise) or a locked (sedentary) running 
wheel and then tested for nicotine-seeking under an extinction and reinstatement paradigm. To assess the structural plasticity associated with nicotine-seeking and its modulation by exercise, histological examination of synapses within the nucleus accumbens core and shell regions were performed using electron microscopy. We focused specifically on asymmetric synapses onto dendritic spines as these are the most common type of synapses found on spine heads and are widely accepted as excitatory synapses (Nimchinsky et al. 2002; Gray 1959). Furthermore, abstinence from nicotine has been shown to induce increases in spine head diameter and glutamate signaling within the nucleus accumbens (Gipson et al. 2013; Russo et al. 2010). It was hypothesized that increased nicotine-seeking following abstinence would result in an increase in synapse length in the nucleus accumbens core and shell regions and that exercise during abstinence would attenuate this effect.

\section{Methods}

Animals

Adolescent male Sprague-Dawley (Charles River Laboratories, Portage, ME, USA) rats $(\mathrm{N}=14)$ arrived on postnatal day 22 and were singly housed in selfadministration chambers (see Chapter 2 for details) under a 12-hour light/dark cycle (lights on 0700). Food and water were available ad libitum throughout the experiment. The health of the animals was monitored daily and they were weighed every Monday, Wednesday, and Friday. All procedures were in accordance with NIH guidelines and approved by the University of Virginia Animal Care and Use Committee. 
Drug

Nicotine bitartrate (Sigma-Aldrich, St. Louis, MO, USA) was dissolved in $0.9 \%$ sterile saline ( $\mathrm{pH} 7.4)$ and passed through a microfilter. A single low dose $(5 \mu \mathrm{g} / \mathrm{kg})$ of nicotine was selected based on previous work demonstrating that under the same experimental parameters robust acquisition and maintenance of self-administration as well as subsequent nicotine-seeking following abstinence were observed. The dose is expressed as the free base weight and infusions were delivered at a rate of $0.1 \mathrm{ml} / \mathrm{sec}$ based on the animal's weight. Nicotine solution was stored at $4{ }^{\circ} \mathrm{C}$ but was available at room temperature during self-administration sessions.

\section{Behavioral paradigm}

All behavioral methods (i.e. sucrose pre-training, surgery, nicotine selfadministration, abstinence, wheel-running, and extinction/reinstatement testing) were performed as described in Chapter 3. Briefly, prior to surgery, animals were food pretrained to respond on the active lever for sucrose pellets under an FR1 schedule until 2 days of 50 pellet deliveries. On postnatal day 28 , rats underwent surgery to implant a chronic indwelling catheter into the right jugular vein to allow intravenous nicotine $(\mathrm{n}=$ 8) or saline $(\mathrm{n}=6)$ self-administration. Self-administration training (5 days) began on postnatal day 30, under an FR1 schedule with a maximum of 20 infusions. Acquisition was defined as 2 consecutive days of 20 infusions with a 2:1 preference of the active lever over the inactive lever. The 10-day extended access self-administration phase began on postnatal day 35 during which animals could receive an unlimited number of infusions 
(FR1 schedule) during 23-hour sessions. Abstinence began after a 2-day equilibration phase during which infusions were again limited to 20/session. On the first day of abstinence (postnatal day 47), rats were moved from their self-administration chambers to a polycarbonate cage with either a locked (saline, $\mathrm{n}=3$; nicotine, $\mathrm{n}=4$ ) or unlocked wheel (saline, $\mathrm{n}=3$; nicotine, $\mathrm{n}=4$ ) attachment to live for the 10-day abstinence period. Each day, the experimenter removed a metal gate separating the polycarbonate cage from the wheel and rats were permitted to move freely between they cage and the wheel for 2 hours. Revolutions performed within the unlocked wheel were recorded daily. After the last wheel access session, rats were moved back to their self-administration boxes. The following morning, drug-seeking was tested under a within-session extinction and cueinduced reinstatement paradigm. During each of the 5 1-hour extinction sessions responses on the active lever had no programmed consequences. In this study, all animals met the extinction criterion (i.e. fewer than 15 responses in the last session) within 5 sessions. A 1-hour reinstatement session began following the last extinction session with the presentation of the cues formerly associated with a nicotine infusion (i.e. the light and the sound of the pump) and each subsequent response on the active lever resulted in the presentation of cues, however no infusions were delivered.

\section{Tissue preparation for electron microscopy}

Immediately following the end of the reinstatement session, rats were deeply anesthetized with a lethal dose of sodium pentobarbital and transcardially perfused with Tyrode's solution until the vasculature was clear ( $<2$ minutes) and then with a $4 \%$ 
paraformaldehyde with $0.5 \%$ gluteraldehyde fixative solution in $0.1 \mathrm{M}$ phosphate buffer (PB, pH 7.4) for 20-30 minutes. Brains were removed and placed in the same fixative solution overnight at $4{ }^{\circ} \mathrm{C}$. The following day, $80 \mu \mathrm{m}$ coronal sections including the nucleus accumbens were cut on a vibratome and stored in $0.1 \%$ sodium azide in PB at 4 ${ }^{\circ} \mathrm{C}$. Sections were washed in $0.1 \mathrm{M}$ PB and then postfixed in $1 \%$ osmium tetroxide in 0.1 M PB for 1-hour. Sections were subsequently washed with $0.1 \mathrm{M}$ PB three times (each wash 3 minutes) followed by $50 \%$ ethanol (ETOH) for 3-minutes and then stained with $4 \%$ uranyl acetate for 1 -hour. The sections were then serially dehydrated in $70 \%$ ETOH for 1 minutes, $90 \% \mathrm{ETOH}$ for 5 minutes, $100 \% \mathrm{ETOH}$ for 5 minutes twice, and acetone for 2 minutes 3 times. The last acetone wash was replaced with a 1:1 dilution of EPON in acetone in which the sections remained overnight. The following day the EPON-acetone mixture was replaced with EPON for 2-4 hours. Tissue was then transferred to an aclar sheet with EPON and covered with a another aclar and placed in an oven at $60{ }^{\circ} \mathrm{C}$ overnight. Camera lucida was used to trace landmarks of the tissue for localization of the nucleus accumbens core and shell regions. These regions were then cut from the flat embedded tissue and placed in a capsule BEEM that was subsequently filled with EPON and allowed to polymerize in the $60{ }^{\circ} \mathrm{C}$ oven for 3 days. Using a diamond knife, $70 \mathrm{~nm}$ sections of tissue were cut and collected on grids for visualization with a Joel 1010 transmission electron microscope (TEM). Images were captured using a digital camera. 
Quantitative analysis of electron micrographs

For each rat, 1 section was used to analyze the nucleus accumbens core and shell regions. Sections were selected at $1.92 \mathrm{~mm}$ Bregma based on a standard neuroanatomical atlas (Paxinos and Watson 2005). From each region (core and shell), 3 areas were selected parallel to the midline of the brain in the middle of the shell region and 3 corresponding areas were selected in the core region (blue boxes in Fig 14). Areas in the core were selected so that they were adjacent to areas that contained the anterior commissure (green boxes in Fig 14). Within each area 15 non-overlapping pictures were taken of neuropil at 10,000 times magnification with a 16M-pixel camera $(2.75 \mathrm{~nm}$ pixel size resolution, for a total of 45 pictures from each core and shell.

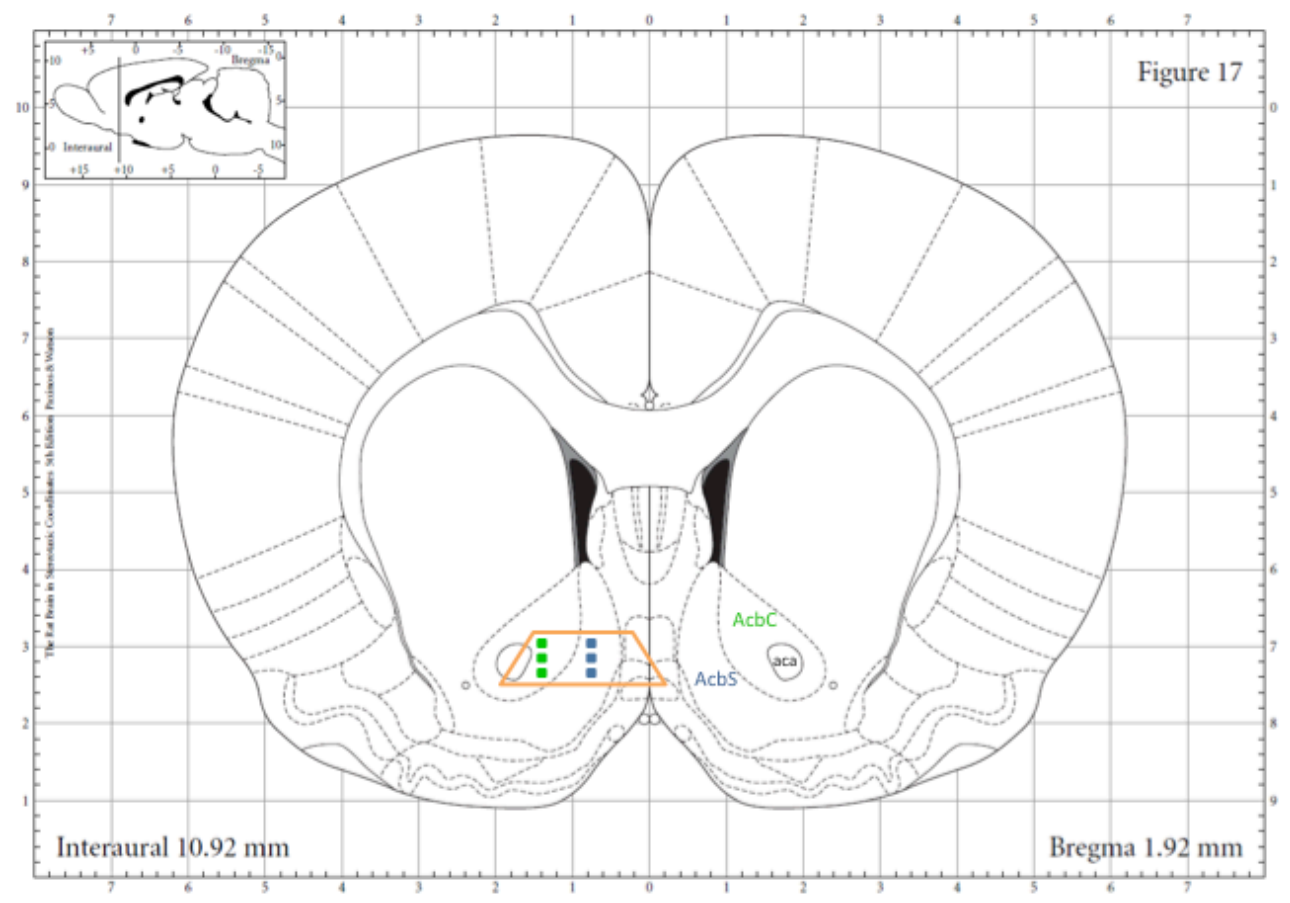

Figure 13 Schematic of areas within the nucleus accumbens core (AcbC; green boxes) and shell (AcbS; blue boxes) regions analyzed. The orange trapezoid represents the area from each section that used to make ultrathin sections. Not to scale. Modified from Paxinos and Watson 2005. 
Each photograph was examined by an experimenter blinded to the condition at a final resolution of 363 pixels/ $\mu \mathrm{m}$, and each synapse encountered was marked, its synapse length was measured. Total area of each photograph, excluding myelinated axons, blood vessels and cell bodies was also measured. Measurements of synapse length and neuropil area were taken using Image Pro Plus (Media Cybernetics, Silver Spring, MD). Synapses were defined by the presence of at least 3 synaptic vesicles in the presynaptic terminal and the parallel alignment of the pre- and post-synaptic membranes creating a synaptic cleft. Synapses were identified as either asymmetric (highlighted in blue in Fig 15) or symmetric (highlighted in green in Fig 15 Aii, Bii) based on the presence or absence of a postsynaptic density, respectively. Postsynaptic profiles were classified as spines if mitochondria and microtubules were absent (red arrows in Fig 15 Ai). The presence of a spine apparatus (black arrow in Fig 15 Ai) was also used for classifying postsynaptic profiles as a spine, but was not necessary. Other postsynaptic profiles were also identified as (1) dendritic shafts if microtubules and mitochondria were present (star in Fig 15 Ai) (2) cell bodies by the presence of endoplasmic reticulum, Golgi apparatus, or a nucleus or (3) an axon terminal if vesicles were present. 

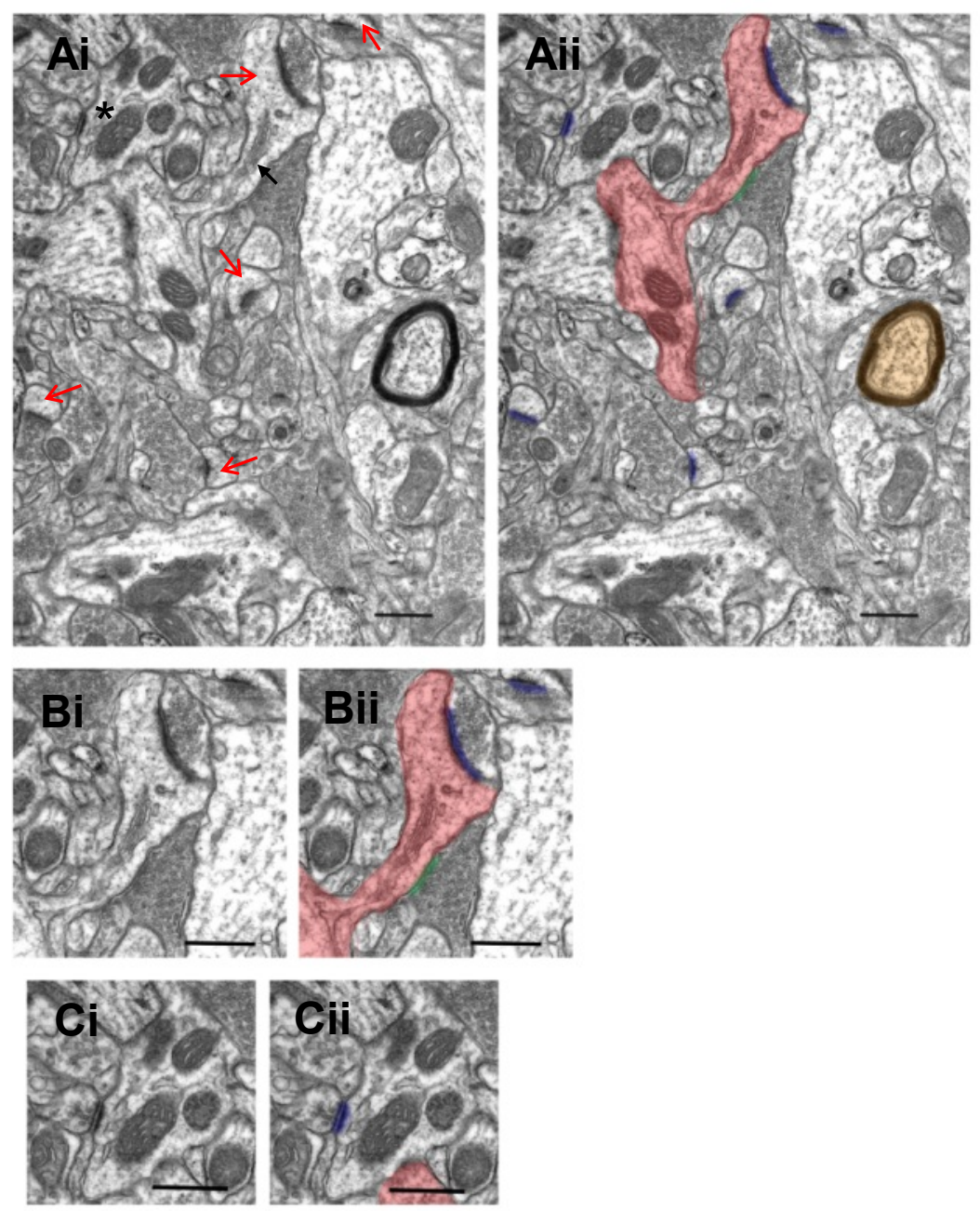

Figure 15 An example electron micrograph of neuropil from which synapses were measured. In panel Ai, red arrows mark dendritic spines, the black arrow marks a spine apparatus. In panel Aii, a dendritic spine that connects back to the dendritic shaft is highlighted in red, asymmetric synapses are highlighted in blue, symmetric synapse is highlighted in green, and a myelinated axon is highlighted in orange. Panels Bi and Bii show an enlarged image of a dendritic spine with asymmetric (blue) and symmetric (green) synapses. Panels $\mathbf{C i}$ and $\mathbf{C i i}$ show an enlarged image of an asymmetric synapse (blue) onto a dendrite with a clearly defined synaptic cleft. Scale bar represents $0.5 \mu \mathrm{m}$. 
Statistical analysis

Behavioral data are presented as the mean \pm SEM. Self-administration was analyzed using a repeated measures ANOVA with drug and exercise condition as the between subject factors and day as the within subject factor. T-tests were used to compare average daily intake between nicotine groups and average daily distance run between exercise groups. A univariate analysis as used to analyze differences in active lever responding during extinction and subsequent post hoc t-tests were performed. A repeated measures ANOVA of the last extinction session and the reinstatement session was used to determine differences in reinstatement of drug-seeking. Differences in the distribution of synapse length were analyzed using the Mann-Whitney U-test. The vast majority of synapses observed were in asymmetric synapses onto spines (approximately $90 \%$ ) thus all analyses were performed on measurements taken from this type of synapse. Statistical analyses were performed using SPSS (20) and alpha was set at 0.05 for behavioral data and 0.01 for histology data.

\section{Results}

\section{Behavioral results}

During extended access, animals that self-administered nicotine responded for significantly more infusions than those that self-administered saline with an overall effect of drug $\left(\mathrm{F}_{1,10}=10.825, \mathrm{p}<0.01\right.$; Fig 16A) and no effect of day. However, no significant effects of exercise condition or interaction of drug by exercise condition were observed. Furthermore, within the nicotine condition, no difference in nicotine intake was observed 
between the sedentary and exercise groups (Fig 16B). During abstinence, average daily distances run were similar between the saline and nicotine exercise groups (Fig 16C). During extinction, active lever responses were highest in the nicotine groups (effect of drug, $F_{1,10}=6.962, p<0.05 ;$ Fig 16D). A non-significant trend for a wheel by drug interaction was observed $(\mathrm{p}=0.062)$ and subsequent analyses revealed that the nicotine sedentary group had significantly higher responding as compared to both saline conditions (versus saline sedentary, $\mathrm{t}_{5}=4.366, \mathrm{p}<0.05$; versus saline exercise, $\mathrm{t}_{5}=$ 4.340, $\mathrm{p}<0.05)$ and the nicotine exercise group $\left(\mathrm{t}_{6}=3.996, \mathrm{p}<0.05\right)$. No significant difference in extinction responding was observed between the saline groups but the nicotine exercise group did have significantly higher responding than both the saline sedentary $\left(t_{5}=11.558, \mathrm{p}<0.001\right)$ and the saline exercise $\left(t_{5}=12.708, \mathrm{p}<0.001\right)$ groups. Reinstatement responding was low and not significantly higher than responding during last extinction session and no effect of drug or wheel condition was observed (Fig 16D). 
A

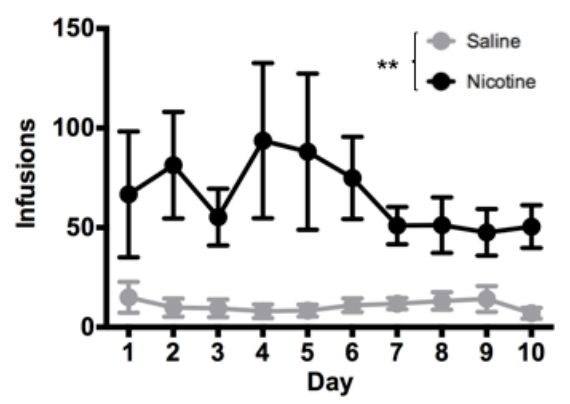

C

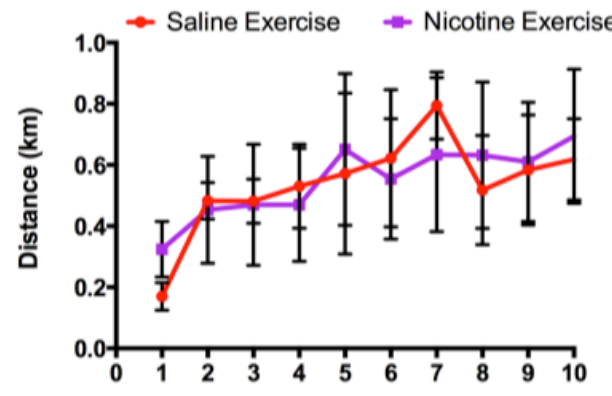

E

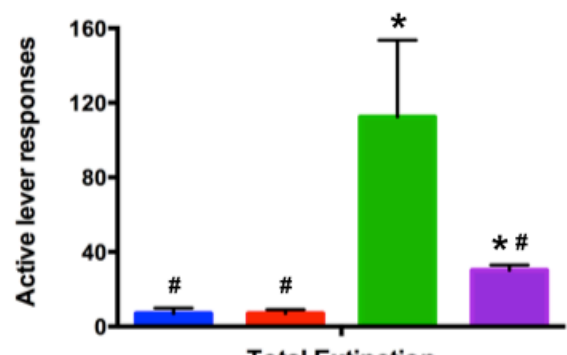

Total Extinction
B

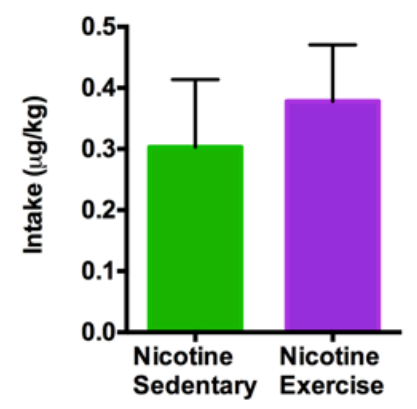

D

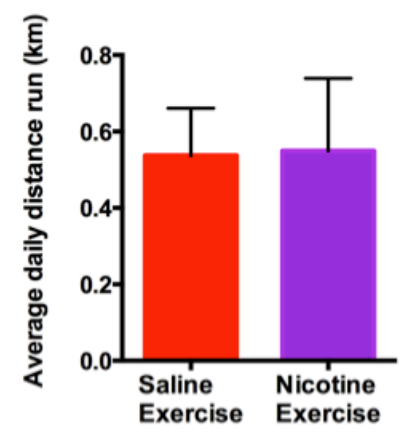

$\mathbf{F}$

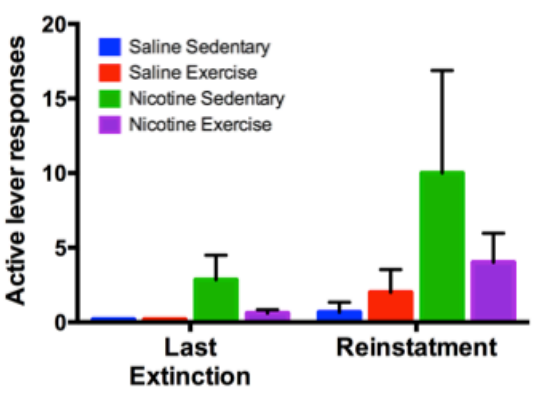

Figure 16 Behavioral data for extended access self-administration (A and B), distance traveled during abstinence $(\mathrm{C}$ and $\mathrm{D})$, and during extinction $(\mathrm{E})$ and reinstatement test $(\mathrm{F})$. In panel $\mathrm{A}$, the number of infusions is plotted for saline (gray circles; $\mathrm{n}=6$ ) and nicotine (black circles; $\mathrm{n}=8$ ) during the 10-day extended access self-administration period. Asterisks indicate a significant difference $(p<0.01)$ between saline and nicotine groups. In panel B, average daily nicotine intake is plotted for the nicotine sedentary (green bar; $n=4$ ) and exercise (magenta bar; $n=4$ ) groups. In panel $C$, the daily distance run in kilometers $(\mathrm{km})$ is plotted for each exercise session during the 10-day abstinence period. In panel $\mathrm{D}$, the average daily distance run is plotted for the saline (red bar; $\mathrm{n}=3$ ) and nicotine (magenta bar; $\mathrm{n}=4$ ) exercise groups. In panels $\mathrm{E}$ and $\mathrm{F}$, the active lever responses during all extinction sessions (Total Extinction; E), the last extinction session $(\mathrm{F})$, and the reinstatement session (F) are plotted for the saline sedentary (blue; $\mathrm{n}=3$ ), saline exercise (red), nicotine sedentary (green), and nicotine exercise (magenta) groups. Asterisks indicate a significant difference from saline sedentary group and the number signs indicate a significant difference from nicotine sedentary. 
Synapse length distribution in the nucleus accumbens core

Nicotine self-administration followed by abstinence (nicotine sedentary group) lead to a significant rightward shift in the distribution of synapse length as compared to the saline sedentary group $(\mathrm{p}<0.01 ;$ Fig $17 \mathrm{Ai}$ and Aii). The nicotine exercise group displayed a significant leftward shift in the distribution of synapse size as compared to the nicotine sedentary group ( $\mathrm{p}<0.001 ; \mathrm{Fig} 17 \mathrm{Bi}$ and $\mathrm{Bii})$. Indeed, the synapse length distribution of the nicotine exercise group did not differ from the saline sedentary group (Fig $17 \mathrm{Ci}$ and Cii). Thus, abstinence from nicotine self-administration led to increased synapse length while the exercise during abstinence normalized synapse length back to saline levels in the core region of adolescent rats.

Ai

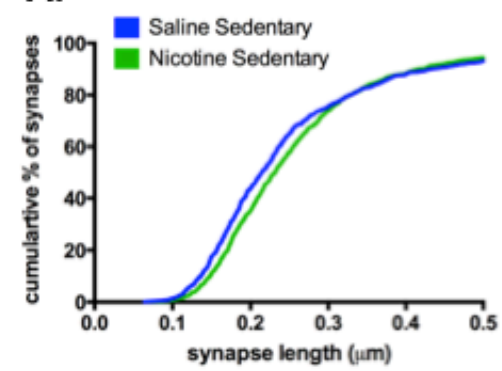

Aii

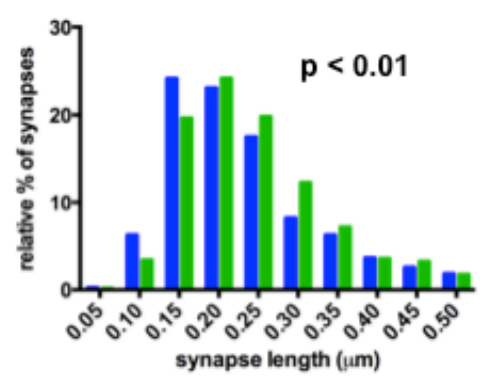

$\mathrm{Bi}$

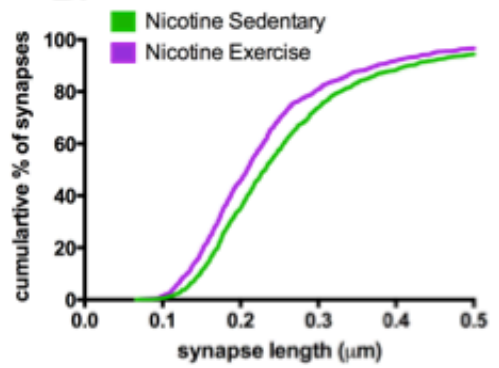

$\mathrm{Bii}$

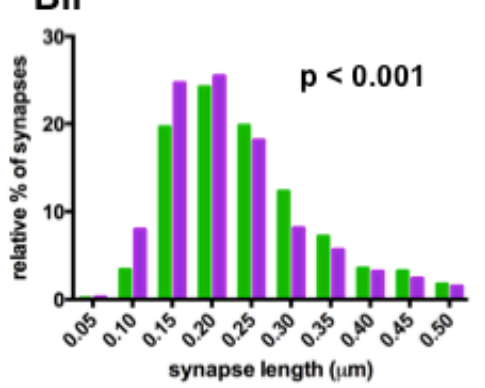

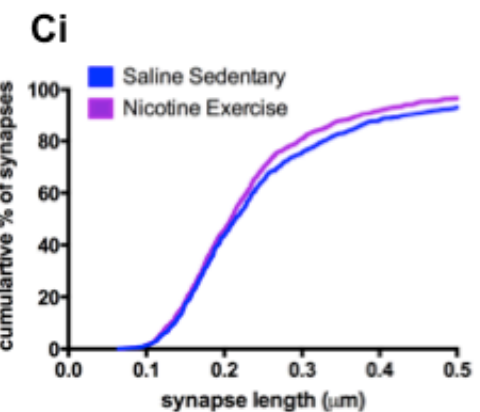

Cii

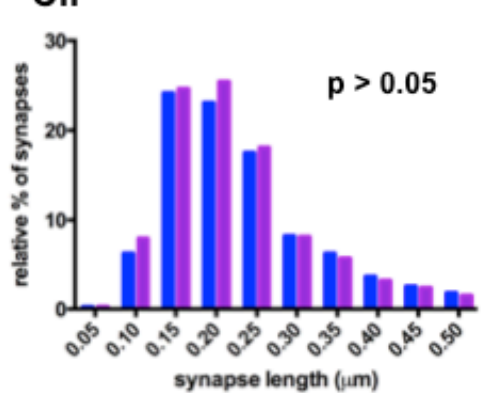

Figure 17 Cumulative (Ai, Bi, and $\mathrm{Ci}$ ) and relative (Aii, Bii, and Cii) freqency plots for asymmetric synapses onto spines in the nucleus accumbens core region. 
The saline exercise group showed a similar synapse length distribution as the saline sedentary however a non-significant trend for larger synapse length was observed $(\mathrm{p}=0.045$; Fig $18 \mathrm{Ai}$ and Aii) and nicotine exercise (Fig $18 \mathrm{Ci}$ and Cii) groups and was also significantly different from the nicotine sedentary group $(\mathrm{p}<0.001$; Fig $18 \mathrm{Bi}$ and Bii). These results indicate that exercise in the saline group did not have a significant effect on synapse length in the nucleus accumbens core.

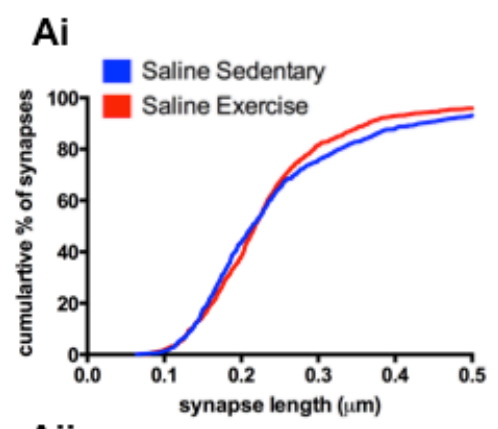

Aii

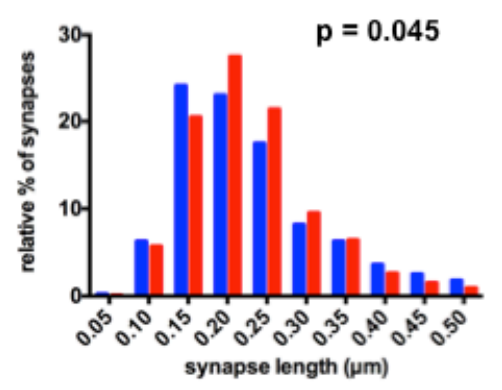

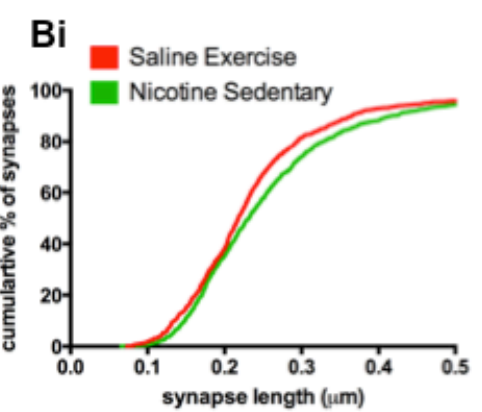

$\mathrm{Bii}$

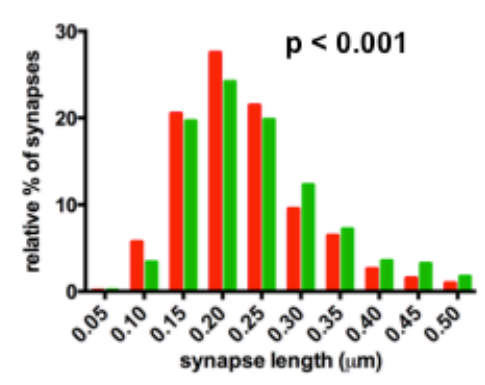

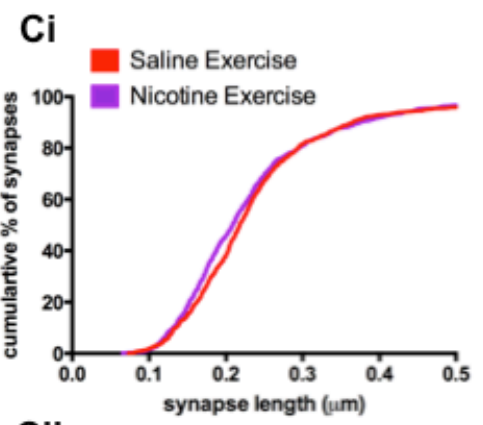

$\mathrm{Cii}$

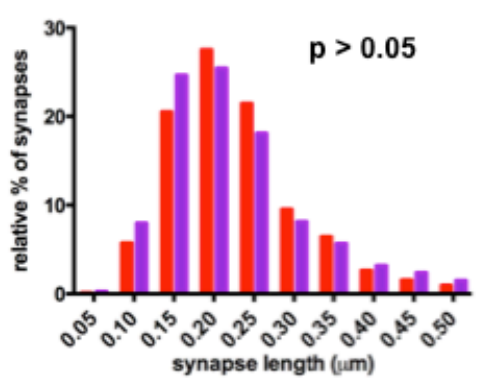

Figure 18 Cumulative (Ai, Bi, and $\mathrm{Ci}$ ) and relative (Aii, Bii, and Cii) freqency plots for asymmetric synapses onto spines in the nucleus accumbens core region. 
Synapse length distribution in the nucleus accumbens shell

The nicotine sedentary group displayed a significant rightward shift in the distribution of synapse length as compared to the saline sedentary group $(\mathrm{p}<0.001$; Fig $19 \mathrm{Ai}$ and Aii). A significant leftward shift in synapse length was observed in the nicotine exercise group as compare to the nicotine sedentary group ( $p<0.001$; Fig $19 \mathrm{Bi}$ and Bii). As seen in the core, the synapse length distribution of the nicotine exercise did not differ from the saline sedentary condition (Fig $19 \mathrm{Ci}$ and Cii). Thus, abstinence from nicotine self-administration led to increased synapse length and exercise during abstinence normalized synapse length back to saline control levels in the shell region.

Ai

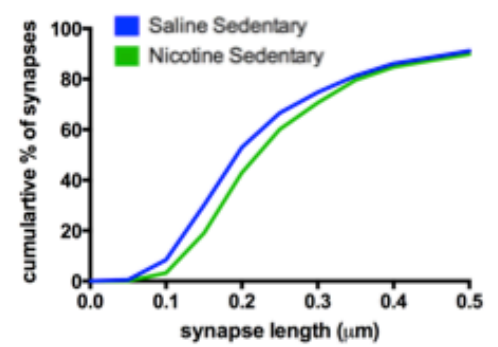

Aii

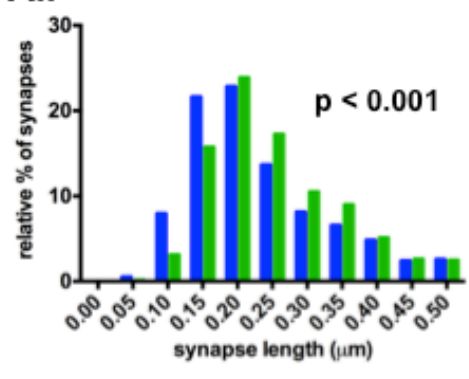

$\mathrm{Bi}$

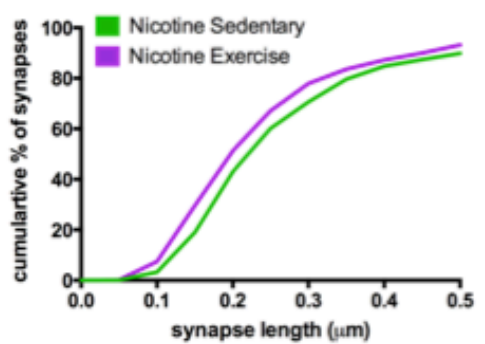

Bii

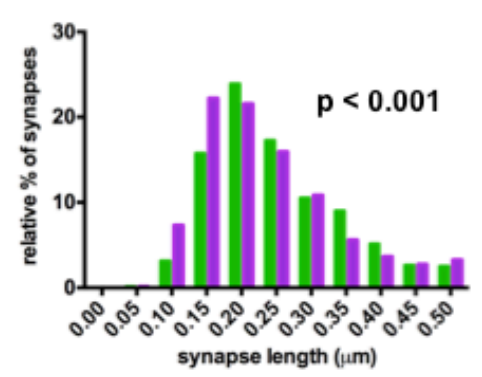

Ci

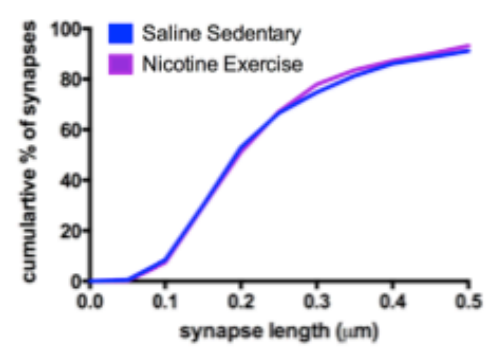

Cii

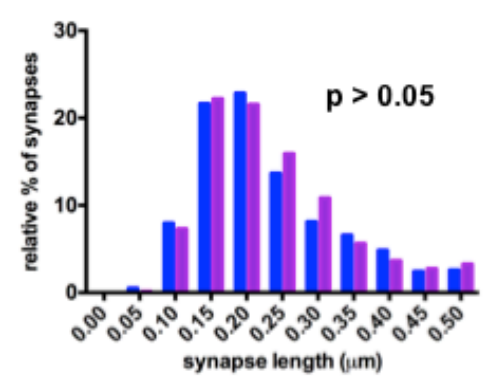

Figure 19 Cumulative (Ai, Bi, and $\mathrm{Ci}$ ) and relative (Aii, Bii, and Cii) freqency plots for asymmetric synapses onto spines in the nucleus accumbens shell region. 
In contrast to findings in the core, a significant rightward shift in the synapse length distribution of the saline exercise was observed as compared to the saline sedentary and nicotine exercise groups (ps $<0.01$; Fig 20 Ai and Aii; Fig $20 \mathrm{Ci}$ and Cii). The saline exercise group synapse length distribution was similar to that of the nicotine sedentary group (Fig $20 \mathrm{Bi}$ and Bii). These results suggest that exercise in the saline control group increased synapse length as compared to the sedentary saline group in a manner similar to that of the nicotine.

Ai

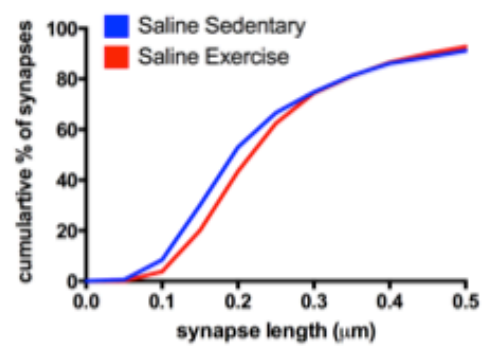

Aii

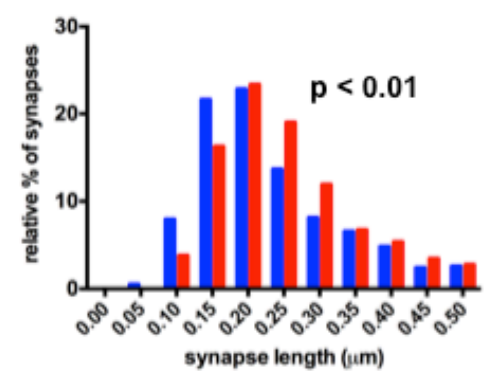

Bi

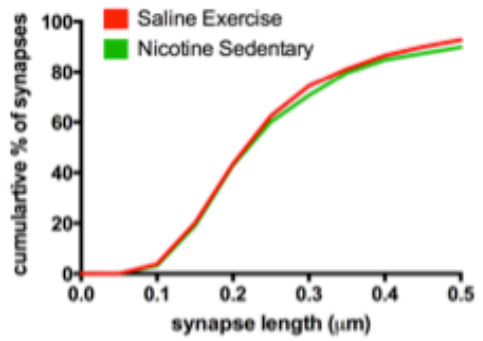

Bii

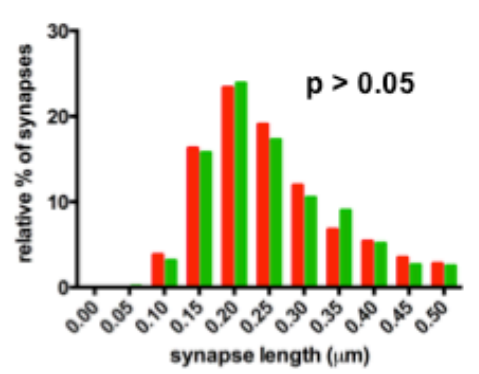

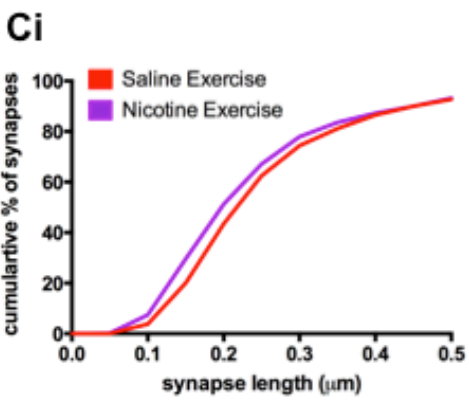

$\mathrm{Cii}$

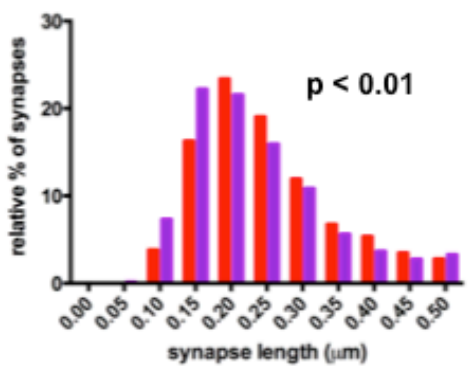

Figure 20 Cumulative (Ai, Bi, and $\mathrm{Ci}$ ) and relative (Aii, Bii, and Cii) freqency plots for asymmetric synapses onto spines in the nucleus accumbens shell region. 


\section{Discussion}

The main finding of the present study is that abstinence from nicotine selfadministration and subsequent nicotine-seeking was associated with an increase in asymmetric synapse length onto spines in the nucleus accumbens core and shell regions. Exercise during abstinence not only significantly attenuated subsequent nicotine-seeking but also normalized synapse length in both regions. These results indicate that the effectiveness of exercise may be associated with its ability to prevent abstinence-induced structural plasticity within the nucleus accumbens.

In this study, we demonstrated that similar to self-administration paradigms in adults (Gipson et al. 2013), there is a long-lasting structural plasticity of dendritic spines within the nucleus accumbens core region of adolescents. Gipson et al. (2013) found an increase in spine head diameter in the nucleus accumbens core following abstinence that was associated with greater nicotine-seeking. The increase in synapse length observed in this study is likely associated with an increase in spine head size as these two measures are directly correlated (i.e., larger spines contain longer synapses; Harris and Stevens 1989). Furthermore, the increased length of the postsynaptic density is likely associated with an increase in AMPA receptor surface expression (Baude et al. 1995; Kharazia and Weinberg 1999; Takumi et al. 1999). Indeed, along with abstinence-induced enlargement of spine head diameter following abstinence, Gipson et al. (2013) observed evidence of increased surface expression of calcium permeable AMPA receptors. In this study we demonstrate that asymmetric synapse length on dendritic spines is also associated with nicotine-seeking, in that groups with higher levels of nicotine-seeking under extinction 
conditions also had longer synapses. Taken together, these results suggest that an increase in larger synapses not only represents an increase in structural plasticity and functional plasticity, but also may underlie the long-term behavioral changes.

Aberrant glutamatergic signaling is believed to be a key component of drugseeking in rodent models (Kalivas and Volkow 2005). An important piece of evidence to support this idea is that the incubation effect of cocaine is associated with a significant increase in the insertion of highly conductive calcium permeable AMPA receptors in the postsynaptic membrane of nucleus accumbens neurons (Conrad et al. 2008; Loweth et al. 2013). Drug-seeking is believed to be mediated by nucleus accumbens core region specifically (Kalivas and Volkow 2005; Everitt and Robbins 2005). However, the nucleus accumbens shell has also been implicated in drug-seeking following abstinence. Specifically, with nicotine there is evidence that glutamate projections to both the core and shell regions of the nucleus accumbens mediates nicotine-seeking (Tessari et al 2004; Liechti et al. 2007; Bespalov et al. 2008; Palmatier et al. 2007; Gipson et al. 2013), and in studies with cocaine, activation of the ventral medial prefrontal cortex, which sends projections to the shell region (Voorn et al. 2004), has been shown to be important for cue-induced reinstatement. Furthermore, changes in calcium permeable AMPA receptor surface expression associated with the incubation of craving for cocaine has also been observed in the shell region following prolonged abstinence but to a lesser extent than in core (Conrad et al. 2008). In the present study we found increased glutamatergic synaptic plasticity in both the core and shell regions. Since exercise attenuated this effect in both regions it is unclear which region is associated with nicotine-seeking in adolescents. 
The most interesting finding in the current study was that exercise appeared to normalize abstinence-induced changes of synapse length within the nucleus accumbens. This effect may be due to the ability of exercise to mimic nicotine's actions within the brain and thus prevent abstinence-induced plasticity. Although, glutamatergic signaling induced by exercise within the nucleus accumbens shell region specifically has not yet been examined, some evidence indicates that exercise can modulate glutamate and dopamine neurotransmission within the striatum. Specifically, a microdialysis study has demonstrated that during exercise, extracellular glutamate and dopamine levels are increased in the striatum (Meeusen et al. 1997). Given that exercise has been shown to be rewarding (Lett et al. 2000, 2001; Greenwood et al. 2011; Belke 1997; 2000; Belke and Wagner 2005; Brene et al. 2007; Iversen 1993), it is possible that exercise could also affect glutamate and dopamine release within the nucleus accumbens as well. One caveat to these findings is that exercise induced an increase in synapse length in the nucleus accumbens shell of saline control rats in a manner that was similar to the effect of abstinence from nicotine. There is also some precedence within other brain regions to indicate that running can affect structural plasticity. Within the hippocampus, exercise has previously been shown to increase dendrite length and complexity as well as increase spine density (Stranahan et al. 2007; Eadie at al. 2005; Redila and Christie 2006). The functional significance of this increased synapse length 1-day after the last exercise session within the shell is not clear. Interestingly, prolonged access to wheel running followed by 25 days without running did not have an affect of spine density in the nucleus accumbens shell (Robinson and Kolb 1999), suggesting that the increase in 
synapse length observed in the present study may not be a persistent alteration in plasticity.

In summary, the effectiveness of exercise in reducing nicotine-seeking may involve its ability to prevent abstinence-induced plasticity of glutamatergic connectivity within the nucleus accumbens. Further work is necessary to determine if this effect of exercise is due to an alteration of glutamatergic projections to the core or shell regions or both. Future studies should also aim to elucidate the circuitry involved in both abstinence-induced plasticity and the efficacy of exercise. 


\section{Chapter 6 General Discussion}

The work presented in this dissertation was designed to examine the efficacy of exercise as a prevention and intervention in an adolescent-onset model of nicotine addiction. In addition, this work was the first attempt to identify structural plasticity associated with nicotine-seeking and its modulation by exercise. In the following sections, I will summarize findings from my dissertation and discuss their potential implications. Future directions are also suggested.

\section{Behavioral effects of exercise}

In Chapter 2, the behavioral effect of exercise on the initiation of nicotine selfadministration was examined. This study demonstrated that specifically exercise, and not the environmental enrichment provided by the presence of a wheel, robustly attenuated vulnerability to initiate nicotine self-administration in adolescent rats. This finding supports a growing body of literature suggesting that exercise can prevent the initiation of drug use (Smith and Pitts 2011; Engelmann et al. 2013), and extends this previous work to nicotine self-administration and to adolescence, a developmental stage known to be critical in drug use initiation. In light of epidemiological findings of lower smoking rates in active adolescents as compared to their less active peers (e.g., Pastor et al. 2003; Escobedo et al. 1993; Field et al. 2001; Melnick et al. 2001; Naylor et al. 2001; Pate et al. 1996, 2000; Rainey et al. 1996; Mattila et al. 2012; Castrucci et al. 2004), this work suggests that the effects of exercise are not solely due to social influences and norms but 
are also biologically based. The implications of our findings are important and suggest that future tobacco prevention programs should include an exercise component.

In Chapters 3, 4, and 5 of this dissertation, the behavioral effect of exercise on the relapse vulnerability was examined. Results from these experiments consistently demonstrated that exercise during an abstinence period attenuated subsequent nicotineseeking under extinction conditions. These studies also demonstrated that the use of an adolescent-onset nicotine self-administration paradigm leads to robust nicotine-seeking in both males and females. Similar to findings in Chapter 2, in males, the protective effect of wheel-running was due to exercise, and not the environmental enrichment provided by the wheel itself. In contrast, females displayed a reduced sensitivity to nicotine-seeking under both the unlocked exercise condition and the locked wheel control condition. There are several interpretations of these results. First, females may use the locked wheel condition in a manner that provides a non-aerobic form of exercise and are thus more sensitive to the effects of exercise than males. Second, females may be more sensitive than males to the environmental enrichment provided by a wheel and this effect is similar to the effectiveness of exercise in females. Third, exercise may not account for the attenuated nicotine-seeking in females and this effect may be due to environmental enrichment alone. However, given evidence showing that females are more active (Hitchcock 1925; Eikelboom and Mills 1988; Smith et al. 2011; 2012) and given results with other drugs of abuse indicating that wheel running can reduce cocaine selfadministration as well as cocaine-seeking following abstinence in females (Cosgrove et al. 2002; Zlebnik et al. 2010; 2012), the most parsimonious explanation is that adolescent 
females are more sensitive to alternative forms of exercise within locked running wheel. Future preclinical research should directly compare the effects of nonaerobic models of exercise between adolescent males and females to examine this possibility. Future clinical research may consider including various types of exercise, aerobic and nonaerobic forms, as part of cessation treatment in adolescent boys and girls.

\section{Neurobiological effects of exercise}

In Chapter 5, structural plasticity within the nucleus accumbens following abstinence from adolescent-onset nicotine self-administration was analyzed with and without exercise. Nicotine self-administration followed by abstinence resulted in an enlargement of synapse length and exercise normalized these levels in both the nucleus accumbens core and shell regions. These results were observed in asymmetric synapses onto spines, which are known to be excitatory synapses (Nimchinsky et al. 2002; Gray 1959), suggesting that the behavioral attenuation of nicotine-seeking may be due to structural attenuation of glutamatergic plasticity.

An interesting finding in the experiment described in Chapter 5 was the observed increase in plasticity within both regions of the nucleus accumbens. These findings can be reconciled in a couple of ways. First, enhanced glutamatergic plasticity in both regions may be important for the drug-seeking behavior. Second, one or both of the regions may express an increase in plasticity that is not related to drug-seeking behavior. In support of the latter, Li et al. (2004) found that cocaine-induced behavioral sensitization following an abstinence period from experimenter administered cocaine was associated with 
increased spine density in the core region specifically. An increase in spine density occurred in the absence of behavioral sensitization within the nucleus accumbens shell region suggesting that structural plasticity within the shell is a consequence of the history with the drug and not related to the behavior. However, although it is popularly believed that medial prefrontal cortex projections to the nucleus accumbens core region underlie drug-seeking (Everitt and Robbins 2005; Fischer-Smith et al. 2012; Hotsenpiller et al. 2001; Kalivas and Volkow 2005; McFarland et al. 2003; Gipson et al. 2013), there is evidence that projections to the shell region are involved as well (Koya et al. 2009; Bossert et al. 2013). The result from this study support the idea that at least the glutamatergic plasticity associated with late abstinence is present in both the core and shell regions of the nucleus accumbens.

The neurobiological mechanism of exercise that led to a behavioral attenuation of relapse to vulnerability and the observed normalization of synapse length are likely a result of its effects on the mesolimbic reward pathway. As discussed in the Chapter 1, extended access drug self-administration and early withdrawal are associated with lower glutamate and dopamine receptor function in the nucleus accumbens (Ahmed and Koob 2004; Koeltzow and White 2003; Hao et al. 2010). The persistent absence of drug during abstinence leads to neuroadaptations that that render neurons within the nucleus accumbens more sensitive to dopamine and glutamate (Schmidt and Pierce 2010). In animal models, exercise has been shown to increase glutamate and dopamine overflow in the striatum (Meeusen et al. 1997). Thus, the addition of exercise may maintain dopamine and glutamate levels and thus prevent the induction of plasticity associated with the 
absence of drug. Therefore, exercise during abstinence likely prevented the increase in glutamatergic plasticity observed in Chapter 5 rather than reduced nicotine induced glutamatergic plasticity.

Although not examined in this dissertation, I would like to speculate on the neurobiological mechanism underlying behavioral attenuation of acquisition to nicotine self-administration. In animal models, exercise has been found to be rewarding with animals developing a conditioned place preference for environments paired with the after effects of exercise (Lett et al. 2000, 2001; Greenwood et al. 2011) and performing an operant response to gain access to a running wheel (Belke 1997; 2000; Belke and Wagner 2005; Brene et al. 2007; Iversen 1993). Similarly, as discussed above exercise increases dopamine release in the striatum with increased levels observed for at least several hours following exercise (Meeusen et al. 1997). It is possible that exercise prevented a further elevation in nucleus accumbens dopamine levels induced by nicotine self-administration and thus blunted the reinforcing effects of nicotine. Indeed, it has previously been shown that exercise blunts MDMA-induced increases in dopamine overflow in the nucleus accumbens along with MDMA-induced conditioned place preference (Chen et al. 2008). Further work is necessary to determine if and how exercise might affect dopamine signaling following nicotine self-administration in adolescent rats.

One caveat to the prevention and plasticity studies of this dissertation is that they were conducted in males only. As discussed in Chapters 1 and 4 of this dissertation, males and females differ on many different aspects of nicotine addiction including vulnerability to acquire nicotine self-administration (Lynch et al. 2002; Donny et al. 
2000; Lynch 2009; Torres et al. 2008; Torres et al. 2009). Given that females differed from males in their sensitivity to the effects of exercise in preventing nicotine-seeking, I would hypothesize that similar sex-differences would be observed in preventing acquisition of nicotine self-administration. Specifically, females would show slowed and reduced rates under exercise unlocked wheel and control locked wheel conditions as compared to females that did not have access to a running wheel prior to acquisition of nicotine self-administration testing. Incredibly, to date there are no published studies on sex differences in drug abstinence-induced structural plasticity. Given that females have been shown to have a greater number of dendritic spines and larger spines within the nucleus accumbens as compared to males (Forlano and Woolley 2009; Wissman et al. 2012), it is possible that structural plasticity induced by abstinence may also be sexually dimorphic.

In summary, voluntary exercise prior to drug self-administration during adolescence reduces acquisition vulnerability suggesting that exercise per se may impart protection for smoking initiation in human adolescents. Exercise during abstinence also attenuated nicotine-seeking in an adolescent-onset model of nicotine addiction, suggesting that exercise may help adolescent smokers maintain smoking abstinence by reducing craving. However, since the efficacy of exercise in attenuating nicotine-seeking was not dissociable from the environmental enrichment effect in females, further preclinical and clinic work is needed to elucidate sex-differences in sensitivity to exercise as a prevention and intervention for nicotine addiction. Finally, exercise during abstinence was found to normalize nicotine-induced structural plasticity of excitatory 
synapses in the nucleus accumbens, suggesting that the mechanism underlying the efficacy of exercise may be its ability to modulate abstinence-induced plasticity. Together, the results from this dissertation demonstrate that exercise, by modulating plasticity within the reward pathway, may be an effective prevention and intervention for adolescent-onset nicotine addiction. 


\section{References}

Aaron DJ, Dearwater SR, Anderson R, Olsen T, Kriska AM, Laporte RE (1995) Physical activity and the initiation of high-risk health behaviors in adolescents. Med Sci Sports Exerc 27:1639-1645.

Abdolahi A, Acosta G, Breslin FJ, Hemby SE, Lynch WJ (2010) Incubation of nicotineseeking is associated with enhanced protein kinase A-regulated signaling of dopamine- and cAMP-regulated phosphoprotein of $32 \mathrm{kDa}$ in the insular cortex. Eur J Neurosci 31

Adriani W, Macrì S, Pacifici R, Laviola G (2002) Peculiar vulnerability to nicotine oral self-administration in mice during early adolescence. Neuropsychopharmacology $27: 212-224$.

Ahmed SH, Koob GF (2004) Changes in response to a dopamine receptor antagonist in rats with escalating cocaine intake. Psychopharmacology (Berl ) 172:450-454.

Ahmed SH, Lin D, Koob GF, Parsons LH (2003) Escalation of cocaine selfadministration does not depend on altered cocaine-induced nucleus accumbens dopamine levels. J Neurochem 86:102-113.

Ahmed S, Koob G (1998) Transition from moderate to excessive drug intake: change in hedonic set point. Science 282:298-300. 
Anker JJ, Carroll ME (2010) Reinstatement of cocaine seeking induced by drugs, cues, and stress in adolescent and adult rats. Psychopharmacology 208: 211-222.

Baude A, Nusser Z, Molnar E, McIlhinney R, Somogyi P (1995) High-resolution immunogold localization of AMPA type glutamate receptor subunits at synaptic and non-synaptic sites in rat hippocampus. Neuroscience 69:1031-1055.

Bedi G, Preston KL, Epstein DH, Heishman SJ, Marrone GF, Shaham Y, de Wit H (2011) Incubation of cue-induced cigarette craving during abstinence in human smokers. Biol Psychiatry 69: 708-171.

Belke TW (1997) Running and responding reinforced by the opportunity to run: effect of reinforcer duration. J Exp Anal Behav 67: 337-351.

Belke TW (2000) Studies of wheel-running reinforcement: parameters of Herrnstein's (1970) response-strength equation vary with schedule order. J Exp Anal Behav 73: $319-331$.

Belke TW, Wagner JP (2005) The reinforcing property and the rewarding aftereffect of wheel running in rats: a combination of two paradigms. Behav Processes 68:165172.

Belluzzi JD, Lee AG, Oliff HS, Leslie FM (2004) Age-dependent effects of nicotine on locomotor activity and conditioned place preference in rats. Psychopharmacology (Berl ) 174:389-395.

Ben-Shahar OM, Szumlinski KK, Lominac KD, Cohen A, Gordon E, Ploense KL, DeMartini J, Bernstein N, Rudy NM, Nabhan AN (2012) Extended access to 
cocaine self-administration results in reduced glutamate function within the medial prefrontal cortex. Addict Biol 17:746-757.

Bergstrom HC, McDonald CG, French HT, Smith RF (2008) Continuous nicotine administration produces selective, age-dependent structural alteration of pyramidal neurons from prelimbic cortex. Synapse 62:31-39.

Bespalov AY, Dravolina OA, Sukhanov I, Zakharova E, Blokhina E, Zvartau E, Danysz W, van Heeke G, Markou A (2005) Metabotropic glutamate receptor (mGluR5) antagonist MPEP attenuated cue-and schedule-induced reinstatement of nicotine self-administration behavior in rats. Neuropharmacology 49:167-178.

Bock BC, Marcus BH, King TK, Borrelli B, Roberts MR (1999) Exercise effects on withdrawal and mood among women attempting smoking cessation. Addict Behav 24: 399-410.

Boles SM, Johnson PB (2001) Gender, weight concerns, and adolescent smoking. $J$ Addict Dis 20: 5-14.

Bossert JM, Stern AL, Theberge FR, Marchant NJ, Wang H, Morales M, Shaham Y (2012) Role of projections from ventral medial prefrontal cortex to nucleus accumbens shell in context-induced reinstatement of heroin seeking. The Journal of Neuroscience 32:4982-4991.

Brene S, Bjornebekk A, Aberg E, Mathe AA, Olson L, Werme M (2007) Running is rewarding and antidepressive. Physiol Behav 92: 136-140. 
Breslau N, Fenn N, Peterson EL (1993) Early smoking initiation and nicotine dependence in a cohort of young adults. Drug Alcohol Depend 33:129-137.

Breslau N, Peterson EL (1996) Smoking cessation in young adults: Age at initiation of cigarette smoking and other suspected influences. Am J Public Health 86: 214220.

Brielmaier JM, McDonald CG, Smith RF (2007) Immediate and long-term behavioral effects of a single nicotine injection in adolescent and adult rats. Neurotoxicol Teratol 29:74-80.

Brown RW, Kolb B (2001) Nicotine sensitization increases dendritic length and spine density in the nucleus accumbens and cingulate cortex. Brain Res 899:94-100.

Cadoni C, Di Chiara G (2000) Differential changes in accumbens shell and core dopamine in behavioral sensitization to nicotine. Eur J Pharmacol 387:R23-R25.

Cadoni C, Di Chiara G (2000) Differential changes in accumbens shell and core dopamine in behavioral sensitization to nicotine. Eur J Pharmacol 387:R23-R25.

Caine SB, Koob GF (1994) Effects of mesolimbic dopamine depletion on responding maintained by cocaine and food. J Exp Anal Behav 61: 213-221.

Caggiula AR, Donny EC, Palmatier MI, Liu X, Chaudhri N, Sved AF (2009) The role of nicotine in smoking: a dual-reinforcement model. In: Anonymous The Motivational Impact of Nicotine and its Role in Tobacco Use. Springer, pp 91109. 
Caggiula AR, Donny EC, White AR, Chaudhri N, Booth S, Gharib MA, Hoffman A, Perkins KA, Sved, AF (2001) Cue dependency of nicotine self-administration and smoking. Pharmacol Biochem Behav 70: 515-530.

Calabresi P, Lacey M, North R (1989) Nicotinic excitation of rat ventral tegmental neurones in vitro studied by intracellular recording. Br J Pharmacol 98:135-140.

Campbell UC, Carroll ME (2000) Acquisition of drug self-administration: Environmental and pharmacological interventions. Exp Clin Psychopharmacol 8:312.

Carboni E, Imperato A, Perezzani L, Di Chiara G (1989) Amphetamine, cocaine, phencyclidine and nomifensine increase extracellular dopamine concentrations preferentially in the nucleus accumbens of freely moving rats. Neuroscience 28:653-661.

Castrucci BC, Gerlach KK, Kaufman NJ, Orleans CT (2004) Tobacco use and cessation behavior among adolescents participating in organized sports. Am J Health Behav 28:63-71.

Cavallo DA, Smith AE, Schepis TS, Desai R, Potenza MN, Krishnan-Sarin S (2010) Smoking expectancies, weight concerns, and dietary behaviors in adolescence. Pediatrics 126: e66-72. 
Cepeda-Benito A, Reynoso JT, Erath S (2004) Meta-analysis of the efficacy of nicotine replacement therapy for smoking cessation: differences between men and women. J Consult Clin Psychol 72:712.

Chaudhri N, Caggiula AR, Donny EC, Booth S, Gharib MA, Craven LA, Allen SS, Sved AF, Perkins KA (2005) Sex differences in the contribution of nicotine and nonpharmacological stimuli to nicotine self-administration in rats. Psychopharmacology (Berl ) 180:258-266.

Chauvet C, Lardeux V, Goldberg SR, Jaber M, Solina M (2009) Environmental enrichment reduces cocaine seeking and reinstatement induced by cues and stress but not by cocaine. Neuropsychopharmacology 34: 2767-2778.

Chen H, Matta SG, Sharp BM (2007) Acquisition of nicotine self-administration in adolescent rats given prolonged access to the drug, Neuropsychopharmacology 32: 700-709.

Chen HI, Kuo YM, Liao C, Jen CJ, Huang AM, Cherng CG, Su S, Yu L (2008) Longterm compulsive exercise reduces the rewarding efficacy of 3, 4methylenedioxymethamphetamine. Behav Brain Res 187:185-189.

Chen J, Millar WJ (1998) Age of smoking initiation: implications for quitting. HEALTH REPORTS-STATISTICS CANADA 9:39-48.

Colby SM, Tiffany ST, Shiffman S, Niaura RS (2000) Are adolescent smokers dependent on nicotine? A review of the evidence. Drug Alcohol Depend 59:83-95. 
Collingwood TR, Sunderlin J, Reynolds R, Kohl HW (2000) Physical training as a substance abuse prevention intervention for youth. J Drug Educ 30:435-452.

Conrad KL, Tseng KY, Uejima JL, Reimers JM, Heng L, Shaham Y, Marinelli M, Wolf ME (2008) Formation of accumbens GluR2-lacking AMPA receptors mediates incubation of cocaine craving. Nature 454:118-121.

Corrigall WA, Coen KM, Adamson KL (1994) Self-administered nicotine activates the mesolimbic dopamine system through the ventral tegmental area. Brain Res $653: 278-284$

Corrigall WA, Franklin KB, Coen KM, Clarke PB (1992) The mesolimbic dopaminergic system is implicated in the reinforcing effects of nicotine. Psychopharmacology (Berl) 107: 285-289.

Cosgrove KP, Hunter RG, Carroll ME (2002) Wheel-running attenuates intravenous cocaine self-administration in rats: sex differences. Pharmacol Biochem Behav. 73: $663-71$.

Counotte DS, Smit AB, Pattij T, Spijker S (2011) Development of the motivational system during adolescence, and its sensitivity to disruption by nicotine. Dev Cogn Neurosci 1: 430-443.

Crombag HS, Gorny G, Li Y, Kolb B, Robinson TE (2005) Opposite effects of amphetamine self-administration experience on dendritic spines in the medial and orbital prefrontal cortex. Cerebral Cortex 15:341-348. 
Dalley JW, Fryer TD, Brichard L, Robinson ES, Theobald DE, Lääne K, Pena Y, Murphy ER, Shah Y, Probst K, Abakumova I, Aigbirhio FI, Richards HK, Hong Y, Baron JC, Everitt BJ, Robbins TW (2007) Nucleus accumbens D2/3 receptors predict trait impulsivity and cocaine reinforcement. Science 315: 1267-1270.

Danaei G, Ding EL, Mozaffarian D, Taylor B, Rehm J, et al. (2009) The Preventable Causes of Death in the United States: Comparative Risk Assessment of Dietary, Lifestyle, and Metabolic Risk Factors. PLoS Med 6(4): e1000058. doi:10.1371/journal.pmed.1000058

Di Chiara G, Imperato A (1988) Drugs abused by humans preferentially increase synaptic dopamine concentrations in the mesolimbic system of freely moving rats. Proceedings of the National Academy of Sciences 85:5274-5278.

Diaz Heijtz R, Kolb B, Forssberg H (2003) Can a therapeutic dose of amphetamine during pre-adolescence modify the pattern of synaptic organization in the brain? Eur J Neurosci 18:3394-3399.

Diergaarde L, de Vries W, Raaso H, Schoffelmeer ANM, De Vries TJ (2008) Contextual renewal of nicotine-seeking in rats and its suppression by the cannabinoid-1 receptor antagonist Rimonabant (SR141716A). Neuropharmacology 55: 712-716.

DiFranza JR, Savageau JA, Rigotti NA, Fletcher K, Ockene JK, McNeill AD, Coleman M, Wood C (2002) Development of symptoms of tobacco dependence in youths: 30 month follow up data from the DANDY study. Tob Control 11:228-235. 
Donny EC, Caggiula AR, Rowell PP, Gharib MA, Maldovan V, Booth S, Mielke MM, Hoffman A, McCallum S (2000) Nicotine self-administration in rats: estrous cycle sex differences and nicotinic receptor binding. Psychopharmacology 151: $392-405$.

Døssing M (1985) Effect of acute and chronic exercise on hepatic drug metabolism. Clin Pharmacokinet 10: 426-431.

Dravolina OA, Zakharova ES, Shekunova EV, Zvartau EE, Danysz W, Bespalov AY (2007) mGlu1 receptor blockade attenuates cue-and nicotine-induced reinstatement of extinguished nicotine self-administration behavior in rats. Neuropharmacology 52:263-269.

Eadie BD, Redila VA, Christie BR (2005) Voluntary exercise alters the cytoarchitecture of the adult dentate gyrus by increasing cellular proliferation, dendritic complexity, and spine density. J Comp Neurol 486:39-47.

Eikelboom R, Mills R (1988) A microanalysis of wheel running in male and female rats. Physiol Behav 43: 625-630.

Ekkekakis P, Hall EE, Petruzzello SJ (2008) The relationship between exercise intensity and affective responses demystified: To crack the 40-year-old nut, repace the 40year-old nutcracker. Ann Behav Med 35: 136-149.

El Rawas R, Thiriet N, Lardeux V, Jaber M, Solinas M (2009) Environmental enrichment decreases the rewarding but not the activating effects of heroin.

Psychopharmacology (Berl ) 203:561-570. 
Engelmann AJ, Aparicio MB, Kim A, Sobieraj JC, Yuan CJ, Grant Y, Mandyam CD (2013) Chronic wheel running reduces maladaptive patterns of methamphetamine intake: regulation by attenuation of methamphetamine-induced neuronal nitric oxide synthase. Brain Structure and Function:1-16.

Escobedo LG, Marcus SE, Holtzman D, Giovino GA (1993) Sports participation, age at smoking initiation, and the risk of smoking among US high school students.

JAMA: the journal of the American Medical Association 269:1391-1395.

Everitt BJ, Robbins TW (2005) Neural systems of reinforcement for drug addiction: from actions to habits to compulsion. Nat Neurosci 8:1481-1489.

Feltenstein MW, Ghee SM, See RE (2012) Nicotine self-administration and reinstatement of nicotine-seeking in male and female rats. Drug Alcohol Depend 121: 240-246.

Ferrario CR, Gorny G, Crombag HS, Li Y, Kolb B, Robinson TE (2005) Neural and behavioral plasticity associated with the transition from controlled to escalated cocaine use. Biol Psychiatry 58:751-759.

Field T, Diego M, Sanders CE (2001) Exercise is positively related to adolescents' relationships and academics. Adolescence.

Fischer-Smith KD, Houston AC, Rebec GV (2012) Differential effects of cocaine access and withdrawal on glutamate type 1 transporter expression in rat nucleus accumbens core and shell. Neuroscience 210:333-339. 
Fontes-Ribeiro C, Marques E, Pereira F, Silva A, Macedo TA (2011) May exercise prevent addiction? Current neuropharmacology 9:45.

Forlano PM, Woolley CS (2010) Quantitative analysis of pre- and postsynaptic sex differences in the nucleus accumbens. J Comp Neurol 518: 1330-1348.

Giedd JN (2004) Structural magnetic resonance imaging of the adolescent brain. Ann. N.Y. Acad. Sci. 1021: 77-85.

Gipson CD, Reissner KJ, Kupchik YM, Smith AC, Stankeviciute N, Hensley-Simon ME, Kalivas PW (2013) Reinstatement of nicotine seeking is mediated by glutamatergic plasticity. Proceedings of the National Academy of Sciences 110:9124-9129.

Gonzalez CL, Gharbawie OA, Whishaw IQ, Kolb B (2005) Nicotine stimulates dendritic arborization in motor cortex and improves concurrent motor skill but impairs subsequent motor learning. Synapse 55:183-191.

Gray EG (1959) Axo-somatic and axo- dendritic synapses of the cerebral cor- tex: an electron microscope study. J. Anat. 93: 420-433.

Greenwood BN, Foley TE, Le TV, Strong PV, Loughridge AB, Day HE, Fleshner M (2011) Long-term voluntary wheel running is rewarding and produces plasticity in the mesolimbic reward pathway. Behav Brain Res 217:354-362.

Grimm JW, Hope BT, Wise RA, Shaham Y (2001) Incubation of cocaine craving after withdrawal. Nature 412: 1410142. 
Guezennec C, Abdelmalki A, Seirurier B, Merino D, Bigard X, Berthelot M, Pierard C, Peres M (1998) Effects of prolonged exercise on brain ammonia and amino acids. Int J Sports Med 19:323-327.

Kaplan G, Ivanov I (2011) Pharmacotherapy for substance abuse disorders in adolescence. Pediatr Clin North Am 58: 243-258.

Haasova M, Warren FC, Ussher M, Janse Van Rensburg K, Faulkner G, Cropley M, Byron-Daniel J, Everson-Hock ES, Oh H, Taylor AH (2013) The acute effects of physical activity on cigarette cravings: systematic review and meta-analysis with individual participant data. Addiction 108: 26-37.

Hamilton DA, Kolb, B (2005) Differential effects of nicotine and complex housing on subsequent experience-dependent structural plasticity in the nucleus accumbens. Behav Neurosci 119: 355-365.

Hao Y, Martin-Fardon R, Weiss F (2010) Behavioral and functional evidence of metabotropic glutamate receptor $2 / 3$ and metabotropic glutamate receptor 5 dysregulation in cocaine-escalated rats: factor in the transition to dependence. Biol Psychiatry 68:240-248.

Harris KM, Stevens JK (1989) Dendritic spines of CA 1 pyramidal cells in the rat hippocampus: serial electron microscopy with reference to their biophysical characteristics. The Journal of neuroscience 9:2982-2997.

Hemby SE, Koves TR, Smith JE, Dworkin SI (1997) Differences in extracellular dopamine concentrations in the nucleus accumbens during response-dependent 
and response-independent cocaine administration in the rat. Psychopharmacology (Berl ) 133:7-16.

Hitchcock F (1925) STUDIES IN VIGOR V. The Comparative Activity of Male and Female Albino Rats. American Journal of Physiology--Legacy Content 75:205210.

Horn K, Dino G, Branstetter SA, Zhang J, Noerachmanto N, Jarrett T, Taylor M (2011) Effects of physical activity on teen smoking cessation. Pediatrics 128: 801-811. Horn K, Branstetter S, Zhang J, Jarrett T, Tompkins NO, Anesetti-Rothermel A, Olfert M, Richards T, Dino G (2013) Understanding physical activity outcomes as a function of teen smoking cessation. $J$ Adolesc Health doi: 10.1016/j.jadohealth.2013.01.019. [Epub ahead of print]

Hosseini M, Alaei HA, Naderi A, Sharifi MR, Zahed R (2009) Treadmill exercise reduces self-administration of morphine in male rats. Pathophysiology 16:3-7.

Hotsenpiller G, Giorgetti M, Wolf ME (2001) Alterations in behaviour and glutamate transmission following presentation of stimuli previously associated with cocaine exposure. Eur J Neurosci 14:1843-1855.

Hu M, Davies M, Kandel DB (2006) Epidemiology and correlates of daily smoking and nicotine dependence among young adults in the United States. Am J Public Health 96:299-308. 
Huxley RR, Woodward M (2011) Cigarette smoking as a risk factor for coronary heart disease in women compared with men: a systematic review and meta-analysis of prospective cohort studies. The Lancet 378:1297-1305.

Imperato A, Di Chiara G (1986) Preferential stimulation of dopamine release in the nucleus accumbens of freely moving rats by ethanol. J Pharmacol Exp Ther 239:219-228.

Imperato A, Mulas A, Di Chiara G (1986) Nicotine preferentially stimulates dopamine release in the limbic system of freely moving rats. Eur J Pharmacol 132:337-338.

Iversen IH (1993) Techniques for establishing schedules with wheel running as reinforcement in rats. $J$ Exp Anal Behav 60: 219-238.

Janse Van Rensburg K, Taylor A, Hodgson T (2009) The effects of acute exercise on attentional bias towards smoking related stimuli during temporary abstinence from smoking. Addiction 104:1910-1917.

Japuntich SJ, Leventhal AM, Piper ME, Bolt DM, Roberts LJ, Fiore MC, Baker TB (2011) Smoker characteristics and smoking-cessation milestones. Am J Prev Med 40:286-294.

Kalivas PW, Volkow ND (2005) The neural basis of addiction: a pathology of motivation and choice. Am J Psychiatry 162:1403-1413.

Kalivas PW, McFarland K (2003) Brain circuitry and the reinstatement of cocaineseeking behavior. Psychopharmacology (Berl ) 168:44-56. 
Kaplan G, Ivanov I (2011) Pharmacotherapy for substance abuse disorders in adolescence. Pediatr Clin North Am 58: 243-258.

Kerstetter KA, Aguilar VR, Parrish AB, Kippin TE (2008) Protracted time-dependent increases in cocaine-seeking behavior during cocaine withdrawal in female rats relative to male rats. Pyscohpharmacology 198: 63-75.

Kharazia V, Weinberg R (1999) Immunogold localization of AMPA and NMDA receptors in somatic sensory cortex of albino rat. J Comp Neurol 412:292-302.

Khuder SA, Dayal HH, Mutgi AB (1999) Age at smoking onset and its effect on smoking cessation. Addict Behav 24:673-677.

Kirkcaldy BD, Shephard RJ, Siefen RG (2002) The relationship between physical activity and self-image and problem behaviour among adolescents. Soc Psychiatry Psychiatr Epidemiol 37:544-550.

Klein LC, Stine MM, Vandenbergh DJ, Whetzel CA, Kamens HM (2004) Sex differences in voluntary oral nicotine consumption by adolescent mice: a doseresponse experiment. Pharmacology Biochemistry and Behavior 78:13-25.

Klein LC, Stine MM, Vandenbergh DJ, Whetzel CA, Kamens HM (2004) Sex differences in voluntary oral nicotine consumption by adolescent mice: a doseresponse experiment. Pharmacology Biochemistry and Behavior 78:13-25. 
Klesges RC, Elliott VE, Robinson LA (1997) Chronic dieting and the belief that smoking controls body weight in a biracial population-based adolescent sample. Tob Conttrol 6: 89-94.

Koeltzow TE, White FJ (2003) Behavioral depression during cocaine withdrawal is associated with decreased spontaneous activity of ventral tegmental area dopamine neurons. Behav Neurosci 117:860.

Kolb B, Gorny G, Söderpalm AH, Robinson TE (2003) Environmental complexity has different effects on the structure of neurons in the prefrontal cortex versus the parietal cortex or nucleus accumbens. Synapse 48:149-153.

Korhonen T, Kujala UM, Rose RJ, Kaprio J (2009) Physical activity in adolescence as a predictor of alcohol and illicit drug use in early adulthood: a longitudinal population based twin study. Twin research and human genetics: the official journal of the International Society for Twin Studies 12:261.

Koteja P, Garland T Jr, Sax JK, Swallow JG, and Carter PA (1999) Behaviour of house mice artificially selected for high levels of voluntary wheel running. Anim Behav 58: $1307-1318$.

Kota D, Martin BR, Robinson SE, Damaj MI (2007) Nicotine dependence and reward differ between adolescent and adult male mice. J Pharmacol Exp Ther 322:399407. 
Koya E, Uejima JL, Wihbey KA, Bossert JM, Hope BT, Shaham Y (2009) Role of ventral medial prefrontal cortex in incubation of cocaine craving. Neuropharmacology 56:177-85.

Kujala UM, Kaprio J, Rose RJ (2007) Physical activity in adolescence and smoking in young adulthood: a prospective twin cohort study. Addiction 102:1151-1157.

Lecca D, Cacciapaglia F, Valentini V, Gronli J, Spiga S, Di Chiara G (2006) Preferential increase of extracellular dopamine in the rat nucleus accumbens shell as compared to that in the core during acquisition and maintenance of intravenous nicotine self-administration. Psychopharmacology (Berl ) 184:435-446.

Lett BT, Grant VL, Koh MT, Flynn G (2002) Prior experience with wheel running produces cross-tolerance to the rewarding effect of morphine. Pharmacology Biochemistry and Behavior 72:101-105.

Lett BT, Grant VL, Koh MT (2001) Naloxone attenuates the conditioned place preference induced by wheel running in rats. Physiol Behav 72: 355-358.

Lett BT, Grant VL, Byrne MJ, Koh MT (2000) Pairings of a distinctive chamber with the aftereffect of wheel running produce conditioned place preference. Appetite 34: $87-94$.

Levin ED, Slade S, Wells C, Cauley M, Petro A, Vendittelli A, Johnson M, Williams P, Horton K, Rezvani AH (2011) Threshold of adulthood for the onset of nicotine self-administration in male and female rats. Behav Brain Res 225:473-481. 
Levin ED, Lawrence SS, Petro A, Horton K, Rezvani AH, Seidler FJ, Slotkin TA (2007) Adolescent vs. adult-onset nicotine self-administration in male rats: duration of effect and differential nicotinic receptor correlates. Neurotoxicol Teratol 29:458465.

Levin ED, Rezvani AH, Montoya D, Rose JE, Swartzwelder HS (2003) Adolescent-onset nicotine self-administration modeled in female rats. Psychopharmacology (Berl ) 169:141-149.

Li S, Zou S, Coen K, Funk D, Shram MJ, Le AD (2012) Se differences in yohimbineinduced increases in the reinforcing efficacy of nicotine in adolescent rats. Addict Biol doi: 10.1111/j.1369-1600.2012.00473.x. [Epub ahead of print]

Li Y, Acerbo MJ, Robinson TE (2004) The induction of behavioural sensitization is associated with cocaine-induced structural plasticity in the core (but not shell) of the nucleus accumbens. Eur J Neurosci 20:1647-1654.

Liechti ME, Lhuillier L, Kaupmann K, Markou A (2007) Metabotropic glutamate 2/3 receptors in the ventral tegmental area and the nucleus accumbens shell are involved in behaviors relating to nicotine dependence. The Journal of Neuroscience 27:9077-9085.

Lisha NE, Sussman S (2010) Relationship of high school and college sports participation with alcohol, tobacco, and illicit drug use: A review. Addict Behav 35:399-407. 
Loweth JA, Tseng KY, Wolf ME (2013) Adaptations in AMPA receptor transmission in the nucleus accumbens contributing to incubation of cocaine craving. Neuropharmacology doi: 10.1016/j.neuropharm.2013.04.061. [Epub ahead of print]

Lu L, Grimm JW, Hope BT, Shaham Y (2004) Incubation of cocaine craving after withdrawal: a review of preclinical data. Neuropharmacology 47: 214-226.

Lynch WJ (2006) Sex differences in vulnerability to drug self-administration. Experimental and Clinical Psychopharmacology, 14: 34-41.

Lynch WJ (2008) Acquisition and maintenance of cocaine self-administration in adolescent rats: effects of sex and gonadal hormones. Psychopharmacology 197: 237-246.

Lynch WJ (2009) Sex and ovarian hormones influence vulnerability and motivation for nicotine during adolescence in rats. Pharmacol Biochem Behav 94: 43-50.

Lynch WJ, Peterson AB, Sanchez V, Abel J, Smith MA (2013) Exercise as a novel treatment for drug addiction: a neurobiological and stage-dependent hypothesis. Neuroscience \& Biobehavioral Reviews 37:1622-1644.

Lynch WJ, Piehl KB, Acosta G, Peterson AB, Hemby SE (2010) Aerobic exercise attenuates reinstatement of cocaine-seeking behavior and associated neuroadaptations in the prefrontal cortex. Biol Psychiatry 68: 774-777.

Lynch WJ, Roth ME, Carroll ME (2002) Biologicial basis of sex differences in drug abuse: preclinical and clinical studies Psychopharmacology (Berl) 164: 121-137. 
Lyness W, Friedle N, Moore K (1979) Destruction of dopaminergic nerve terminals in nucleus accumbens: effect on d-amphetamine self-administration. Pharmacology Biochemistry and Behavior 11:553-556.

Mameli M, Halbout B, Creton C, Engblom D, Parkitna JR, Spanagel R, Lüscher C (2009) Cocaine-evoked synaptic plasticity: persistence in the VTA triggers adaptations in the NAc. Nat Neurosci 12:1036-1041.

Matta SG, Balfour DJ, Benowitz NL et al. (2007) Guidelines on nicotine dose selection for in vivo research. Psychopharmacology 190: 269-319.

Mattila VM, Raisamo S, Pihlajamäki H, Mäntysaari M, Rimpelä A (2012) Sports activity and the use of cigarettes and snus among young males in Finland in 1999-2010. BMC Public Health 12:230.

McDonald C, Eppolito A, Brielmaier J, Smith L, Bergstrom H, Lawhead M, Smith R (2007) Evidence for elevated nicotine-induced structural plasticity in nucleus accumbens of adolescent rats. Brain Res 1151:211-218.

McDonald CG, Dailey VK, Bergstrom HC, Wheeler TL, Eppolito AK, Smith LN, Smith RF (2005) Periadolescent nicotine administration produces enduring changes in dendritic morphology of medium spiny neurons from nucleus accumbens. Neurosci Lett 385:163-167. 
McFarland K, Lapish CC, Kalivas PW (2003) Prefrontal glutamate release into the core of the nucleus accumbens mediates cocaine-induced reinstatement of drugseeking behavior. The Journal of neuroscience 23:3531-3537.

Meeusen R, Smolders I, Sarre S, De Meirleir K, Keizer H, Serneels M, Ebinger G, Michotte Y (1997) Endurance training effects on neurotransmitter release in rat striatum: an in vivo microdialysis study. Acta Physiol Scand 159:335-341.

Melis M, Spiga S, Diana M (2005) The dopamine hypothesis of drug addiction: hypodopaminergic state. Int Rev Neurobiol 63:101-154.

Melnick MJ, Miller KE, Sabo DF, Farrell MP, Barnes GM (2001) Tobacco use among high school athletes and nonathletes: Results of the 1997 Youth Risk Behavior Survey. Adolescence 36:727-747.

Miller ML, Vaillancourt BD, Wright MJ Jr, Aarde SM, Vandewater SA, Creehan KM, Taffe MA (2011) Reciprocal inhibitory effects of intravenous dmethamphetamine self-administration and wheel activity in rats. Drug Alcohol Depend 121: 90-6.

Moore MJ, Werch CE (2005) Sport and physical activity participation and substance use among adolescents. Journal of Adolescent Health 36:486-493.

Natividad LA, Buczynski MW, Parsons LH, Torres OV, O’Dell LE (2012) Adolescent rats are resistant to adaptations in excitatory and inhibitory mechanisms that 
modulate mesolimbic dopamine during nicotine withdrawal. J Neurochem 123: $578-588$.

Natividad LA, Tejeda HA, Torres OV, O'Dell LE (2010) Nicotine withdrawal produces a decrease in extracellular levels of dopamine in the nucleus accumbens that is lower in adolescent versus adult male rats. Synapse 64: 136-145.

Naylor AH, Gardner D, Zaichkowsky L (2001) Drug use patterns among high school athletes and nonathletes. Adolescence.

Nelson MC, Gordon-Larsen P (2006) Physical activity and sedentary behavior patterns are associated with selected adolescent health risk behaviors. Pediatrics 117:12811290.

Nimchinsky EA, Sabatini BL, Svoboda K (2002) Structure and function of dendritic spines. Annu Rev Physiol 64:313-353.

Nisell M, Nomikos GG, Svensson TH (1994) Systemic nicotine-induced dopamine release in the rat nucleus accumbens is regulated by nicotinic receptors in the ventral tegmental area. Synapse 16:36-44.

Norrholm S, Bibb J, Nestler E, Ouimet C, Taylor J, Greengard P (2003) Cocaine-induced proliferation of dendritic spines in nucleus accumbens is dependent on the activity of cyclin-dependent kinase-5. Neuroscience 116:19-22. 
O’Dell LE, Koob GF (2007) 'Nicotine deprivation effect' in rats with intermittent 23hour access to intravenous nicotine self-administration. Pharmacol Biochem Behav 86: 346-353.

Olsen CM, Winder DG (2012) Stimulus dynamics increase the self-administration of compound visual and auditory stimuli. Neurosci Lett 511: 8-11.

Palmatier MI, Liu X, Donny EC, Caggiula AR, Sved AF (2007) Metabotropic glutamate 5 receptor (mGluR5) antagonists decrease nicotine seeking, but do not affect the reinforcement enhancing effects of nicotine. Neuropsychopharmacology 33:21392147.

Parkinson JA, Olmstead MC, Burns LH, Robbins TW, Everitt BJ (1999) Dissociation in Effects of Lesions of the Nucleus Accumbens Core and Shell on Appetitive Pavlovian Approach Behavior and the Potentiation of Conditioned Reinforcement and Locomotor Activity byd-Amphetamine. The Journal of neuroscience 19:2401-2411.

Pastor Y, Balaguer I, Pons D, García-Merita M (2003) Testing direct and indirect effects of sports participation on perceived health in Spanish adolescents between 15 and 18 years of age. J Adolesc 26:717-730.

Pate RR, Trost SG, Levin S, Dowda M (2000) Sports participation and health-related behaviors among US youth. Arch Pediatr Adolesc Med 154:904. 
Pate RR, Heath GW, Dowda M, Trost SG (1996) Associations between physical activity and other health behaviors in a representative sample of US adolescents. Am J Public Health 86:1577-1581.

Paterson NE, Markou A (2004) Prolonged nicotine dependence associated with extended access to nicotine self-administration in rats. Psychopharmacology 173:64-72.

Paxinos G, Watson C (2005) The Rat Brain in Stereotaxic Coordinates (5th Edition). Elsevier Academic Press, San Diego.

Perkins KA, Scott J (2008) Sex differences in long-term smoking cessation rates due to nicotine patch. Nicotine Tobacco Res 10:1245-1251.

Perkins KA, Donny E, Caggiula AR (1999) Sex differences in nicotine effects and selfadministration: review of human and animal evidence. Nicotine Tob Res 1, 301315.

Peterson AB, Abel JM, Lynch WJ (2013) Dose-dependent effects of wheel running on cocaine-seeking and prefrontal cortex Bdnf exon IV expression in rats. Psychopharmacology (Berl ):1-10.

Piazza PV, Le Moal M (1998) The role of stress in drug self-administration. Trends Pharmacol Sci 19:67-74.

Pickens CL, Airavaara M, Theberge F, Fanous S, Hope BT, Shaham Y (2011) Neurobiology of the incubation of drug craving. Trends Neurosci 34:411-420. 
Pierce JP, Gilpin E (1996) How long will today's new adolescent smoker be addicted to cigarettes? Am J Public Health 86:253-256.

Piper ME, Cook JW, Schlam TR, Jorenby DE, Smith SS, Bolt DM, Loh W (2010) Gender, race, and education differences in abstinence rates among participants in two randomized smoking cessation trials. Nicotine Tobacco Res 12:647-657.

Pontieri F, Tanda G, Di Chiara G (1995) Intravenous cocaine, morphine, and amphetamine preferentially increase extracellular dopamine in the" shell" as compared with the" core" of the rat nucleus accumbens. Proceedings of the National Academy of Sciences 92:12304-12308.

Pontieri FE, Tanda G, Orzi F, Di Chiara G (1996) Effects of nicotine on the nucleus accumbens and similarity to those of addictive drugs.

Prescott E, Hippe M, Schnohr P, Hein HO, Vestbo J (1998) Smoking and risk of myocardial infarction in women and men: longitudinal population study. BMJ 316:1043.

Rainey CJ, McKeown RE, Sargent RG, Valois RF (1996) Patterns of tobacco and alcohol use among sedentary, exercising, nonathletic, and athletic youth. J Sch Health $66: 27-32$.

Redila V, Christie B (2006) Exercise-induced changes in dendritic structure and complexity in the adult hippocampal dentate gyrus. Neuroscience 137:1299-1307. 
Ridenour TA, Lanza ST, Donny EC, Clark DB (2006) Different lengths of times for progressions in adolescent substance involvement. Addict Behav 31:962-983.

Roberts D, Koob GF (1982) Disruption of cocaine self-administration following 6hydroxydopamine lesions of the ventral tegmental area in rats. Pharmacology Biochemistry and Behavior 17:901-904.

Roberts V, Maddison R, Simpson C, Bullen C, Prapavessis H (2012) The acute effects of exercise on cigarette cravings, withdrawal symptoms, affect and smoking behavior: systematic review update and meta-analysis. Psychopharmacology 222: $1-15$.

Robinson TE, Kolb B (2004) Structural plasticity associated with exposure to drugs of abuse. Neuropharmacology 47:33-46.

Robinson TE, Gorny G, Mitton E, Kolb B (2001) Cocaine self-administration alters the morphology of dendrites and dendritic spines in the nucleus accumbens and neocortex.

Robinson TE, Kolb B (1999) Alterations in the morphology of dendrites and dendritic spines in the nucleus accumbens and prefrontal cortex following repeated treatment with amphetamine or cocaine. Eur J Neurosci 11:1598-1604.

Robinson TE, Kolb B (1997) Persistent structural modifications in nucleus accumbens and prefrontal cortex neurons produced by previous experience with amphetamine. The Journal of neuroscience 17:8491-8497. 
Rozeske RR, Greenwood BN, Fleshner M, Watkins LR, Maier SF (2011) Voluntary wheel running produces resistance to inescapable stress-induced potentiation of morphine conditioned place preference. Behav Brain Res 219:378-381.

RTI International, United States of America, SAMHSA, Office of Applied Studies, United States of America (2010) A Day in the Life of American Adolescents: Substance Use Facts Update.

Russo SJ, Dietz DM, Dumitriu D, Morrison JH, Malenka RC, Nestler EJ (2010) The addicted synapse: mechanisms of synaptic and structural plasticity in nucleus accumbens. Trends Neurosci 33:267-276.

Sanchez V, Moore CF, Brunzell DH, Lynch WJ (2013a) Effect of wheel-running during abstinence on subsequent nicotine-seeking in rats. Psychopharmacology 227: 403-411.

Sanchez V, Moore CF, Brunzell DB, Lynch WJ (2013b) Sex differences in the effect of wheel running on subsequent nicotine-seeking in a rat adolescent-onset selfadministration model. Psychopharmacology (Berl) in press. DOI: 10.1007/s00213-013-3359-3

Sanchez V, Feinstein SD, Lunardi N, Joksovic PM, Boscolo A, Todorovic SM, JevtovicTodorovic V (2011) General anesthesia causes long-term impairment of mitochondrial morphogenesis and synaptic transmission in developing rat brain. Anesthesiology. 115: 992-1002. 
Schmidt HD, Pierce RC (2010) Cocaine-induced neuroadaptations in glutamate transmission. Ann N Y Acad Sci 1187:35-75.

Shaham Y, Shalev U, Lu L, de Wit H, Stewart J (2003) The reinstatement model of drug relapse: history, methodology and major findings. Psychopharmacology (Berl ) 168:3-20.

Shram MJ, Lê AD (2010) Adolescent male Wistar rats are more responsive than adult rats to the conditioned rewarding effects of intravenously administered nicotine in the place conditioning procedure. Behav Brain Res 206:240-244.

Shram MJ, Li Z, Lê AD (2008) Age differences in the spontaneous acquisition of nicotine self-administration in male Wistar and Long-Evans rats. Psychopharmacology (Berl) 197:45-58.

Shram MJ, Funk D, Li Z, Lê AD (2007) Nicotine self-administration, extinction responding and reinstatement in adolescent and adult male rats: evidence against a biological vulnerability to nicotine addiction during adolescence. Neuropsychopharmacology 33:739-748.

Shram MJ, Funk D, Li Z, Lê AD (2006) Periadolescent and adult rats respond differently in tests measuring the rewarding and aversive effects of nicotine. Psychopharmacology (Berl ) 186:201-208. 
Smith MA, Pennock MM, Walker KL, Lang KC (2012) Access to a running wheel decreases cocaine-primed and cue-induced reinstatement in male and female rats. Drug Alcohol Depend 121:54-61.

Smith MA, Pitts EG (2012) Wheel running decreases the positive reinforcing effects of heroin. Pharmacological Reports 64:960-964.

Smith MA, Witte MA (2012) The effects of exercise on cocaine self-administration, food-maintained responding, and locomotor activity in female rats: Importance of the temporal relationship between physical activity and initial drug exposure. Exp Clin Psychopharmacol 20:437.

Smith MA, Pitts EG (2011) Access to a running wheel inhibits the acquisition of cocaine self-administration. Pharmacol Biochem Behav 100: 237-43.

Smith MA, Walker KL, Cole KT, Lang KC (2011) The effects of aerobic exercise on cocaine self-administration in male and female rats. Pyschopharmacology 218: 357-69.

Smith MA, Schmidt KT, Iordanou JC, Mustroph ML (2008) Aerobic exercise decreases the positive-reinforcing effects of cocaine. Drug Alcohol Depend 98: 129-135.

Solinas M, Chauvet C, Thiriet N, El Rawas R, Jaber M (2008) Reversal of cocaine addiction by environmental enrichment. Proceedings of the National Academy of Sciences 105:17145-17150. 
Stranahan AM, Khalil D, Gould E (2007) Running induces widespread structural alterations in the hippocampus and entorhinal cortex. Hippocampus 17:10171022.

Substance Abuse and Mental Health Services Administration (2013) Results from the 2012 National Survey on Drug Use and Health: Summary of National Findings, NSDUH Series H-46, HHS Publication No. SMA 13-4795. Rockville, MD.

Substance Abuse and Mental Health Services Administration (2010) Results from the 2009 National Survey on Drug Use and Health: Volume I. Summary of National Findings (Office of Applied Studies, NSDUH Series H-38A, HHS Publication No. SMA 10-4856Findings). Rockville, MD.

Taioli E, Wynder EL (1991) Effect of the age at which smoking begins on frequency of smoking in adulthood. N Engl J Med 325:968-969.

Takumi Y, Ramírez-León V, Laake P, Rinvik E, Ottersen OP (1999) Different modes of expression of AMPA and NMDA receptors in hippocampal synapses. Nat Neurosci 2:618-624.

Tanski SE, Prokhorov AV, Kein JD (2004) Youth and tobacco. Minerva Pediatr 56: 553565.

Taylor A, Katomeri M (2007) Walking reduces cue-elicited cigarette cravings and withdrawal symptoms, and delays ad libitum smoking. Nicotine Tob Res 9: 11831190. 
Taylor H, Ussher MH, Faulkner (2007) The acute effects of exercise on cigarette cravings, withdrawal symptoms, affect and smoking behavior: a systematic review. Nicotine Tob Res 9: 1183-1190.

Tejeda HA, Natividad LA, Orfila JE, Torres OV, O’Dell LE (2012) Dysregulation of kappa-opioid receptor systems by chronic nicotine modulate the nicotine withdrawal syndrome in an age dependent manner. Pyschopharmacology 224: 289-301.

Terry-McElrath YM, O'Malley PM (2011) Substance use and exercise participation among young adults: parallel trajectories in a national cohort-sequential study. Addiction 106:1855-1865.

Tessari M, Pilla M, Andreoli M, Hutcheson DM, Heidbreder CA (2004) Antagonism at metabotropic glutamate 5 receptors inhibits nicotine-and cocaine-taking behaviours and prevents nicotine-triggered relapse to nicotine-seeking. Eur J Pharmacol 499:121-133.

Thanos PK, Stamos J, Robinson LS, Heyman G, Tucci A, Wang GJ, Robinson JK, Anderson BJ, Volkow ND (2013) Daily treadmill exercise attenuates cocaine cueinduced reinstatement and cocaine induced locomoter responses but increases cocaine-primed reinstatement. Behav Brain Res 239: 8-14. 
Thanos PK, Tucci A, Stamos J, Robison L, Wang G, Anderson BJ, Volkow ND (2010) Chronic forced exercise during adolescence decreases cocaine conditioned place preference in Lewis rats. Behav Brain Res 215:77-82.

Thorner ED, Jaszyna-Gasior M, Epstein DH, Moolchan ET (2007) Progression to daily smoking: Is there a gender difference among cessation treatment seekers? Subst Use Misuse 42:829-835.

Torrella TA, Badanich KA, Philpot RM, Kerstein CL, Wecker L (2004) Developmental differences in nicotine place conditioning. Ann N Y Acad Sci 1021:399-403.

Torres OV, Tejeda HA, Natividad LA, O’Dell LE (2008) Enhanced vulnerability to the rewarding effects of nicotine during the adolescent period of development. Pharmacol Biochem Behav 90: 658-653.

Torres OV, Natividad LA, Tejeda HA, Van Weelden SA, O’Dell LE (2009) Female rats display dose-dependent differences to the rewarding and aversive effects of nicotine in an age-, hormone-, and sex-dependent manner. Psychopharmacology 206: 303-312.

Ussher MH, Taylor A, Faulkner G (2012) Exercise interventions for smoking cessation. Cochrane Database of Systematic Reviews Issue 1. Art. No.: CD002295. DOI: 10.1002/14651858.CD002295.pub4.

Valentine JD, Hokanson JS, Matta SG, Sharp BM (1997) Self-administration in rats allowed unlimited access to nicotine. Psychopharmacology 133: 300-304. 
Van De Ven M, Greenwood P, Engels R, Olsson C, Patton G (2010) Patterns of adolescent smoking and later nicotine dependence in young adults: a 10-year prospective study. Public Health 124:65-70.

Vastola BJ, Douglas LA, Varlinskaya EI, Spear LP (2002) Nicotine-induced conditioned place preference in adolescent and adult rats. Physiol Behav 77:107-114.

Voorn P, Vanderschuren LJ, Groenewegen HJ, Robbins TW, Pennartz C (2004) Putting a spin on the dorsal-ventral divide of the striatum. Trends Neurosci 27:468-474.

Werch C, Moore MJ, DiClemente, CC, Bledsoe R, Jobli E (2005) A multihealth behavior intervention integrating physical activity and substance use prevention for adolescents. Prev Sci 6: 213-226.

Wissman AM, May RM, Woolley CS (2012) Ultrastructural analysis of sex differences in nucleus accumbens synaptic connectivity. Brain Structure and Function 217:181190.

Wolf ME, Ferrario CR (2010) AMPA receptor plasticity in the nucleus accumbens after repeated exposure to cocaine. Neuroscience \& Biobehavioral Reviews 35:185211.

World Health Organization (2003) World health report: Shaping the future. http://www.who.int/whr/2003/en/, retrieved 8 Aug 2012.

World Health Organization. WHO Report on the Global Tobacco Epidemic, 2009. Geneva: World Health Organization, 2008 [accessed 2013 March 9]. http://www.who.int/tobacco/mpower/2009/a2_gtcr_report_summary.pdf 
Zang EA, Wynder EL (1996) Differences in lung cancer risk between men and women: examination of the evidence. J Natl Cancer Inst 88:183-192.

Zlebnik NE, Anker JJ, Gliddon LA, Carroll ME (2010) Reduction of extinction and reinstatement of cocaine seeking by wheel running in females. Pyschopharmacology 209: 113-125.

Zlebnik NE, Anker JJ, Carroll ME (2012) Exercise to reduce the escalation of cocaine self-administration in adolescent and adult rats. Pyschopharmacology Published online: 03 July 2012. PMID 22752381. 


\section{APPENDIX}

\section{General anesthesia causes long-term impairment of mitochondrial morphogenesis and synaptic transmission in \\ developing rat brain}

\section{Abstract}

Background: Clinically used general anesthetics, alone or in combination, are damaging to the developing mammalian brain. In addition to causing widespread apoptotic neurodegeneration in vulnerable brain regions, exposure to general anesthesia at the peak of synaptogenesis causes learning and memory deficiencies later in life. Our in-vivo rodent studies have suggested that activation of the intrinsic (mitochondria-dependent) apoptotic pathway is the earliest warning sign of neuronal damage, suggesting that a disturbance in mitochondrial integrity and function could be the earliest triggering events.

Methods: Since proper and timely mitochondrial morphogenesis is critical for brain development, we examined the long-term effects of a commonly used anesthesia combination (isoflurane, nitrous oxide, and midazolam) on the regional distribution, ultrastructural properties, and electron transport chain function of mitochondria, as well as synaptic neurotransmission, in the subiculum of rat pups.

Results: We found that this anesthesia, administered at the peak of synaptogenesis, causes protracted injury to mitochondria, including significant 
enlargement of mitochondria, impairment of their structural integrity, increase in their complex IV activity and decrease in their regional distribution in presynaptic neuronal profiles where their presence is crucially important for the normal development and functioning of synapses. Consequently, we showed that impaired mitochondrial morphogenesis is accompanied by heightened autophagic activity, decreased mitochondrial density and long-lasting disturbances in inhibitory synaptic neurotransmission. The interrelation of these phenomena remains to be established.

Conclusion: We suggest that developing mitochondria are exquisitely vulnerable to general anesthesia and, accordingly, may be important early target of anesthesia-induced developmental neurodegeneration. 


\section{Introduction}

Over the last decade, a myriad of studies have presented evidence that various mammalian species are susceptible to significant neurotoxicity when exposed to general anesthesia during early stages of their brain development. Anesthesia-induced neurotoxicity is described as apoptotic and its severity is age-dependent; i.e., the peak of susceptibility coincides with the peak of synaptogenesis. ${ }^{1}$

Since it has been suggested that general anesthetics used in clinical practice are not as innocuous as they were previously thought to be, ${ }^{2}$ recent research has been focused on deciphering the earliest cellular targets and mechanistic pathways of anesthesia-induced developmental neurotoxicity so that a method of effective and timely prevention can be devised. This is essential because the use of general anesthetics often cannot be avoided when a child's life is in danger.

Based on presently available evidence, it appears that very early events in anesthesia-induced apoptotic neurodegeneration involve the activation of a mitochondria-dependent apoptotic cascade, ${ }^{1,3}$ which leads to effector caspase activation and DNA fragmentation. Thus, mitochondria may be the initial and most vulnerable target of anesthesia-induced impairment of neuronal development. Since mitochondria are crucially important organelles for the formation, maintenance, and function of developing synapses, which can be permanently impaired by a single exposure to anesthesia, ${ }^{4,5}$ we did a series of 
experiments on the effects of general anesthesia on morphogenesis and regional distribution of mitochondria in the developing subiculum.

We found that early exposure to general anesthesia significantly modulates mitochondrial morphogenesis and the function of mitochondria electron transport chain enzyme activity. Regional distribution of mitochondria in presynaptic neuronal profiles is also significantly reduced, where their presence is crucially important for the normal development and function of synapses. We also found that impaired mitochondrial morphogenesis is accompanied by heightened autophagic activity, protracted neuropil destruction, and long-lasting disturbances in inhibitory synaptic neurotransmission. 


\section{Materials and Methods}

Animals. We used Sprague-Dawley rat pups (Harlan Laboratories, Indianapolis, IN) at postnatal day (P) 7 for all experiments, since this is when they are most vulnerable to anesthesia-induced neuronal damage ${ }^{1}$. Experimental rat pups were exposed to 6 hours of anesthesia; controls were exposed to 6 hours of mock anesthesia (vehicle). After the administration of anesthesia, rats were allowed to recover and were reunited with their mothers. Each day, we weighed them and noted their general appearance. On P21, these rats were randomly divided into three groups, one for ultrastructural analysis of the subiculum, one for measuring electron transport chain enzyme activity, and the other for functional studies of synaptic activity (patch-clamp recordings of excitatory and inhibitory synaptic neurotransmission). Rats were used at P21 for ultrastructural and enzyme activity examination and at P21-P28 for electrophysiological studies, since synaptic maturation is generally completed at this age.

All experiments were approved by the Animal Use and Care Committee of the University of Virginia Health System, Charlottesville, VA and were done in accordance with the Public Health Service's Policy on Human Care and Use of Laboratory Animals. Efforts were made to minimize the number of animals used.

Anesthesia. Nitrous oxide and oxygen were delivered using a calibrated flowmeter. To administer a specific concentration of nitrous oxide/oxygen and isoflurane in a highly controlled environment, an anesthesia chamber was 
used. $^{1,3,6}$ Isoflurane was administered using an agent-specific vaporizer that delivers a set percentage of anesthetic into the anesthesia chamber. Midazolam (Sigma-Aldrich Chemical, St. Louis, MO) was dissolved in $0.1 \%$ dimethyl sulfoxide just before administration. For control animals, $0.1 \%$ dimethyl sulfoxide was used alone. To administer a specific concentration of nitrous oxide/oxygen and isoflurane in a highly controlled environment, an anesthesia chamber was used ${ }^{1,3,6}$. Rats were kept normothermic throughout the experiment, as previously described. ${ }^{7}$ For control experiments, air was substituted for the gas mixture. After initial equilibration of the nitrous oxide loxygen/isoflurane or air atmosphere inside the chamber, the composition of the chamber gas was analyzed by infrared analyzer (Datex Ohmeda, Madison, WI) to establish the concentrations of nitrous oxide or nitrogen, isoflurane, carbon dioxide, and oxygen. We used our standard general anesthesia protocol, giving P7 rat pups a single injection of midazolam ( $9 \mathrm{mg} / \mathrm{kg}$, intraperitoneally) followed by 6 hours of nitrous oxide $(75 \%)$, isoflurane $(0.75 \%)$, and oxygen (approximately $24 \%$ ). Several studies have shown that this protocol causes severe neurodegenerative damage to developing neurons $s^{1,3,5,6}$.

Histopathological studies. On P21, each pup was deeply anesthetized with phenobarbital (65 mg/kg, intraperitoneally) (University of Virginia Pharmacy, Charlottesville, VA). After cannulating the left ventricle, we clamped the descending aorta and did an initial flush with Tyrodes solution (30-40 ml) (SigmaAldrich Chemical). For morphometric analyses of the neuropil, this was followed 
by 10 min of continuous perfusion with freshly prepared paraformaldehyde (4\%) and glutaraldehyde $(0.5 \%) .{ }^{1,3,6,8}$ For morphometric analyses of pyramidal neurons, perfusion was done using paraformaldehyde (2\%) and glutaraldehyde $(2 \%)$. After the perfusion, we removed the rats' brains and stored them in the same fixative overnight. Both control and experimental pups were perfused by an experienced experimenter on the same day, using the same solution to assure uniform tissue fixation. Any brains considered to have been inadequately perfused were not processed for electron microscopy (EM) analysis. Fixed brains were coronally sectioned (50-75 $\mu$ m thick) with a DTK-1000 microslicer (Ted Pella, Tools for Science and Industry, Redding CA). The subiculum was localized as described in anatomical maps, ${ }^{9}$ fixed in $2 \%$ osmium tetroxide (Electron Microscopy Sciences, Hatfield, PA), stained with 4\% uranyl acetate (Electron Microscopy Sciences, Hatfield, PA), and embedded in aclar sheets using epon-araldite resins. The subiculum was then dissected from the aclar sheets and embedded in BEEM ${ }^{\mathrm{TM}}$ capsules (Electron Microscopy Sciences, Hatfield, PA). To prepare capsules for microtome cutting (Sorvall MT-2 microtome, Ivan Sorvall, Norwalk, CT) the tips were manually trimmed so that ultrathin slices (silver interference color, 600-900 A) could be cut using a diamond knife (Diatome, Hatfield, PA). Ultrathin sections were placed on grids and examined using a 1230 TEM electron microscope (Carl Zeiss, Oberkochen, Germany). Using a 16M-pixel digital camera (SIA-12C digital cameras, Scientific Instruments and Applications, Duluth, GA), we took 12 random, nonoverlapping 
electron micrographs $(12,000 X$ magnification) of each subicular layer (pyramidal, polymorphic, and molecular). Our electron micrographs depicted neuropils and large pyramidal neurons, depending on the type of analysis needed (see Results). The investigator analyzing electron micrographs was blinded to the experimental conditions.

Morphometric analyses. To do morphometric analyses of mitochondria in the cytoplasmic soma of pyramidal neurons in their entirety which is not possible to perform with a single photo frame at such high magnification (12,000x) we took multiple sequential pictures and tiled them seamlessly together to make a mosaic of one whole cell body ( $\mathrm{n}=15$ neurons in control group and anesthesiatreated group each). From these mosaic pictures, the cytoplasm area and mitochondrial area were measured using Image-Pro Plus 6.1 computer software (MediaCybernetics, Bethesda, MD). The number of animals used for detailed ultrastructural histological studies was sufficiently powered to make proper conclusions. $^{5}$

For morphometric analyses of mitochondria in presynaptic neuronal profiles, we first identified the synapses, using the following criteria: the presence of a postsynaptic density; the presence of more than one synaptic vesicle closely apposed to the presynaptic membrane; and the presence of a synaptic cleft delineated by parallel pre- and postsynaptic membranes. ${ }^{5,10}$ Both excitatory and inhibitory synapse-bearing presynaptic neuronal profiles were examined using the criteria specified by Crain et al. ${ }^{11}$ Once identified, the area of the presynaptic 
neuronal profile was measured and the presence or absence of mitochondria was noted. Using Image-Pro Plus 6.1 computer software, the area of mitochondria, where present, were measured so that the mitochondrial index could be calculated; the mitohondrial index is defined as the ratio between mitochondrial and presynaptic neuronal profile areas.

Spectrophotometric analyses of mitochondrial electron transport chain activity. After homogenizing and centrifuging subicular tissue, we measured mitochondrial electron transport chain (ETC) and citrate synthase (CS) activity using a Genesis 10-UV spectrophotometer (Thermo, Rochester, NY). The incubation temperatures for complexes I, II, and CS were $30^{\circ} \mathrm{C}$ and, for complex IV, $38^{\circ} \mathrm{C}$. Electron transport chain (ETC) complex activities were determined in supernatants as described by Perez-Carreras et al. ${ }^{12}$ and expressed as a percentage of the specific activity of CS to correct for the neuronal content of mitochondria. ETC assays were performed in triplicates.

Statistical analysis: Single comparisons among groups were made using unpaired two-tailed t-test. When ANOVAs with repeated measures were needed, the Bonferroni correction was used to help maintain prescribed alpha levels (e.g. 0.05). Using the standard version of GraphPad Prism 5.01 software (Media Cybernetics, Inc., Bethesda, MD) we considered $p<0.05$ to be statistically significant. All the data are presented as mean \pm standard error of mean (SEM).

Electrophysiology studies: All experiments were done on 300- $\mu \mathrm{m}$-thick transverse rat brain slices from 21-28 day-old animals using procedures 
described previously. ${ }^{13}$ The subiculum was localized as described in anatomical maps. $^{9}$

Rats were briefly anesthetized with isoflurane and decapitated. Their brains were rapidly removed and placed in chilled $\left(4^{\circ} \mathrm{C}\right)$ cutting solution consisting, in $\mathrm{mM}$, of $2 \mathrm{CaCl}_{2}, 260$ sucrose, $26 \mathrm{NaHCO}_{3}, 10$ glucose, $3 \mathrm{KCl}, 1.25$ $\mathrm{NaH}_{2} \mathrm{PO}_{4}$, and $2 \mathrm{MgCl}_{2}$ equilibrated with a mixture of $95 \%$ oxygen and $5 \%$ carbon dioxide. A block of tissue containing the subiculum and hippocampus was glued to the chuck of a vibratome (World Precision Instruments, Sarasota, FL) and slices were cut in a transverse plane. The slices were incubated at $36^{\circ} \mathrm{C}$ in oxygenated saline for 1 hour, then placed in a recording chamber that was perfused with extracellular saline at a rate of $1.5 \mathrm{ml} / \mathrm{min}$. Incubating saline consisted, in mM, of $124 \mathrm{NaCl}, 4 \mathrm{KCl}, 26 \mathrm{NaHCO}_{3}, 1.25 \mathrm{NaH}_{2} \mathrm{PO}_{4}, 2 \mathrm{MgCl}_{2}, 10$ glucose, and $2 \mathrm{CaCl}_{2}$ equilibrated with a mixture of $95 \%$ oxygen and $5 \%$ carbon dioxide. Slices were maintained in the recording chamber at room temperature and remained viable for at least 1 hour. Since the half-life of halogenated volatile anesthetics in nerve tissue after the induction of anesthesia is only about 10 $\min ,{ }^{14}$ it is unlikely that the isoflurane used to euthanize the rats could have interfered with the results of our experiments, which were performed at least 2 hours later.

Recording procedures: Whole-cell recordings were obtained from pyramidal subiculum neurons visualized with an IR DIC camera (Hamamatsu 
C2400, Bridgewater, NJ) on a Zeiss 2 FS Axioscope (Carl Zeiss Jena, Thuringia, Germany) with a $40 \mathrm{X}$ lens.

The standard extracellular saline for recording of evoked inhibitory postsynaptic currents (eIPSCs) and evoked excitatory postsynaptic currents (eEPSCs) consisted, in $\mathrm{mM}$, of $2 \mathrm{CaCl}_{2}, 130 \mathrm{NaCl}, 1 \mathrm{MgCl}_{2}, 10$ glucose, 26 $\mathrm{NaHCO}_{3}, 1.25 \mathrm{NaH}_{2} \mathrm{PO}_{4}$, and $2 \mathrm{mM} \mathrm{KCl}$. This solution was equilibrated with a mixture of $95 \%$ oxygen and $5 \%$ carbon dioxide for at least 30 min and had a resulting $\mathrm{pH}$ of about 7.4. For recording elPSCs, we used an internal solution containing, in $\mathrm{mM}, 130 \mathrm{KCl}, 4 \mathrm{NaCl}, 0.5 \mathrm{CaCl}_{2}, 5$ ethylene glycol tetraacetic acid (EGTA), 10 4-(2-hydroxyethil)-1-piperazineethanesulfonic acid (HEPES), 2 $\mathrm{MgATP}_{2}, 0.5$ Tris-GTP, and 5 lidocaine $\mathrm{N}$-ethyl bromide. $\mathrm{pH}$ was adjusted with $\mathrm{KOH}$ to 7.25 . For recordings of eEPSCS, this solution was modified by replacing $\mathrm{KCl}$ with equimolar K-gluconate. To eliminate glutamatergic excitatory currents, all recordings of elPSCs were done in the presence of $5 \mu \mathrm{M} N B Q X(2,3-$ dihydroxy-6-nitro-7-sulfamoyl-benzo[f]quinoxaline-2,3-dione), $50 \mu \mathrm{M}$ d-APV (2R)amino-5-phosphonovaleric acid; AP5, (2R)-amino-5 phosphonopentanoate) in a bath solution. To eliminate inhibitory currents, all recordings of eEPSCs were done in the presence of $20 \mu \mathrm{M}$ picrotoxin [a noncompetitive gamma-amino butyric acid $(\mathrm{GABA})_{\mathrm{A}}$ antagonist] in the bath solution.

Synaptic stimulation of pyramidal subiculum neurons was achieved with a Constant Current Isolated Stimulator DS3 (Digitimer, Welwyn Garden City Hertfordshire, England). Electrical field stimulation was achieved by placing a 
stimulating electrode within the hippocampal CA1 soma layer with stimulation intervals of at least $20 \mathrm{sec}$ to allow recovery of synaptic responses. In all recordings, we first determined the current-output relationship, and then used current intensities in the stimulating electrode corresponding to the maximal amplitudes of elPSCs and eEPSCs. Recordings were made with the standard whole-cell voltage clamp technique. Electrodes having final resistances of 2-4 $\mathrm{M} \Omega$ were fabricated from thin-walled microcapillary glass. Membrane currents were recorded with an Axoclamp 200B amplifier (Molecular Devices, Foster City, CA). Voltage commands and digitization of membrane currents were done with Clampex 8.2 of the pClamp software package (Molecular Devices) running on an IBM-compatible computer (Dell, Inc., Round Rock, TX). Neurons were typically held at $-70 \mathrm{mV}$.

Analysis of current: Current waveforms or extracted data were fitted using Clampfit 8.2 (Molecular Devices) and Origin 7.0 (OriginLab, Northhampton, MA). The decay of elPSCs was estimated by a single-exponential term. All salts and chemicals were obtained from Sigma-Aldrich Chemical. 


\section{Results}

\section{General anesthesia disturbs the fine ultrastructural balance of developing} neuronal mitochondria

Since mitochondrial ultrastructure dictates their function, ${ }^{15}$ we studied the ultrastructural appearance of mitochondria with special emphasis on changes indicative of distorted mitochondrial integrity. Our work has been focused on the subiculum because this brain region is highly vulnerable to anesthesia-induced developmental neurodegeneration, as shown by substantial acute neuroapoptotic damage after the administration of anesthesia ${ }^{8,16}$ and chronic changes marked by substantial neuronal loss. ${ }^{16,17}$ The subiculum is part of the hippocampus proper and part of Papez's circuit; it is intertwined with the hippocampal CA1 region, anterior thalamic nuclei, and both the entorhinal and cingulate cortices. Accordingly, the subiculum is important in cognitive development, especially the development of learning and memory. ${ }^{18}$

For ultrastuctural analysis of mitochondria, we examined the perikaryon of pyramidal subicular neurons (Fig. 1A, B) and nerve terminals in subicular neuropil (Fig. 1C, D) two weeks after exposure to anesthesia (at P21, see Materials and Methods). We noted that in control subiculi (Fig. $1 \mathrm{~A}$ and $\mathrm{C}$ ), the mitochondria appear normal with no evidence of swelling or injury; there was a typical homogeneous staining pattern of the matrix without excessive pallor. In contrast, in the experimental subiculum (Fig. 1 B and D) many mitochondria 
displayed structural disorganization of cristae (see asterisks), including dilated intracristal spaces with vacuoles and overall swelling, which made the mitochondria appear substantially larger than normal. In addition, multiple mitochondrial profiles were suggestive of severe degenerative changes (Fig. $1 \mathrm{D}$, black arrows).

Since degenerated organelles are removed by autophagy, we investigated whether the use of general anesthesia leads to heightened autophagic activity. We examined pyramidal neurons in control and experimental subiculi for ultrastructural signs of autophagosomes, lysosomes, and autophagic vacuoles. Using electron microscopy as the gold standard to examine the formation of autophagic profiles, we found that experimental neurons displayed many autophagic profiles (Fig. 2A and 2B). Dispersed throughout the cytoplasm were many lysosomes (Fig. 2A, double asterisks) and autophagic vacuoles. These single-membrane structures are formed by the fusion of lysosomes and autophagosomes to allow the digestion of biological "garbage"; they are also referred to as autolysosomes (Fig. 2A, arrowheads). The morphological hallmark of autophagy is the formation of double-layered membrane structures called autophagosomes. Experimental neurons frequently contained multiple autophagosomes where parts of cannibalized mitochondria could be detected (Fig. 2B, single asterisk). The presence of numerous autophagic profiles suggests that general anesthesia increases the autophagic load in immature neurons. 
Since general anesthesia appears to cause mitochondrial enlargement (Fig. 1 B and D), we did a detailed morphometric analysis of mitochondria in the somas of pyramidal subicular neurons. When measured as a percent of the cytoplasmic area of pyramidal neurons, we found that mitochondria in the experimental neurons occupied approximately twice as much area of the cytoplasmic soma area than did controls $\left(22.5 \pm 3.1 \%\right.$ versus $13.44 \pm 1.2 \%,{ }^{*} p<$ 0.05) (Fig. 3A, $n=15$ neurons per group from 3 control and 3 experimental pups). This did not appear to be due to an increase in mitochondrial number, since mitochondrial density, presented as the number of mitochondria per unit area $\left(\mu \mathrm{m}^{2}\right)$ of cytoplasmic soma, was significantly lower in experimental pyramidal neurons as compared to control pyramidal neurons (Fig. 3B).

We confirmed that anesthesia causes mitochondrial enlargement when we categorized mitochondria as small (up to $0.05 \mu \mathrm{m}^{2}$ ), medium $\left(0.06\right.$ to $0.25 \mu \mathrm{m}^{2}$ ), large $\left(0.26\right.$ to $0.65 \mu \mathrm{m}^{2}$ ), and extra-large (above $0.65 \mu \mathrm{m}^{2}$ ) (Fig. 4). Indeed, we found a complete reversal of the ratio between small and large mitochondria in control and experimental animals. For example, about $15 \%$ of the total mitochondria in control pyramidal neurons were small ones, whereas only $5 \%$ of mitochondria in experimental neurons were small $\left.{ }^{* * *}, p<0.001\right)$ (Fig. 4A). Large mitochondria constituted only $5 \%$ of the total mitochondria in control animals, while more than $15 \%$ of the mitochondria in experimental pyramidal neurons were large $\left({ }^{*}, p<0.05\right)($ Fig. $4 C)$. Interestingly, although extra-large mitochondria represent less than $1 \%$ of the total number of mitochondria, they 
appeared to be two-fold more prevalent in experimental pyramidal neurons (Fig. 4D) than they were in control pyramidal neurons, suggesting that anesthesia causes a substantial imbalance in mitochondrial size, with a clear tendency toward mitochondrial enlargement $(n=15$ neurons per group from 3 control and 3experimental pups).

\section{General anesthesia disturbs regional distribution of developing neuronal} mitochondria

The proper development and function of synapses depends on mitochondrial support, since synaptogenesis has high metabolic requirements. ${ }^{19-}$ ${ }^{22}$ Therefore, regional distribution of mitochondria in presynaptic nerve terminals and their strategic placement in the vicinity of developing synapses is crucially important. Having demonstrated that general anesthesia causes substantial enlargement of mitochondria in neuronal somas, as shown in Figures 3 and 4, we then examined whether similar morphometric changes could be detected in presynaptic neuronal terminals. To compare regional distribution of mitochondria in presynaptic neuronal profiles in experimental and control subiculi, we quantified the number of presynaptic neuronal profiles, out of all those present in any given electron microscopy photo frame of subicular neuropils (at 12,000x magnification), that contained mitochondrial profiles. We expressed the findings as a percentage of presynaptic profiles containing mitochondria. We found a significantly higher $\left({ }^{*}, p<0.05\right)$ percentage of mitochondria-containing presynaptic profiles in control as compared to experimental subiculi (Fig. 5) ( $n=$ 
28 photo frames/group from 4 control and 4 experimental pups from two different litters).

To further assess our ultrastructural observation suggesting substantial swelling of mitochondrial profiles in presynaptic neuron terminals (Fig. 1D), we did detailed morphometric analysis of the area of mitochondrial profiles (in $\mu \mathrm{m}^{2}$ ). We found that experimental mitochondrial profiles were, on average, $38 \%$ larger than control mitochondrial profiles $\left({ }^{*}, p<0.05\right)($ Fig. $6 A)(n=30$ photo frames obtained from 5 control pups; $n=45$ photo frames obtained from 5 experimental pups; control and experimental pups were litter-matched; total of three different litters were used). When the areas of mitochondria-containing presynaptic nerve terminals were measured, we found no difference between control and experimental animals (Fig. 6B) $(n=30$ photo frames obtained from 5 control pups; $n=45$ photo frames obtained from 5 experimental pups; control and experimental pups were litter-matched; total of three different litters were used). Consequently, when we calculated the mitochondrial index, which is the ratio between mitochondrial area and mitochondria-containing presynaptic area, we found that this index was significantly higher in experimental subicular neuropils $\left({ }^{*}, \mathrm{p}<0.05\right)$ indicating that terminally distributed mitochondria display morphometric changes that are similar to those in mitochondria located in the soma (Fig. 6C). 
III. General anesthesia acutely disturbs functional balance in developing neuronal mitochondria

Since we have previously reported that general anesthesia causes acute disturbances of cytochrome $\mathrm{c}$ homeostasis within the first 4 hours of anesthesia exposure, ${ }^{1}$ we question whether anesthesia modulates the function of electron transport chain (ETC) complexes, particularly complex IV (cytochrome c oxidoreductase). Complex IV is of interest for three reasons: it depends on the availability of cytochrome c, since it transfers electrons from cytochrome $\mathrm{c}$ to oxygen, thus controlling the final steps of electron transport and adenosine triphosphate (ATP) synthesis; it is encoded in part by mitochondrial deoxyribonucleic acid, which makes it particularly vulnerable to mitochondrial dysfunction; modulation of complex IV activity has been shown to cause elevated free oxygen radical production ${ }^{23}$ thus making neurons vulnerable to excessive lipid peroxidation and protein oxidation. We measured complex IV activity in mitochondrial homogenate prepared from fresh subicular tissue of rat pups on P8, 24 hours after anesthesia treatment. Since citrate synthase (CS) activity is directly proportional to mitochondrial content, the activity of complex IV was expressed as a ratio (per the activity of citrate synthase). As shown in Figure 7A, there was a significant increase in complex IV activity 24 hours after anesthesia treatment $\left({ }^{*}, p<0.05\right)(n=8$ pups in control group; $n=6$ pups in experimental group). When we measured the activity of complexes I and II/III, we found no change in experimental groups as compared to controls (Fig. 7B, C, respectively) 
( $n=5$ pups in control group; $n=5$ pups in experimental group for complex I activity; $n=3$ in control group; $n=4$ pups in experimental group for complex II/III activity).

\section{General anesthesia impairs developmental synaptic transmission}

Given the fact that general anesthesia impairs synapse formation during early brain development, ${ }^{4,5}$ as well as our findings suggesting that general anesthetics impair mitochondrial morphogenesis and decrease the numbers of mitochondria in presynaptic neuronal profiles, we ask whether the diminished presence of mitochondria in anesthesia-treated subiculum has any bearing on the functional integrity of its synapses. To address this question, we examined inhibitory (eIPSC) and excitatory (eEPSC) synaptic transmission by recording from the pyramidal layer of control and anesthesia-treated rat subicular slices (see Methods). The traces in Figure 8A show representative elPSCs from the control (black line) and experimental group (gray line), both of which received anesthesia at P7. Paired stimulation of afferent fibers resulted in pair-pulse depression, a highly characteristic finding for subicular neurons (Fig. 8A). This test is done by analyzing changes in the ratio of elPSCs elicited by two identical presynaptic stimuli delivered in rapid succession. This paired-pulse depression of test elPSCs $(\mathrm{P} 2)$ relative to conditioning elPSCs $(\mathrm{P} 1)$ is thought to be due to depletion of a fraction of readily available synaptic sites. 
In comparison to the control group, the experimental group had $\sim 49 \%$ decrease in net charge transfer of elPSCs measured as the area under the curve $(p<0.05)$; a decreased decay time constant (tau) from $58 \pm 9 \mathrm{~ms}(n=14)$ to $34 \pm$ $4 \mathrm{~ms}(\mathrm{n}=12, \mathrm{p}<0.05)$; and a significantly altered paired-pulse ratio P2/P1 (amplitude of elPSC-2: amplitude of elPSC-1) from $0.81 \pm 0.02(n=14)$ to $0.87 \pm$ $0.01(n=12, p<0.05)$. The data are summarized in the histogram in Figure 8B.

It is generally accepted that presynaptic depressants that change the probability of transmitter release from presynaptic terminals cause a smaller fraction of the readily releasable pool of vesicles to undergo exocytosis and therefore decrease pair-pulse depression. ${ }^{24}$ This means that in the presence of presynaptic modulators, the ratio $\mathrm{P} 2: \mathrm{P} 1$ becomes larger. On the other hand, if a modulator acts on postsynaptic sites, pair-pulse depression should remain unchanged while current amplitude and/or decay time might be changed. Changes in decay time constant, as well as decreased current amplitudes and alterations in the P2:P1 ratio strongly suggest that both postsynaptic and presynaptic mechanisms contribute to the decreased synaptic strength of inhibitory transmission in the experimental group. In contrast, we found no significant difference in the synaptic strength (net charge transfer) of eEPSCs between the control ( $n=20$ cells) and experimental groups $(n=10$ cells, data not shown). 


\section{Discussion}

General anesthesia administered to 7-day-old rat pups causes long-lasting alterations in mitochondrial morphogenesis and regional distribution, heightened autophagocytic activity and ongoing neuropil destruction, as well as significant disturbances in synaptic neurotransmission in the subiculum.

Mitochondrial regeneration in neurons depends on balancing two opposing processes, mitochondrial fusion and fission. ${ }^{25}$ Deranged fusion leads to mitochondrial fragmentation; deranged fission leads to mitochondrial enlargement. Since our ultrastructural analyses indicate that general anesthesia causes significant enlargement of mitochondria, general anesthetics may modulate the fine equilibrium between fusion and fission. Disturbances in fusion and fission can lead to disturbances in mitochondrial functioning, particularly normal respiratory function. This may not be well tolerated by immature and functionally busy mammalian neurons, which, during development, are in need of adequate metabolic support. Indeed, impairment of mitochondrial morphogenesis may, at least in part, be the cause of reported anesthesiainduced developmental neurodegeneration, ${ }^{1,6,8,26-28}$, especially since an imbalance between fission and fusion appears to have a causal role in initiating several neurodegenerative diseases. ${ }^{29,30}$ Interestingly, large (and "giant") mitochondria are often described in aging neurons. ${ }^{31}$ Although it is tempting to draw a parallel between certain elements of neurodegeneration unique to aging and anesthesia-induced neurodegeneration unique to developmental brain it 
remains to be determined whether they share similar cellular pathways. In particular, it remains to be determined how anesthesia affects developmental fusion and fission. Although not within the scope of this study, future mechanistic studies using an easy-to-manipulate in-vitro system should focus on various GTPase proteins (e.g., Drp1, fis 1, OPA 1, mitofusin 1 and 2) that are crucial for proper pathway activation. ${ }^{32-34}$

It is possible that fusion and fission are modulated by general anesthesia and thus are the main causes of mitochondrial enlargement. However, our ultrastructural observations indicate that mitochondria are swollen and plagued by deranged, fragmented cristae and inner membranes. This suggests that the impairment of mitochondrial membrane integrity may be the main cause of their "leakiness," allowing the indiscriminate entry of colloids and water. In support of this notion is our previous finding that general anesthesia causes significant downregulation of bcl- $x_{\mathrm{L}}$ proteins, which are important in maintaining mitochondrial membrane integrity. This downregulation leads to significant cytochrome c leakage suggestive of increased mitochondrial permeability. ${ }^{1}$

Mitochondria have been classified by size in chronic neurodegenerative diseases such as Parkinson's and Alzheimer's, in which large mitochondria predominate, while the population of medium-sized mitochondria remains unchanged. ${ }^{35}$ We observed a similar tendency toward mitochondrial enlargement with a seemingly stable population of medium-sized mitochondria. Although medium-sized mitochondria may show a lower propensity for swelling 
than small ones, a more likely explanation is that the observed phenomenon is due to the shift in mitochondrial size distribution toward medium and large size category caused by the swelling of small and medium-sized mitochondria, respectively rather than a focal increase in mitochondria of any one size. Therefore, a detailed ultrastructural analysis of mitochondria should always accompany the size analysis.

Mitochondria are generated in the soma and move within the cytoplasm, which allows them to distribute within cells. ${ }^{36}$ Since neurons have multiple compartments (e.g., dendrites, axons, and synapses) that are located far from the cell body, they depend heavily on proper mitochondrial distribution. ${ }^{19}$ Since mitochondria are the main regulators of adenosine triphosphate (ATP) production, they are frequently found in the vicinity of active growth cones of developing neurons ${ }^{19}$ and in terminals with active synapses. ${ }^{20,21}$ We report that significantly fewer mitochondria are located in presynaptic neuronal profiles in anesthesia-treated subiculi than are in controls. Also, the mitochondrial profiles in presynaptic neuronal profiles are significantly larger than those in controls, suggesting that anesthesia-induced morphological changes shift the regional distribution of mitochondria away from very distant, thin, and highly arborized dendritic branches at a time when their presence is crucial for normal synapse formation and development. It remains to be determined whether general anesthesia impairs proper mitochondrial trafficking which may explain 
anesthesia-induced impairment of the morphogenesis and plasticity of dendritic spines and synapses. ${ }^{4,5}$

We report a decrease in mitochondrial density in both neuronal soma and presynaptic terminals which may suggest mitochondrial "dropout" due to mitochondrial degeneration and removal via autophagy. Having said that a decrease in mitochondrial density could be relative, caused by the fact that large mitochondria represent a bigger fraction of cytoplasmic and presynaptic terminal areas due to mitochondrial swelling or, perhaps, improper fission/fusion.

We show that the activity of the terminal enzyme of the respiratory chain, complex IV, is significantly up-regulated when measured 24 hours after anesthesia exposure, while the activity of complexes I and II/III remains unchanged. It is possible that an increase in complex IV activity provides anesthesia-treated neurons with increased ATP levels, ${ }^{37}$ which would result in a higher energy state in the neuron. Although it would be tempting to consider higher ATP levels to be beneficial for developing neurons, there is a drawback to the increased neuronal energy level based on an isolated increase in complex IV activity. Instead of leading to decreased oxidative stress by decreasing reactive oxygen species production, as previously thought, ${ }^{38}$ a recent report suggests that acute elevation of complex IV activity is associated with increased oxygen radical (ROS) production. ${ }^{23}$ In other words, a higher complex IV electron transfer rate onto oxygen, in view of intact activity of complexes I, II, and III, results in a higher degree of oxidation of the ubiquinone pool. This allows complexes II and III to 
transfer electrons to oxygen, causing an increase in ROS production. If general anesthesia leads to elevated ROS production, protein oxidation, and lipid peroxidation, this may, at least in part, explain the ongoing neuropil destruction and prominent neurite degradation. ${ }^{5}$

Here we focus on subiculum. It is noteworthy that the effects of general anesthesia on complex IV activity could be brain- region specific. For example, in a neurotoxic model of Parkinson's disease it has been shown that striatal, but not cortical neurons demonstrate elevated complex IV activity, resulting in ROS upregulation and neuronal cell death. ${ }^{37}$ Hence, it remains to be determined how general anesthesia affects the activity of complex IV and other ETC proteins in other brain regions that are vulnerable to anesthesia-induced developmental neurodegeneration.

We question whether our patch-clamp results showing that a single exposure to general anesthesia leads to lasting depression of inhibitory transmission in subicular neurons could be due to impaired regional distribution of mitochondria. This notion is based on the fact that defective synaptic transmission is associated with the loss of mitochondria from axon terminals. ${ }^{22}$ Interestingly, although inhibitory neurotransmission was impaired, excitatory neurotransmission was spared. Our earlier study using hippocampal slices of rats exposed to general anesthesia at age P7 demonstrated that excitatory synaptic transmission was not affected. ${ }^{8}$ This is intriguing considering that our morphometric studies of the subiculum show nonselective synapse loss ${ }^{5}$ and 
nonselective changes in mitochondrial regional distribution/ morphometry when excitatory and inhibitory synapses were examined (data not shown). The obvious reason for this selective functional synaptic plasticity is not known. However, it is of interest that functioning mitochondria and their ATP production are essential for maintaining normal synaptic physiology. ${ }^{34,39,40}$

Acute application of isoflurane or midazolam potentiates the inhibitory drive by heightening inhibitory synaptic activity mediated by gamma aminobutyric acid receptors. $^{41}$ In contrast, nitrous oxide silences excitatory (N-methylD-aspartate-mediated) synaptic transmission. ${ }^{42}$ Thus, it is possible that, as a consequence of an inadequate metabolic ratio of supply to demand, anesthesiainduced degenerative changes in mitochondria and a potential decrease in ATP production preferentially impair highly activated inhibitory synaptic function. Other studies have indicated that free radical signaling may be important in modulating synaptic transmission. For example, acute applications of hydrogen peroxide, the common donor of ROS, preferentially reduced inhibitory over excitatory synaptic transmission in thalamocortical, ${ }^{43}$ hippocampal, ${ }^{44,45}$ cortical, and striatal slices ${ }^{45}$ by both pre- and post-synaptic mechanisms. Since the strength of elPSCs but not eEPSCs in subiculi is greatly diminished in slices of rats exposed to clinical anesthesia early in life, it is tempting to speculate that this can be at least in part a result of the production of ROS in response to anesthetic-induced mitochondrial dysfunction. However, the precise mechanism for the selective homeostatic changes in neuronal function under extensive 
GABAergic stimulation associated with various physiological and pathological conditions remains to be examined. ${ }^{46}$

Because damaged mitochondria could become an uncontrollable source of free oxygen radicals and therefore would have to be degraded to ensure neuronal survival, it came as no surprise that general anesthesia created a substantial amount of biological "garbage" and heightened autophagy. Autophagy, a multistep process, is initiated by the formation of autophagosomes, double-membrane-bound cellular structures that enter lysosomes, acidic vacuolar compartments containing various lytic enzymes that have $\mathrm{pH}$ optima in the acidic range. ${ }^{47-49}$ Lysosomes may slowly leak enzymes, which, in turn, can induce apoptosis via activation of a variety of pro-caspases. Our observation of a substantial number of autophagic bodies, in addition to impaired mitochondrial morphogenesis, neuropil damage, and synapse loss $^{5}$ raises the important possibility that general anesthesia kills developing neurons simply by overwhelming natural autophagy with a massive production of defective mitochondria. Further studies will be necessary to test this possibility.

Although a reliable model for studying developmental neurodegeneration our anesthesia protocol is based on the use of anesthetics in combination. As such it prevents us from deciphering the relative contribution of each agent. Further studies focused on individual anesthetics will help us decipher their relative importance in inducing mitochondrial morphological impairments and dysfunction. 
We show that general anesthesia causes significant impairment in mitochondrial morphogenesis and their regional distribution in developing subiculi of rat pups. Hence, mitochondria may be the most vulnerable initial target of anesthesia-induced developmental neurotoxicity. 


\section{References}

1. Yon J-H, Daniel-Johnson J, Carter LB, Jevtovic-Todorovic V: Anesthesia induces neuronal cell death in the developing rat brain via the intrinsic and extrinsic apoptotic pathways. Neuroscience 2005; 35:815-27

2. Wilder RT, Flick RP, Sprung J, Katusic SK, Barbaresi WJ, Mickelson C, Gleich SJ, Schroeder DR, Weaver AL, Warner DO: Early exposure to anesthesia and learning disabilities in a population-based birth cohort. Anesthesiology 2009; 110:796-804

3. Yon J-H, Carter LB, Reiter RJ, Jevtovic-Todorovic V: Melatonin reduces the severity of anesthesia-induced apoptotic neurodegeneration in the developing rat brain. Neurobiol Dis. 2006; 21: 522-30

4. Head BP, Patel HH, Niesman IR, Drummond JC, Roth DM, Patel PM: Inhibition of p75 neurotrophin receptor attenuates isoflurane-mediated neuronal apoptosis in the neonatal central nervous system. Anesthesiology $2009 ; 110: 813-25$

5. Lunardi N, Ori C, Erisir A, Jevtovic-Todorovic V: General anesthesia causes long-lasting disturbances in the ultrastructural properties of developing synapses in young rats. Neurotox Res. 2010; 17:179-88

6. Lu LX, Yon J-H, Carter LB, Jevtovic-Todorovic V: General anesthesia activates BDNF-dependent neuroapoptosis in the developing rat brain. Apoptosis 2006; 11:1603-15 
7. Jevtovic-Todorovic V, Benshoff N, Olney JW: Ketamine potentiates cerebrocortical damage induced by the common anaesthetic agent nitrous oxide in adult rats. Br J Pharmacol. 2000; 130:1692-8

8. Jevtovic-Todorovic V, Hartman RE, Izumi Y, Benshoff ND, Dikranian K, Zorumski CF, Olney JW, Wozniak DF: Early exposure to common anesthetic agents causes widespread neurodegeneration in the developing rat brain and persistent learning deficits. J. Neurosci. 2003; 23: 876-82

9. Paxinos G, Watson C: The rat brain in stereotaxic coordinates. Sydney: Academic. 1986

10. Erisir A, Harris JL: Decline of the critical period of visual plasticity is concurrent with the reduction of NR2B subunit of the synaptic NMDA receptor in layer 4" J Neurosci 2003; 23:5208-18

11. Crain B, Cotman C, Taylor D, Lynch G: A quantitative electron microscopic study of synaptogenesis in the dentate gyrus of the rat. Brain Res. 1973; 63:195-204

12. Pérez-Carreras M, Del Hoyo P, Martín MA, Rubio JC, Martín A, Castellano G, Colina F, Arenas J, Solis-Herruzo JA: Defective hepatic mitochondrial respiratory chain in patients with nonalcoholic steatohepatitis. Hepatology 2003; 38:999-1007

13. Joksovic PM, Weiergraber M, Lee WY, Struck H, Schneider T, Todorovic SM: Isoflurane-sensitive presynaptic R-type calcium channels contribute to inhibitory synaptic transmission in the rat thalamus. J Neurosci 2009; 
14. Stevens WC, Kingston HGG: Inhalation anesthesia. In Clinical Anesthesia, $2^{\text {nd }}$ ed. Barash PG, J.B. Lippincott, Philadelphia, 1992, pp. 429-65

15. Mannella CA: The relevance of mitochondrial membrane topology to mitochondrial function. Biochim Biophys Acta 2006; 1762:140-7

16. Rizzi S, Carter LB, Ori C, Jevtovic-Todorovic V: Clinical anesthesia causes permanent damage to the fetal guinea pig brain. Brain Pathology 2008; $18: 198-210$

17. Nikizad H, Yon J-H, Carter LB, Jevtovic-Todorovic V: Early exposure to general anesthesia causes significant neuronal deletion in the developing rat brain. Ann NY Acad Sci 2007; 1122:69-82

18. McNaughton $\mathrm{N}$ : The role of the subiculum within the behavioural inhibition system. Behav Brain Res 2006; 174:232-50

19. Morris RL, Hollenbeck PJ: The regulation of bidirectional mitochondrial transport is coordinated with axonal outgrowth. J. Cell Sci. 1993; 104: 917-27

20. Rowland KC, Irby NK, Spirou GA: Specialized synapse-associated structures within the calyx of Held. J. Neurosci. 2000; 20: 9135-44

21. Shepherd GM, Harris KM: Three-dimensional structure and composition of $\mathrm{CA} 3 \rightarrow$ CA1 axons in rat hippocampal slices: Implications for presynaptic connectivity and compartmentalization. J. Neurosci. 1998; 18: 8300-10

22. Stowers RS, Megeath LJ, Gorska-Andrzejak J, Meinertz IA, Schwarz TL: Axonal transport of mitochondria to synapses depends on Milton, a novel Drosophila protein. Neuron 2002; 36: 1063-77 
23. Dröse $\mathrm{S}$ and Brandt U: The mechanism of mitochondrial superoxide production by cytochrome bc1 complex. J. Biol. Chem. 2008; 283: 21649-54

24. Zucker RS, Regehr WG: Short-term synaptic plasticity. Ann. Rev Physiol. 2002; 64: 355-405

25. Chan DC: Mitochondrial fusion and fission in mammals. Annu Rev Cell Dev Biol. 2006; 22:79-99

26. Ikonomidou C, Bosch F, Miksa M, Bittigav P, Vockler J, Dikranian K, Tenkova TI, Stefovska V, Turski L, Olney JW: Blockade of NMDA receptors and apoptotic neurodegeneration in the developing brain. Science 1999; 283: 70-4

27. Ikonomidou C, Bittigau P, Ishimaru MJ, Wozniak DF, Kock C, Genz K, Price MT, Stefovska V, Horster F, Tenkova T, Dikranian K, Olney JW: Ethanolinduced apoptotic neurodegeneration and fetal alcohol syndrome. Science 2000; 287: 1056-60

28. Slikker W. Jr, Zou X, Hotchkiss CE, Divine RL, Sadovova N, Twaddle NC, Doerge DR, Scallet AC, Patterson TA, Hanig JP, Paule MG, Wang C: Ketamine-induced neuronal cell death in the perinatal rhesus monkey. Toxicol Sci 2007; 98: 145-58

29. Bossy-Wetzel E, Barsoum MJ, Godzik A, Schwarzenbacher R, Lipton SA: Mitochondrial fission in apoptosis, neurodegeneration and aging. Curr Opin Cell Biol 2003; 15: 706-16 
30. Wang X, Su B, Lee HG, Li X, Perry G, Smith MA, Zhu X: Impaired balance of mitochondrial fission and fusion in Alzheimer's disease. J Neurosci. 2009; 29:9090-103

31. Navarro A, Boveris A: Brain mitochondrial dysfunction in aging, neurodegeneration, and Parkinson's disease. Front Aging Neurosci. 2010; 2: pii:34.

32. Smirnova E, Griparic L, Shurland DL, van der Bliek AM: Dynamin-related protein Drp1 is required for mitochondrial division in mammalian cells. Mol Biol Cell 2001; 12: 2245-56

33. Zorzano A, Liesa M, Sebastián D, Segalés J, Palacín M: Mitochondrial fusion proteins: Dual regulators of morphology and metabolism. Semin Cell Dev Biol 2010; $21: 566-74$

34. Li Z, Okamoto K, Hayashi Y, Sheng M: The importance of dendritic mitochondria in the morphogenesis and plasticity of spines and synapses. Cell 2004; 119:873-87

35. Trimmer PA, Swerdlow RH, Parks JK, Keeney P, Bennett JP Jr, Miller SW, Davis RE, Parker WD Jr: Abnormal mitochondrial morphology in sporadic Parkinson's and Alzheimer's disease cybrid cell lines. Exp Neurol. 2000; 162: $37-50$

36. Yaffe MP: The machinery of mitochondrial inheritance and behavior. Science 1999; 283:1493-7 
37. Singh S, Misiak M, Beyer C, Arnold S: Brain region specificity of 3nitropropionic acid-induced vulnerability of neurons involves cytochrome c oxidase. Neurochem Int. 2010; 57: 297-305

38. Cadenas E, Boveris A, Ragan $\mathrm{Cl}$ and Stoppani AO: Production of superoxide radicals and hydrogen peroxide by $\mathrm{NADH}$-ubiquinone reductase and ubiquinol-cytochrome c reductase from beef-heart mitochondria. Arch.

Biochem. Biophys. 1977; 180: 248-57

39. Inquimbert P, Rodeau JL, Schlichter R: Regional differences in the decay kinetics of $\operatorname{GABA}(\mathrm{A})$ receptor-mediated miniature IPSCs in the dorsal horn of the rat spinal cord are determined by mitochondrial transport of cholesterol. J Neurosci 2008; 28:3427-37

40. Jonas E: Regulation of synaptic transmission by mitochondrial ion channels. J Bioenerg Biomembr. 2004; 36:357-61

41. Franks NP: General anaesthesia: From molecular targets to neuronal pathways of sleep and arousal. Nat Rev Neurosci 2008; 9:370-86

42. Mennerick S, Jevtovic-Todorovic V, Todorovic SM, Shen W, Olney JW, Zorumski CF: Effect of nitrous oxide on excitatory and inhibitory synaptic transmission in hippocampal cultures. J Neurosci 1998; 18:9716-26

43. Frantseva MV, Perez Velazquez JL, Carlen PL: Changes in membrane and synaptic properties of thalamocortical circuitry caused by hydrogen peroxide. J Neurophysiol. 1998; 80: 1317-26 
44. Müller M, Fontana A, Zbinden G, Gähwiler BH: Effects of interferons and hydrogen peroxide on CA3 pyramidal cells in rat hippocampal slice cultures. Brain Res. 1993; 619: 157-62

45. Sah R, Galeffi F, Ahrens R, Jordan G, Schwartz-Bloom RD: Modulation of the GABA(A)-gated chloride channel by reactive oxygen species. J Neurochem. 2002; 80: 383-91

46. Mody I: Aspects of the homeostatic plasticity of GABAA receptor-mediated inhibition. J Physiol. 2005; 562:37-46

47. Brunk UT, Terman A: The mitochondrial-lysosomal axis theory of aging: Accumulation of damaged mitochondria as a result of imperfect autophagocytosis. Eur J Biochem. 2002; 269:1996-2002

48. Terman A, Gustafsson B, Brunk UT: Autophagy, organelles and ageing. J. Pathol. 2007; 211:134-43

49. Levine B, Yuan J: Autophagy in cell death: An innocent convict? J Clin Invest. 2005; 115:2679-88 


\section{Figure Legends:}

Figure 1: Anesthesia causes long-lasting ultrastructural changes in mitochondria in subiculi of 21-day-old rats. $A, C)$ The pyramidal neuron (A) and neuropil (C; synaptic contacts are noted with arrows) in a subiculum from a control rat show abundant small mitochondria with no evidence of swelling or injury. $B, D)$ Mitochondria in the perikarion of a pyramidal neuron (B) and nerve terminals in a neuropil (D) of subiculum from experimental rats display structural disorganization of cristae (asterisks), as well as dilated intracristal spaces with vacuoles and overall swelling. Note the presence of dark, condensed mitochondria in late stages of degeneration (arrows) (magnification 12,000x). N nucleus.

Figure 2: Anesthesia promotes autophagic activity, as shown in subicular pyramidal neurons of 21-day-old rats. A) In experimental pyramidal neurons, numerous lysosomes (double asterisks) and autophagic vacuoles (arrowheads) were dispersed throughout the cytoplasm. B) Autophagosomes, double-layered membrane structures, were frequently noted in experimental neurons where parts of cannibalized mitochondria could be detected (single asterisk). 
Figure 3: Morphometric analysis of mitochondria in the perikaryon of pyramidal subicular neurons of 21-day-old rats. A) Mitochondria in the experimental neurons occupy significantly more cytoplasmic soma than do those in controls $\left(22.5 \%\right.$ vs. $\left.13.44 \%,{ }^{*} p<0.05\right)(n=15$ neurons per group from 3 animals each). B) In experiental animals, mitochondrial density, presented as the number of mitochondria per unit area $\left(\mu \mathrm{m}^{2}\right)$ of cytoplasmic soma, is significantly lower in pyramidal neurons than that in controls $\left({ }^{*}, p<0.05, n=15\right.$ neurons per group from 3 control and 3 experimental pups; control and experimental pups were litter-matched).

Figure 4: Mitochondrial size classification in the perikaryon of pyramidal subicular neurons of 21-day-old rats. A) A small fraction of mitochondria (up to $0.05 \mu \mathrm{m}^{2}$ ) constitutes about $15 \%$ of total mitochondria in control pyramidal neurons, but only $5 \%$ in the experimental pyramidal neurons $\left.\left({ }^{* * *}, p<0.001\right) . B\right)$ A medium-sized fraction of mitochondria $\left(0.06\right.$ to $\left.0.25 \mu \mathrm{m}^{2}\right)$ represents the largest population of mitochondria in subicular pyramidal neurons. This fraction remains unchanged after anesthesia treatment. C) A large fraction of mitochondria $\left(0.26\right.$ to $\left.0.65 \mu \mathrm{m}^{2}\right)$ constitutes only about $5 \%$ of total mitochondria in control pyramidal neurons, but $15 \%$ of those in experimental pyramidal neurons $\left({ }^{*}, p<0.05\right)$. D) An extra-large fraction of mitochondria (more than 0.65 $\mu \mathrm{m}^{2}$ ) represents less than $1 \%$ of the total number of mitochondria in 
experimental pyramidal neurons and shows over two-fold higher prevalence in these neurons (Fig. 3D) although it did not reach statistical significance $(n=15$ neurons per group from 3 control and 3 experimental pups; control and experimental pups were litter-matched).

Figure 5: Anesthesia decreases mitochondrial density in presynaptic neuronal terminal subicular neuropils of 21-day-old rats. Compared to controls, fewer presynaptic neuronal terminals in experimental subicular neuropils contain mitochondrial profiles. When the findings are presented as a percentage of presynaptic profiles containing mitochondria, a significantly (about two-fold) higher percentage of mitochondria-containing presynaptic profiles occurs in control subiculi than in experimental subiculi $\left({ }^{*}, p<0.05\right)(n=28$ photo frames/group from 4 control and 4 experimental pups from two different litters; control and experimental pups were litter-matched).

Figure 6: Morphometric analysis of mitochondria in presynaptic neuronal terminals in subicular neuropils of 21-day-old rats. A) Morphometric analysis of the area of mitochondrial profiles shows that experimental mitochondrial profiles were approximately $30 \%$ larger than that of controls $\left.\left({ }^{*}, p<0.05\right)\right)(n=30$ photo frames obtained from 5 control pups; $n=45$ photo frames obtained from 5 experimental pups; control and experimental pups were litter-matched; total of three different litters were used). B) There is no difference between control and experimental subiculi with regard to the areas of mitochondria-containing 
presynaptic nerve terminals $(n=30$ photo frames obtained from 5 control pups; $n$ $=45$ photo frames obtained from 5 experimental pups; control and experimental pups were litter-matched; total of three different litters were used). C) The calculated mitochondrial index (ratio between mitochondrial area and mitochondria-containing presynaptic area) was significantly higher in experimental subicular neuropils $\left({ }^{*}, p<0.05\right)$ than in control neuropils.

Figure 7: Anesthesia differentially modulates the activity of mitochondrial respiratory chain proteins. A) Compared to control subiculi, the activity of complex IV in experimental subiculi is significantly increased at $24 \mathrm{~h}$ after anesthesia $\left({ }^{*} p<0.05\right)(n=8$ pups in control group; $n=6$ pups in experimental group). B) The activity of complex I was unchanged in the anesthesia-treated group as compared to sham controls ( $n=5$ pups per group). C) The activity of complex II was unchanged in the anesthesia-treated group as compared to sham controls ( $n=3$ pups in control group; $n=4$ pups in experimental group). The activity of complexes I, II, and IV were expressed as ratios [per the activity of citrate synthase (CS)] since citrate synthase activity is directly proportional to mitochondrial content.

Figure 8: Alterations occurred in inhibitory synaptic transmission in pyramidal cells of subiculi after exposure to anesthesia early in life. 
A) Representative elPSCs obtained using a paired-pulse protocol to record from two pyramidal cells in the subiculi of rats in the control (black trace) and experimental groups (gray trace). Note that the experimental group had decreased current amplitude and faster decay. Arrows indicate the time of paired-pulse stimulus application (interval $1.1 \mathrm{sec}$ ). Stimulus transients have been removed for clarity of the current traces. B) Histograms showing average data from control cells $(n=14)$ and experimental cells $(n=12)$. Black solid bars indicate control cells; gray bars represent experimental cells; vertical lines indicate the SEM of multiple determinations. All data are normalized to $100 \%$ of average responses in the control group. Left panel shows a decrease in net charge transfer of elPSCs from $100 \pm 18 \%$ to $51 \pm 9 \%(p<0.05)$ in the experimental group; middle panels show a decrease in decay of tau from $100 \pm$ $16 \%$ to $59 \pm 7 \%$; right panels show a small but significant increase in P2/P1 from $100 \pm 2 \%$ to $107 \pm 1 \%(p<0.05)$. 
Fig. 1

\section{Sham controls \\ SOMA \\ Anesthesia-treated}

A
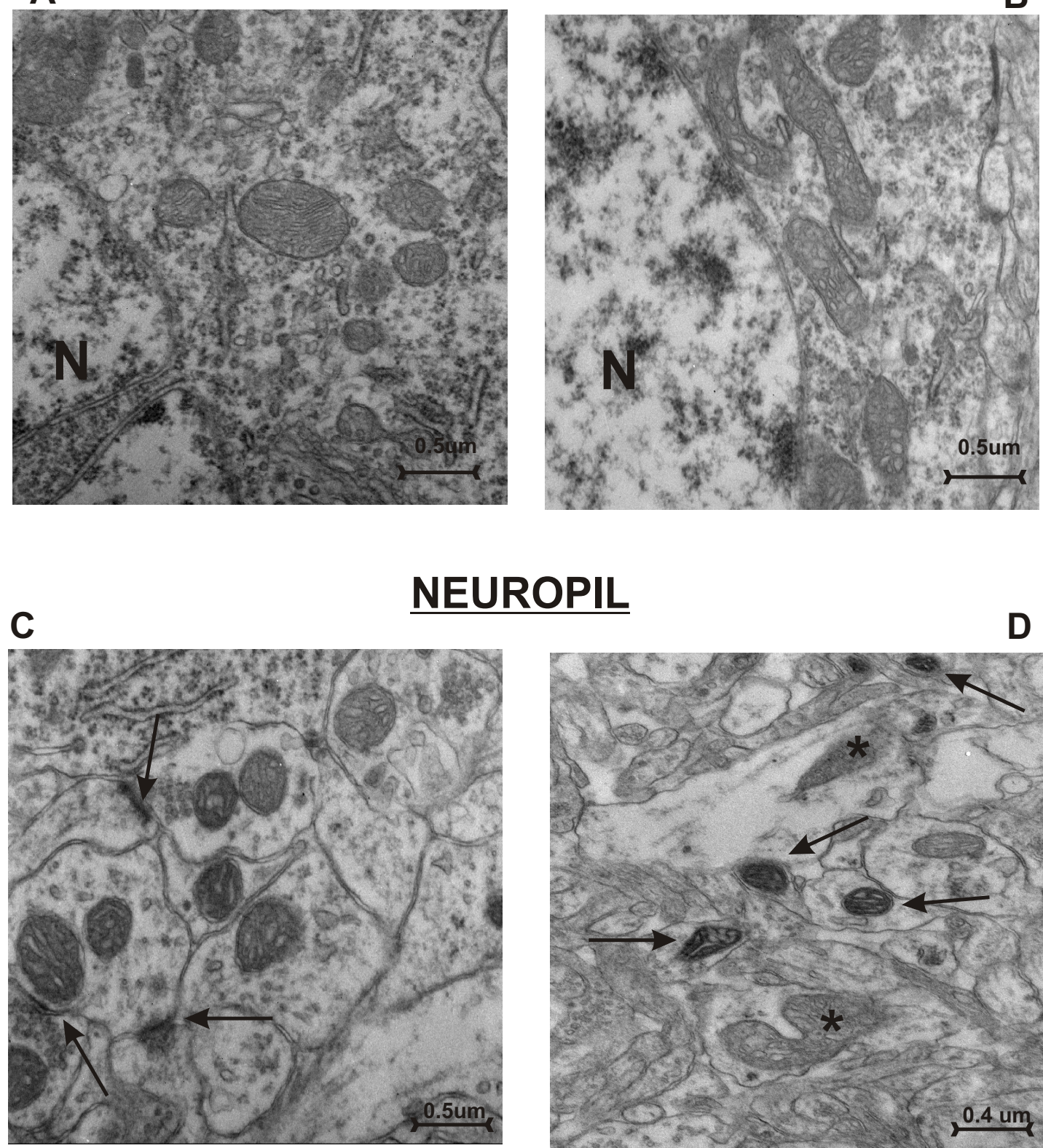

D

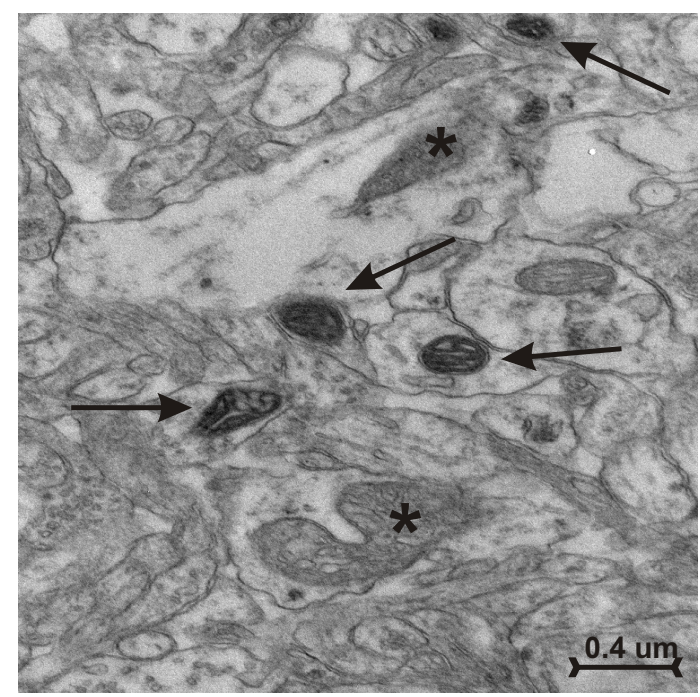


A

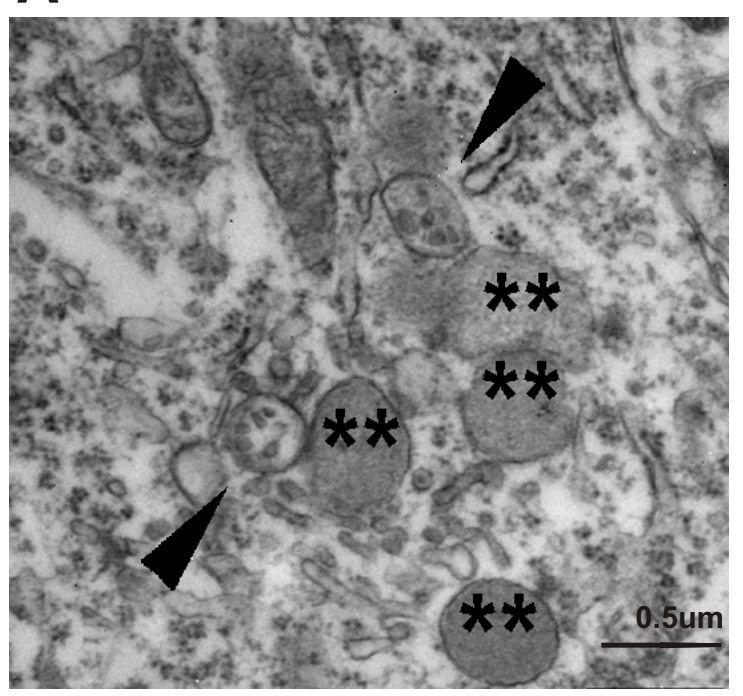

Fig. 2

B

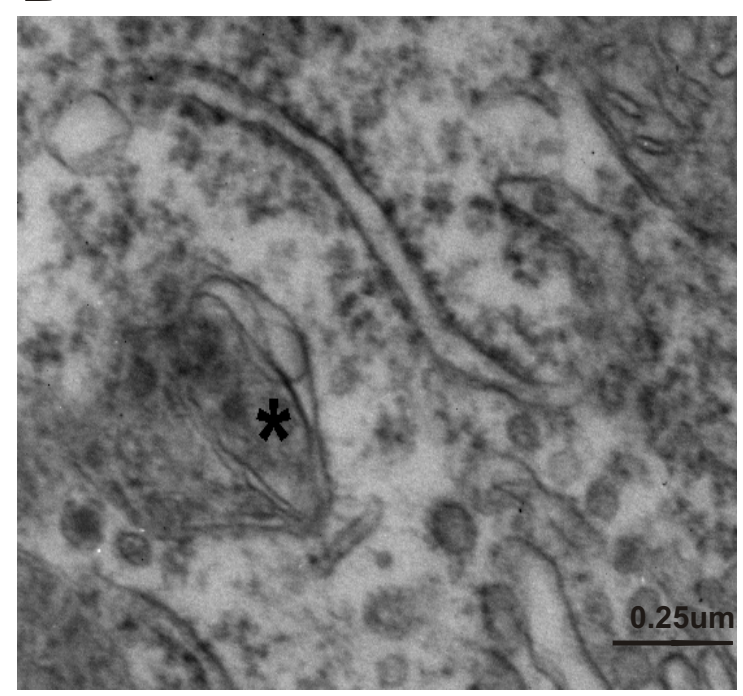

Fig. 3

A

Mitochondrial area

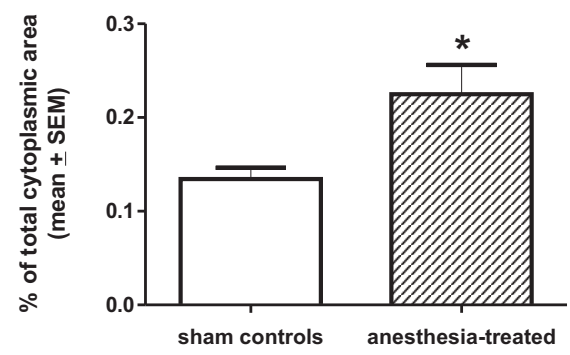

B

Mitochondrial density

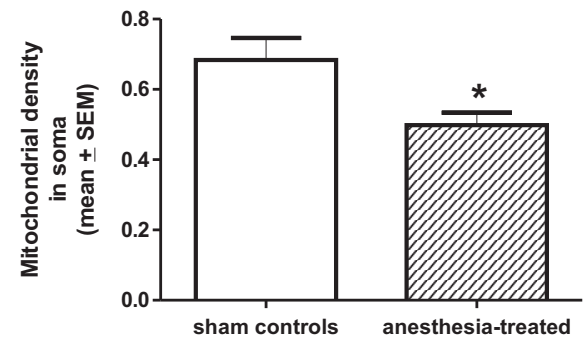


Fig. 4

A

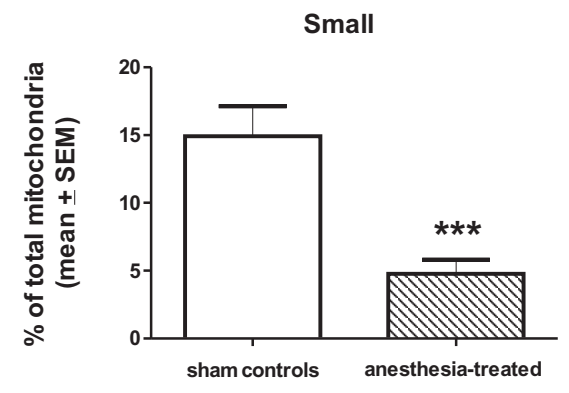

C

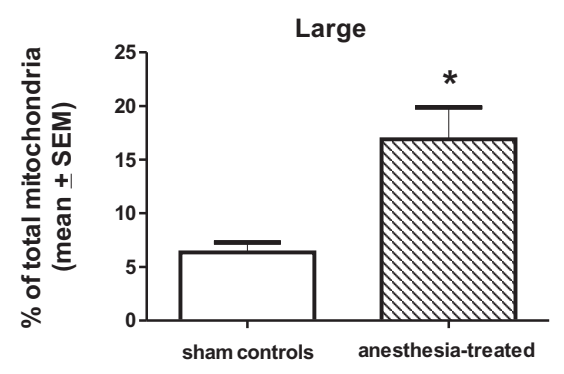

B

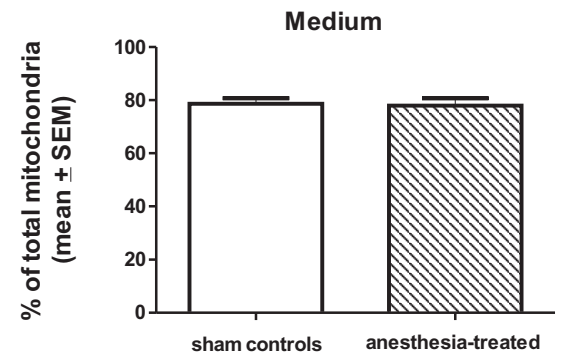

D

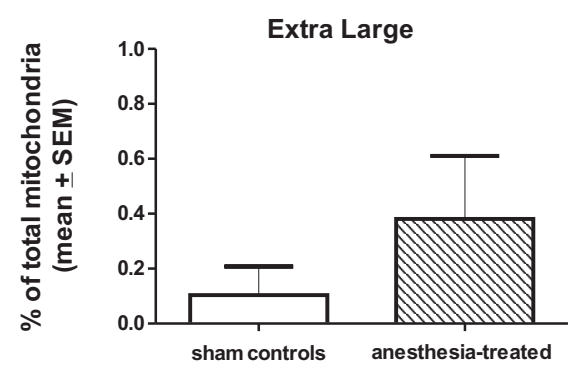


Fig. 5

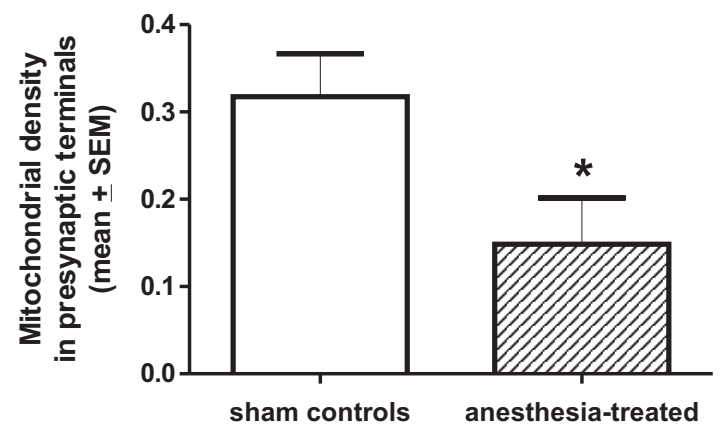




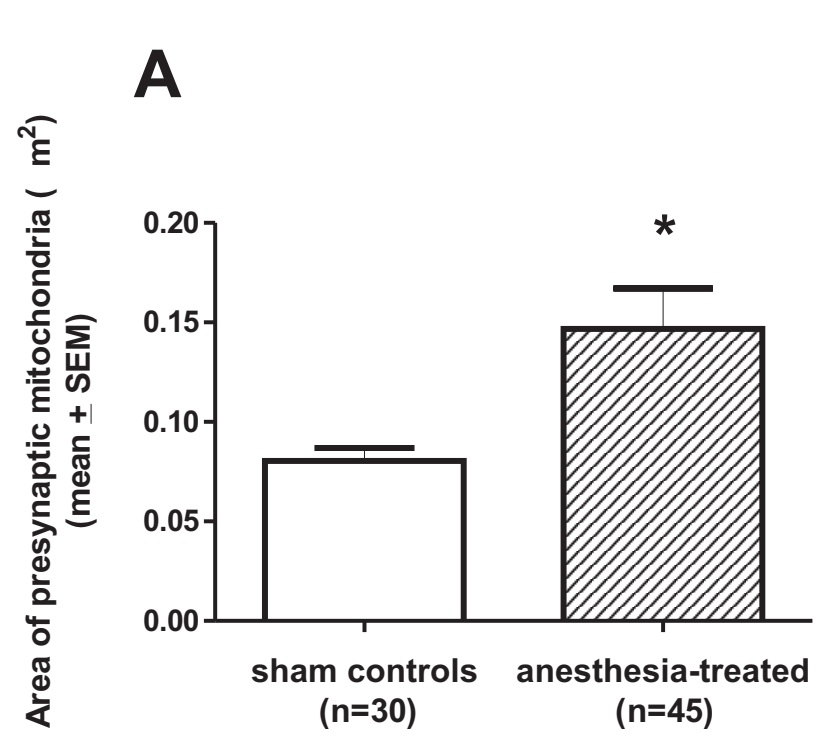

Fig. 6
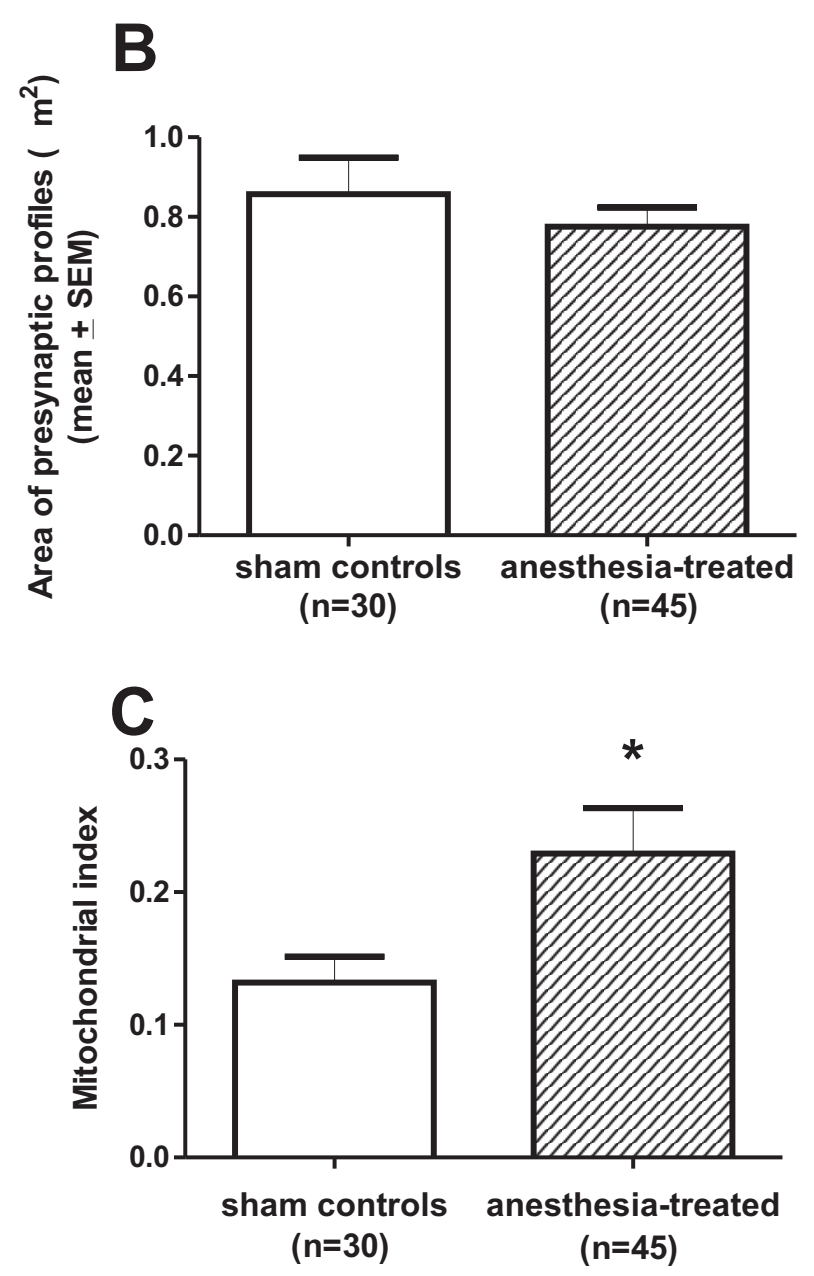


\section{A Complex IV}

Fig. 7

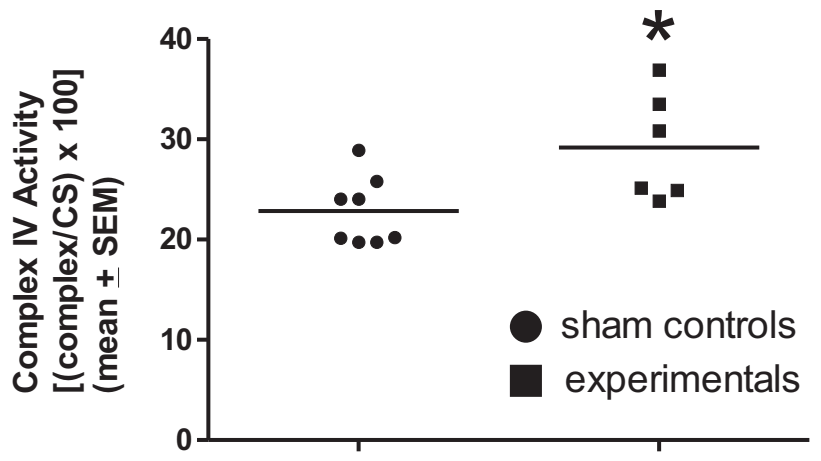

B Complex I

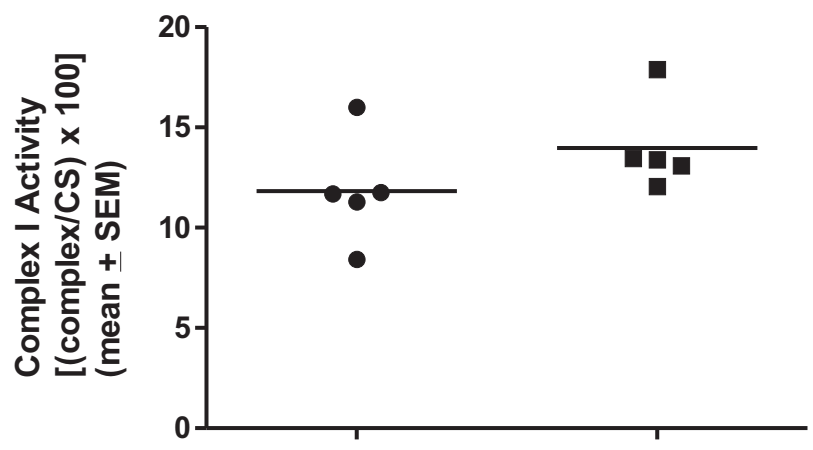

C Complex II/III

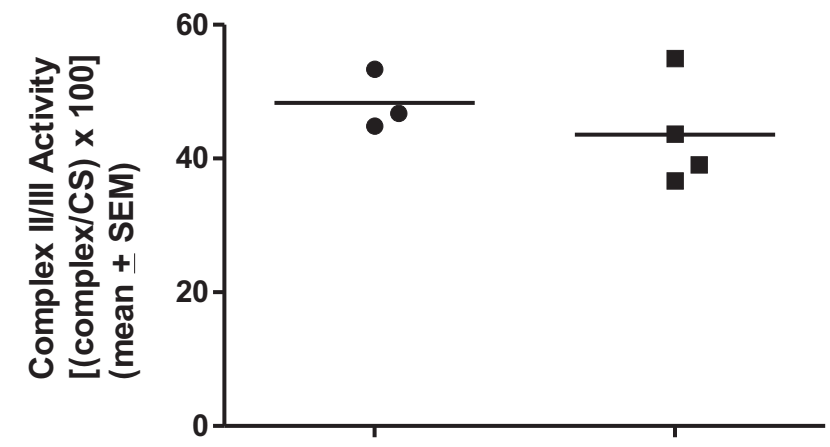


Figure 8

A

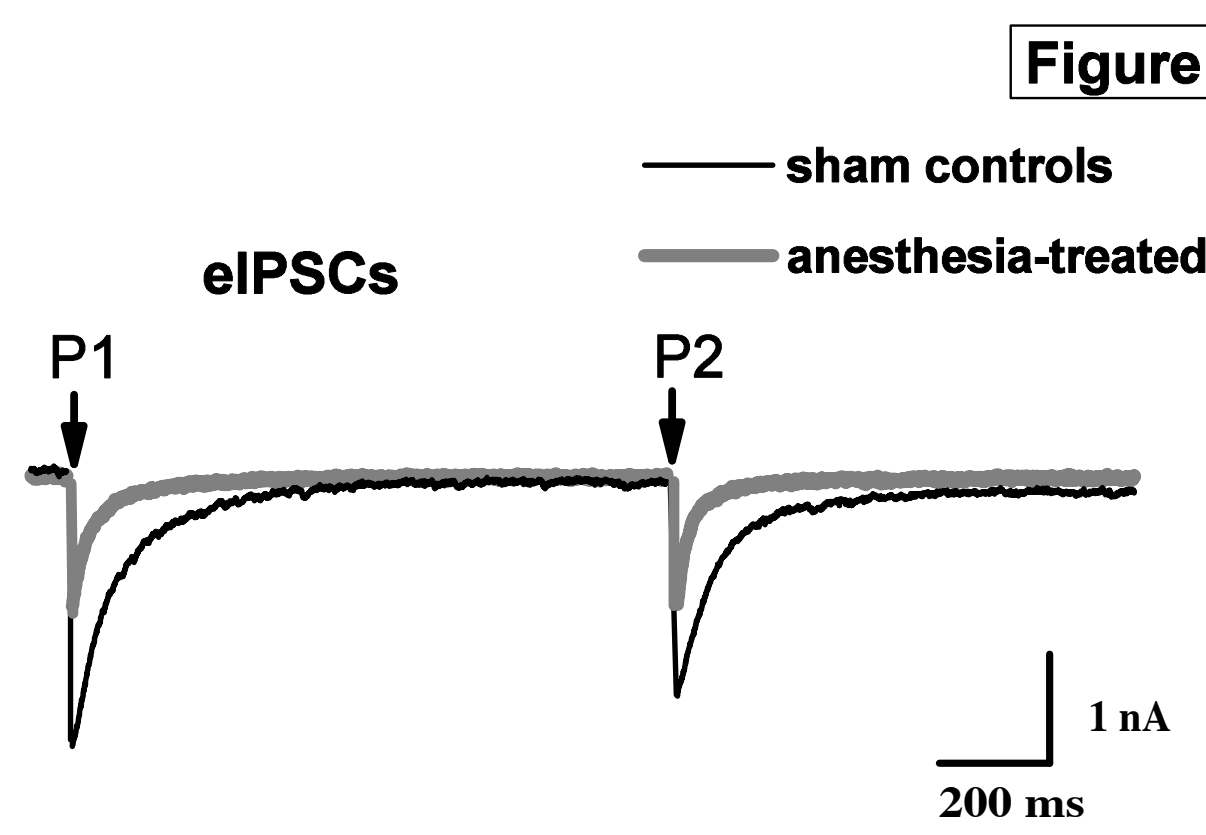

B

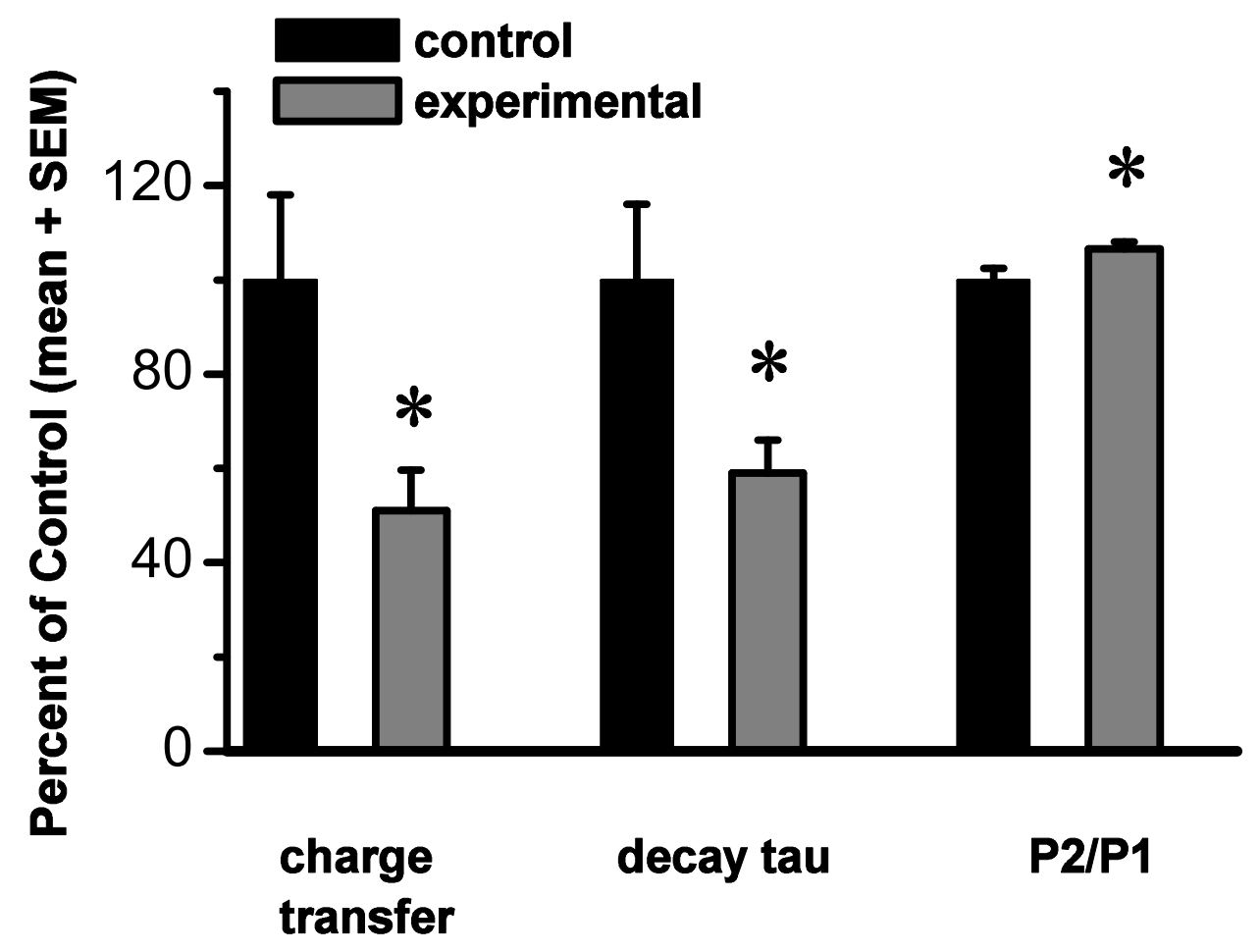

